\title{
SEMI-AUTOMATED LINKING OF \\ USER INTERFACE DESIGN ARTIFACTS
}

by

Said Selim Elnaffar

\begin{abstract}
I thesis submitted to the Department of
Computer and Information Science in conformity

with the requirements for the degree of
\end{abstract}

Master of Science

Queen's University

Kingston, (Ontario, Canada

May, 1999

Copyright (C) Said Elnaffar, 1999 
National Library of Canada

Acquisitions and Bibliographic Services

395 Wellington Street Ottawa ON K1A ON4 Canada
Bibliothèque nationale

du Canada

Acquisitions et services bibliographiques

395, rue Wellington

Ottawa ON KIA ON4

Canada
The author has granted a nonexclusive licence allowing the National Library of Canada to reproduce, loan, distribute or sell copies of this thesis in microform, paper or electronic formats.
L'auteur a accordé une licence non exclusive permettant à la Bibliothèque nationale du Canada de reproduire, prêter, distribuer ou vendre des copies de cette thèse sous la forme de microfiche/film, de reproduction sur papier ou sur format électronique.

L'auteur conserve la propriété du droit d'auteur qui protège cette thèse. $\mathrm{Ni}$ la thèse ni des extraits substantiels de celle-ci ne doivent être imprimés ou autrement reproduits sans son autorisation.
The author retains ownership of the copyright in this thesis. Neither the thesis nor substantial extracts from it may be printed or otherwise reproduced without the author's permission.

$0-612-42610-6$ 


\begin{abstract}
User centered design involves the creation of design artifacts such as task and architecture models. It is increasingly accepted that such artifacts cannot effectively be created separately, but instead coevolve incrementally, so that information obtained from the development of one artifact contributes to the development of the others. In user interface development, different people with different backgrounds rypically participate in the development of these artitacts. Consequently, communication is important for coevolution. This research demonstrates how different design ardiacts can be linked semi-automatocally. We illustrate this technique using - Ldligo, a computer-based tool for generating links between the User Action Noration (L'. IN) task model and the Clock archirecrural model. ()ur results show that in rwo case studies, we were able to generate $900^{\circ}$ " of possible links with error rate of 0$)^{\circ}$ " to $12^{\prime \prime} "$ with limited human assistance.
\end{abstract}




\section{ACKNOWLEDGEMENTS}

I wish to express sincere appreciation to:

Nick Graham, who has been and remains a real friend, an outstanding teacher and a brilliant young supervisor.

My mom, who prays for me all the rime...

Niveen, my wife, for her unique inspiration and her unforgettable delicious meals...

My daughters, Asalah and Sarah who were alway's reminding me to take a break and watch our favorite 'TV shows. Artbur, Barmey and Teletubbiest

A long list of friends that is not limited to: Gary Anderson, James Cordy, ()mar Flsousi, Ihab Elshoubaki, Greg Phillips, Laurie Ricker, Hugh Stewart, Tore Urnes, Tim Wright and others, too numerous to mention here... but they are all in my heart.

Finully, I am grateful to my beloved Canada... to her soil... to her winter and summer... to her people...to everything belonging to this land -- because everything about her is just sweet...! 


\section{TABLE OF CONTENTS}

ABSTRACT

ACKNOWLEDGEMENTS .......................................................................................................................ii

TABLE OF CONTENTS ..................................................................................................................iii

LIST OF TABLES

LIST OF FIGURES .................................................................................................................

1. INTRODUCTION.....................................................................................................................................

1.1 PROBLEM AND MOTIVATION …….....................................................................................................

1.2 THE PURPOSE OF THIS THESIS........................................................................................................

1.3 OUR APPROACH TO SOLVING THIS PROBLEM: ADLIGO METHODOLOGY ........................6

1.4 RESULTS AND ANALYSIS...............................................................................................................

1.5 CONTRIBUTIONS .....................................................................................................................................

1.6 ROAD MAP .............................................................................................................................. 


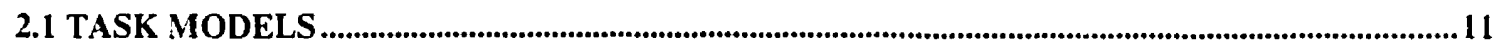

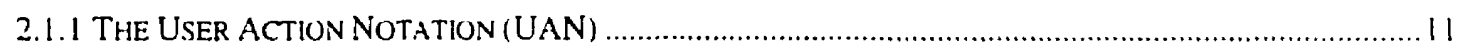

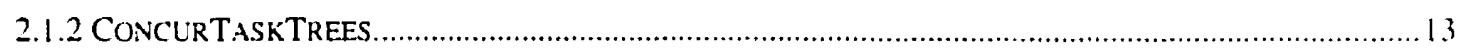

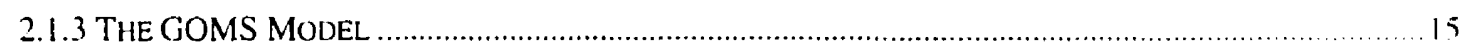

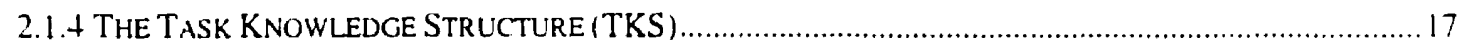

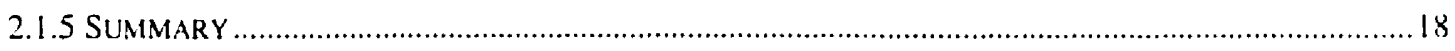

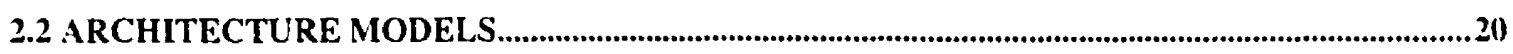

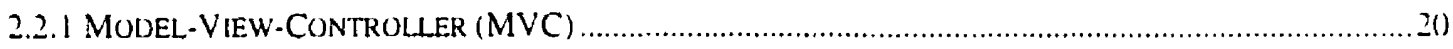

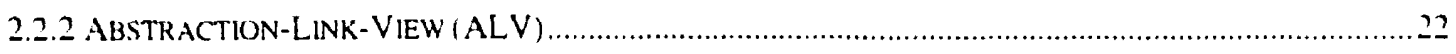

2.2 .3 CLOCK

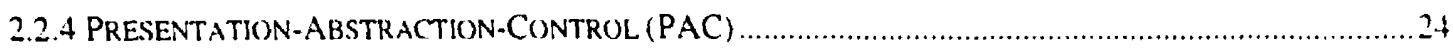

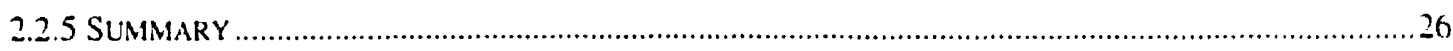

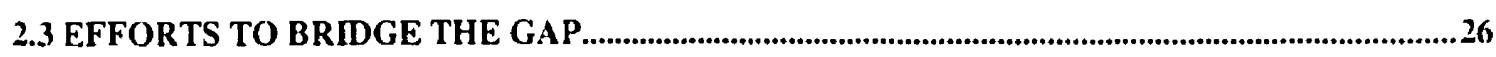

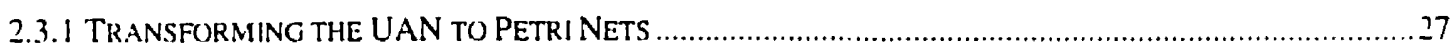

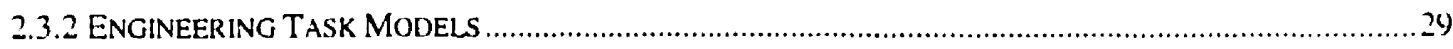

2.3 .3 ADEPT

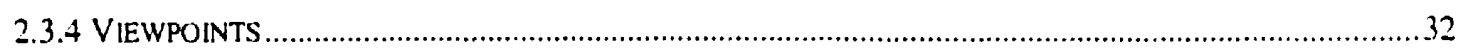

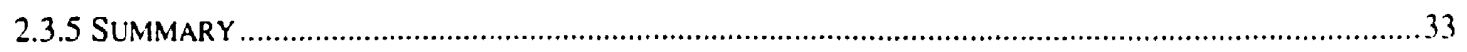


3.1 TASK MODELING: TASK HIERARCHY FOR PROJECT PLANNING.........................................37

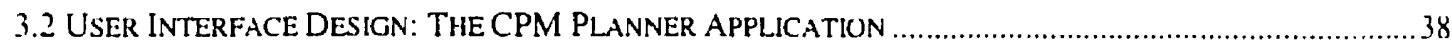

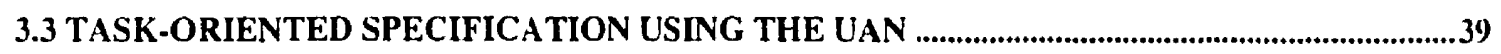

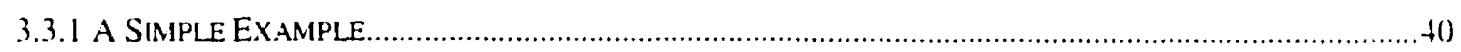

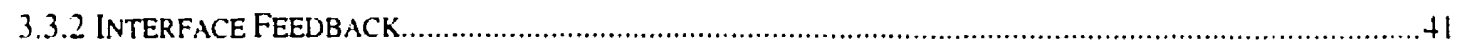

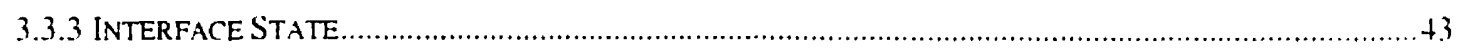

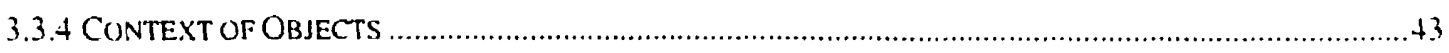

3.3.5 SUMMARY

3.4 BUILDING THE CPM PLANNER USING THE CLOCK ARCHITECTURE STYLE...................4

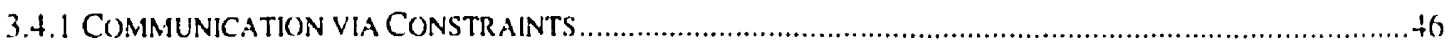

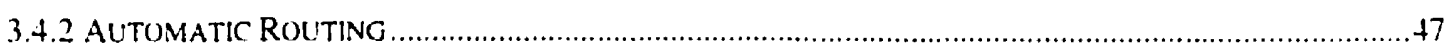

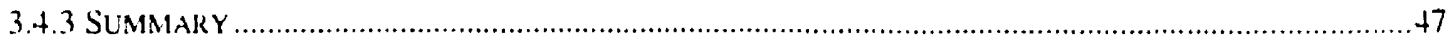

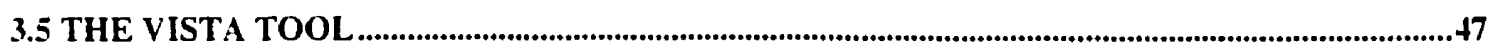

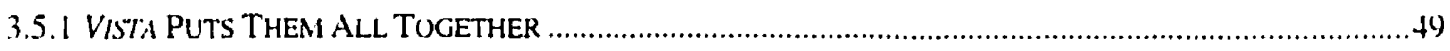

3.5.2 LINKING THE UAN CONTEXTS TO ARCHITECTURE COMPONENTS .................................................49

3.5.3 LINKING THE UAN ACTIONS TO ARCHITECTURE COMPONENTS...................................................

3.5.4 LINKING THE UAN INTERFACE FEEDBACK SPECIFICATIONS TO COMPONENT VIEWS ........................52

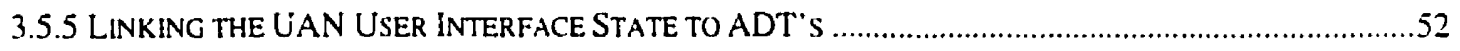

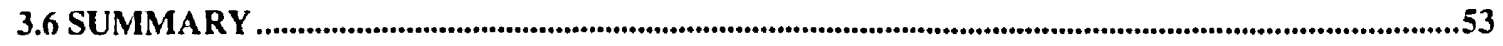


4.1 THE FRAMEWORK OF THE LINK GENERATION PROCESS ....................................................56

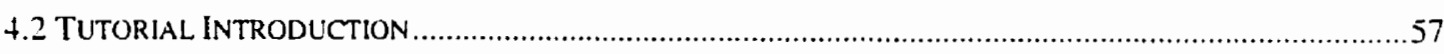

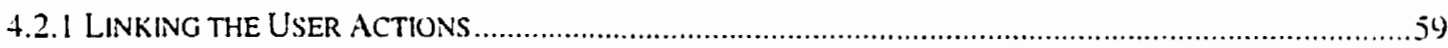

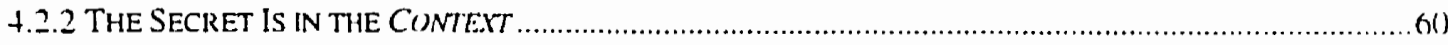

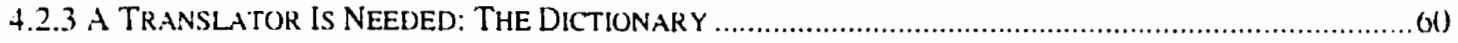

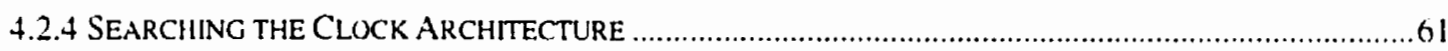

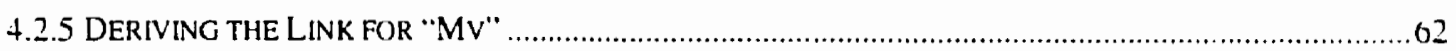

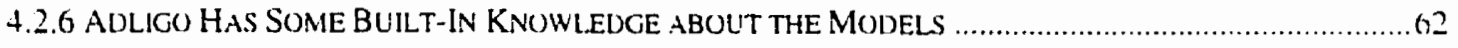

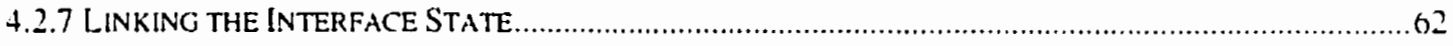

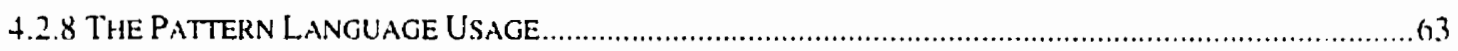

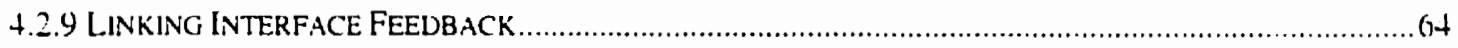

4.3 LINK GENERATION PROCESS: MORE DETAILS ....................................................................64

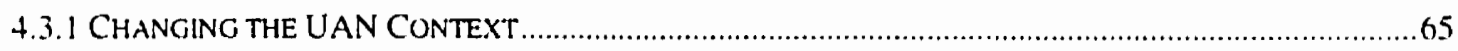

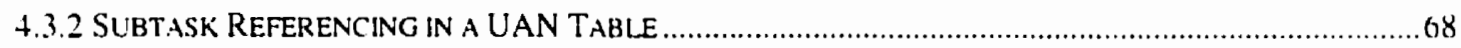

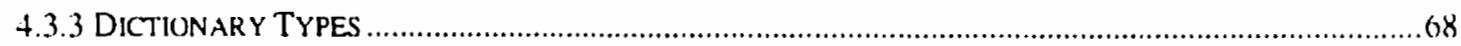

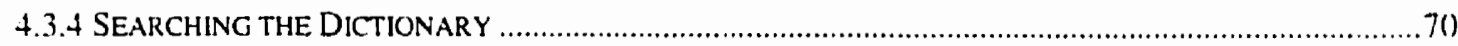

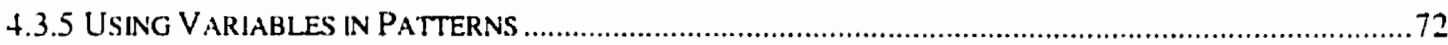

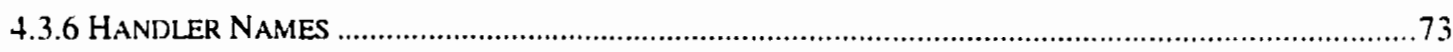

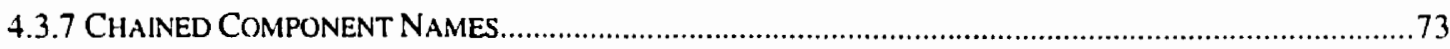

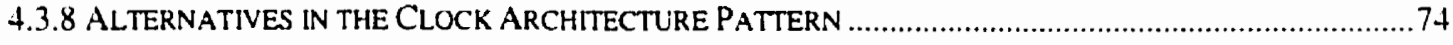

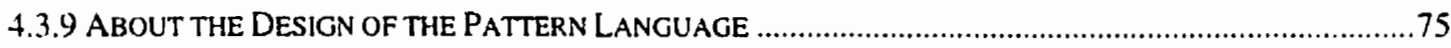

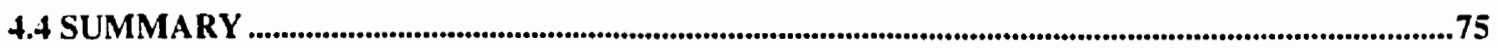


5.1 THE FRAMEWORK OF THE LINK GENERATION PROCESS …............................................79

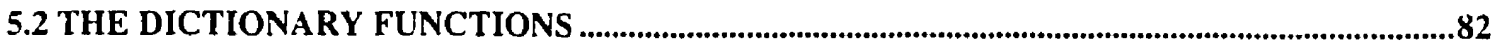

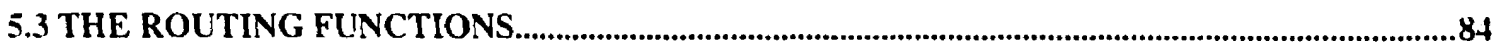

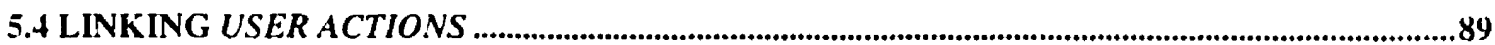

5.5 LINKING INTERFACE STATE AND CONNECTION TO COMPUTATION.................................91

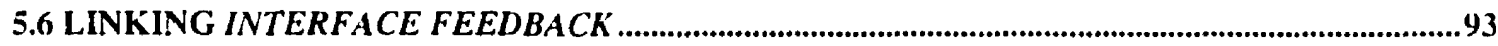

5.7 SUMMARY

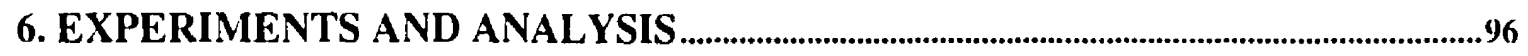

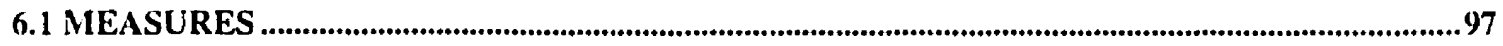

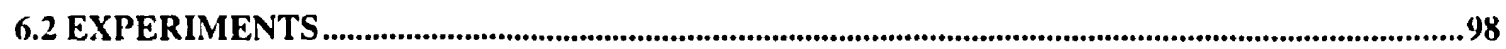

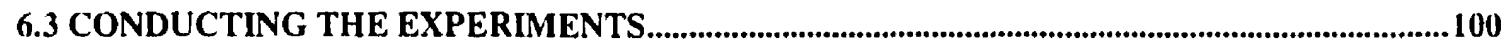

6.4 RESULTS

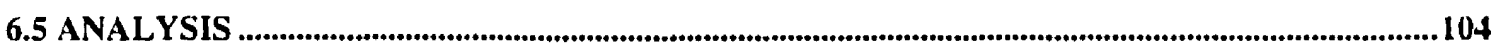

6.6 SUMMARY 


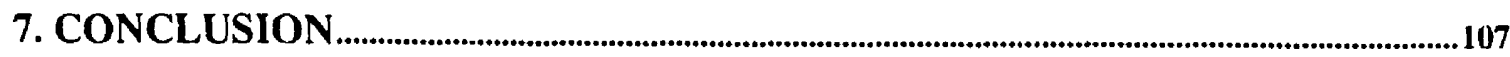

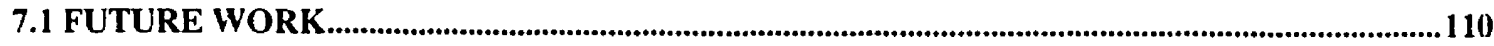

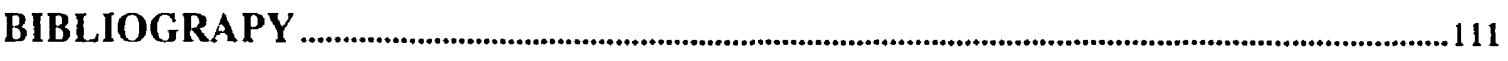

APPENDIX A. THE STANDARD DICTIONARY ...................................................................118

APPENDIX B. THE CPM PLANNER DICTIONARY ..........................................................119

APPENDIX C. THE VIDEO ANNOTATOR DICTIONARY ..........................................120

APPENDIX D. THE PATTERN LANGUAGE GRAMMAR...............................................121

APPENDIX E. THE UAN NOTATION GRAMMAR...........................................................124

APPENDIX F. THE CLOCK ARCHITECTURE FILE GRAMMAR .............................126

VITA 


\section{LIST OF TABLES}

Table 6.1 The Size of the Two Case Studies Used in Experiments...................... 98

Table 6.2 Results Collected from the CPMI Planner Experiment ....................... 101

Table 6.3 Results Collected from the Video Annotator Experiment .................1011 


\section{LIST OF FIGURES}

Figure 1.1 Coevolutionary Design of Interactive Systems.................................................2

Figure 1.2 Different Views Linked in Vista ...................................................................

Figure 1.3 Types of Links that Correlate Different Design Artifacts................................... 5

Figure 2.1 LiN Descripron for the Task "select a mode" .............................................11

Figure 2.2 Two Possible Presentarions of Task Types in ConcurTask Trees ..................... 14

Figure 2.3 The Lndo ()peration as a Task Spectied in ConcurTaskTree .......................15

Figure 2.t The Model-Viciv-Controller (MVC) Architecture ............................................ 21

Figrure 2.5 The Abstraction-L.ink-View . Nedel (basic strucrure) ........................................22

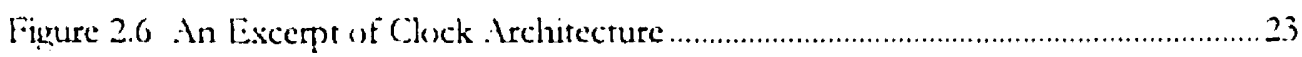

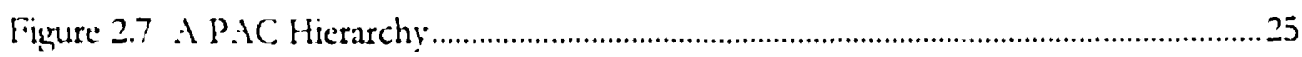

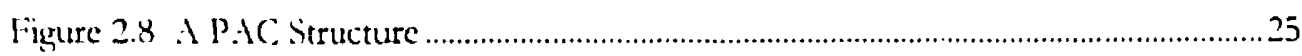

Iügure 2.9 Petri Net Representation for a Sequence of LAN Actions .............................28

Figure 2.10 Petri Net Representation for Choosing Berween LAN Actions..................... 28

Figure 3.1 Task Model for Planning a Project Using the Critual Path Method .................38

Figure 3.2 A Collaboration Tool: the CPN Planner ..........................................................

Figure 3.3 Interface Feedback of the Task "move a node" ................................................

Figure 3.4 Interface Feedback of the Task "select a mode" .........................................

Figure 3.5 Interface State of the Task "select a mode" .....................................................

Figure 3.6 UAN Description for the Task "move an object icon"...................................

Figure 3.7 The CPM Planner Clock Architecture ……..................................................

Figure 3.8 Linked Views in Vista (with manual annotations) .............................................48 
Figure 3.9 U.AN Contexts $\mathrm{n} 1, \mathrm{n} 2$ and $\mathrm{n} 3$ are implemented by "nodeView" 5)

Figure t.1 Framework of Generating Links Semi-Automatically .....................................55

Figure +.2 A Simple Clock Application, the Counter ………...........................................56

Figure t.3 The L.LN Description of the Counter Application .........................................57

figure 4.t Clock Architecture of the Counter Application ................................................. 57

Figure 4.5 The Generated Links of the Counter Application............................................

Figure t.6 The Dictionary Lised for the Counter Application............................................ j)

Figure 4.7 LAN Context " $n$ " translated to "node View" Component ...............................67

Figure 4.8 Jinking the "Reposition a Nude" Task to the CPM Architecture ..................69

Figure 4.9 Linking "currentilode $:="$ to . Mode.sethode ..................................................?

Figure 5.1 High-Level Design of the Link Generation Process...................................... 78

Figute 5.2 Generating a Link Between a LiN Match and a Handler .............................. 1

Figure 5.3 Illustrating LIN Context, Handler, ADT, and Component ..........................82

Figure 5.t Cieneraring Links for Liser Actions Column ..................................................86

Figure 5.5 Generating Links for Intertace State Column ................................................

Figure 5.6 Generating Links for Interface Feedback Column ............................................ 92

Figure 6.1 The Video Annotator Snapshot ..................................................................

Figure 6.2 Coverage Rate Analysis for the Adligo Tool.....................................................103

Figure 6.3 Error Rate Analysis for the Adligo Tool.......................................................103 


$$
\text { Cbapter } 1
$$

\section{INTRODUCTION}

Designing an interactive system consists of a set of processes such as task modeling, user interface design, task-oriented specification, and implementaton. Each process results in an artifact that expresses information contriburing to the usability of the developed ststem. Developers increasingly accept that such artifacts cannot be effectively creared separately, but instead coevolve incrementally, so that information obtained from the development of one artifact contributes to the development of the other [BRONN et al, 1998]. The need for a concurrent development is more apparent in designing user interfaces which demands iterattive refinement.

Figure 1.1 shows some of the design artifacts produced in the process of developing an interactive system. In this process, software engineers and Human Computer Intcraction ( $\mathrm{HCD}$ ) designers concurrently produce a set of design artifacts such as task hicrarchy (or model), user interface design (e.g., screens, mockups, and scenarios), task-oriented specification (in the U'ser Action Notation [H.tRTS(ON et al. 1990)), software architecture (e...., the Clock architecture style) and code (e.g., the Java language). 


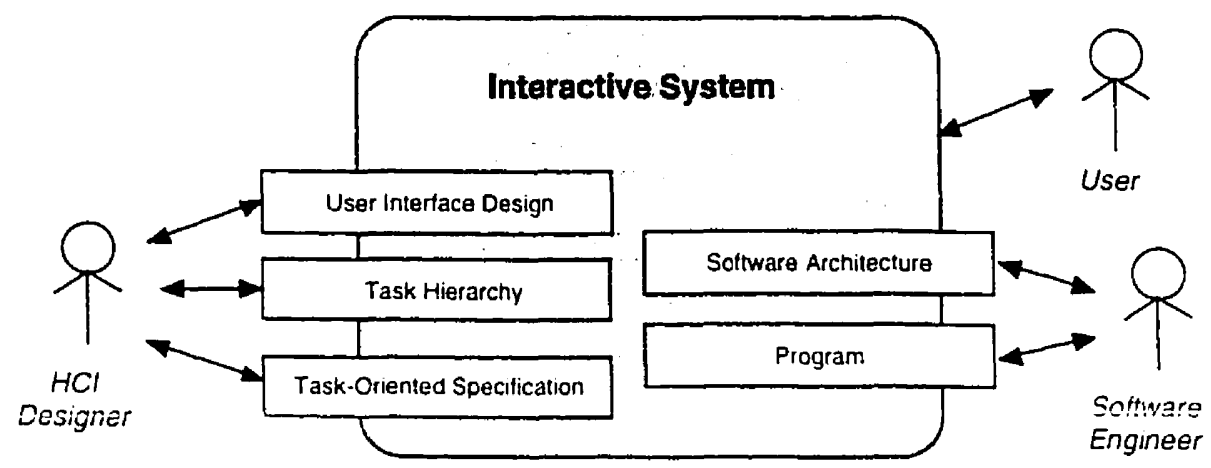

Figure 1.1. Coevolutionary design of interactive systems. The design process leads to a set of artifacts, which are developed iteratively and incrementally. Improvements in one artifact contribute to improvement in the others. Different artifacts represent the point of view of different participants in the design process [BROWN et al.. 1998].

Failing to relate the design ardifacts causes lack of communication between their developers. Correlaring information provided in the design artifacts allows the designers to see how knowledge from one design artifact can be applied to another. It is difficult to correlate design artifacts because they express information from different point of view (e.g., the users' view vs. the implementer's view) and at different levels of abstraction (e.g., code is much more detailed than architecture) [GRAHAN et al., 1996b].

To facilitate the communication berween designers, Brown et al. [BROWN et al, 1998) developed the l'ista tool which visually showed different user interface design artfacts linked rogether (see figure 1.2). 


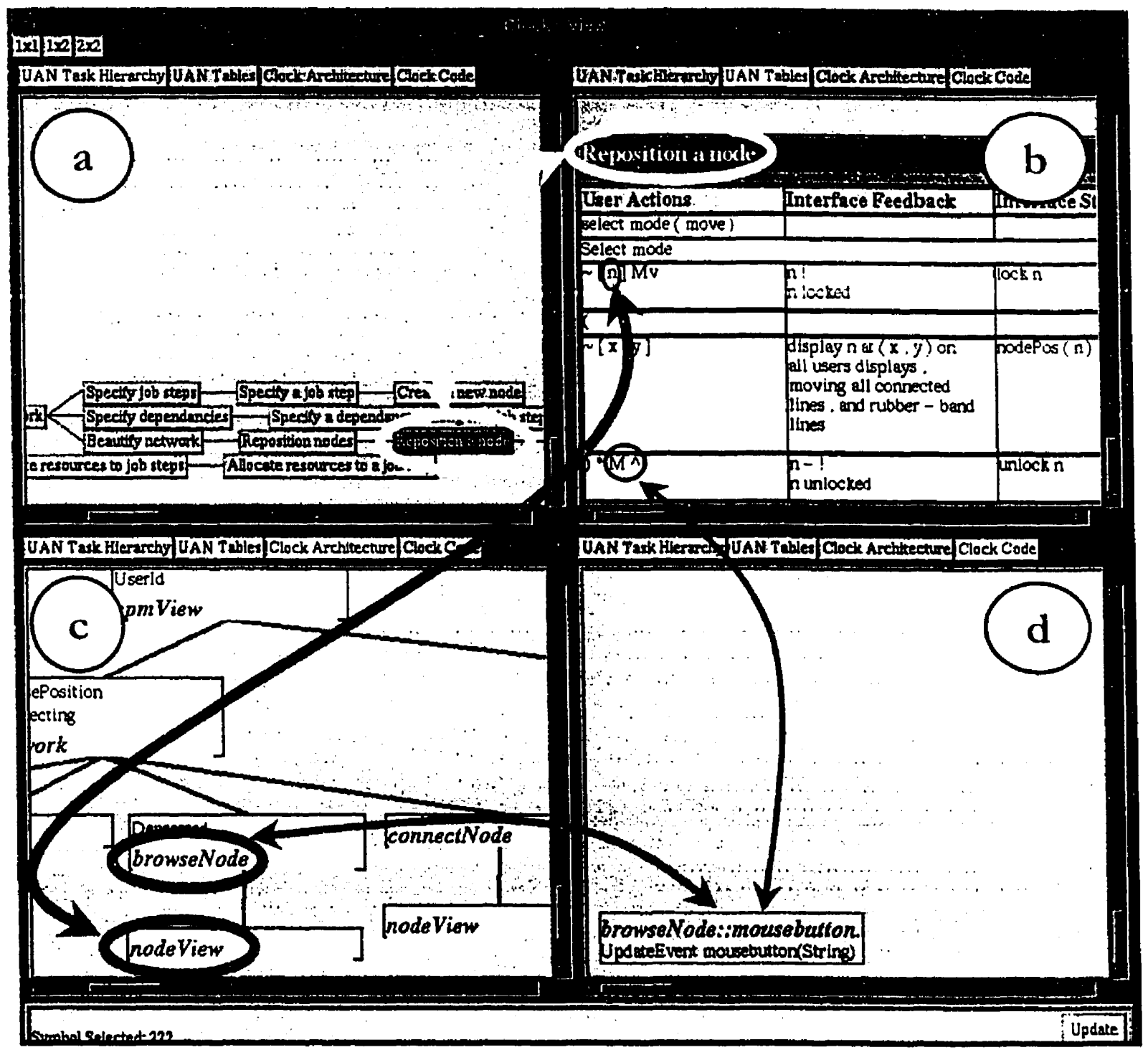

Figure 1.2. Different views linked in Vista: (a) the task model; (b) the task-oriented specification in the UAN; (c) the Clock architecture of the application, and (d) the Clock code. The Vista display has been manually annotated to show some of the links between the design representations. 


\subsection{Problem and Motivation}

Vista allows designers to simultaneously navigate a hyperlinked task hierarchy (model), taskoriented specification, software architecture, and code documents. However, Vista has one significant shortcoming. Links in Vista are hard-coded and specified by hand. In our experience, we may need to manually specify over 100 links for a medium stze case study (see chapter 6: Experiments and Analysis). The problem gets more challenging if we wish to maintain these links and validate them as the design artifacts evolve. In this case, the link generation process is necessarily iterative in order to maintain the correlation between the design artifacts.

To solve these two problems, we have to think of a new approach that facilitates creating and maintaining the links in a more automatic manner than the manual approach adopted in Vista.

\subsection{The Purpose of This Thesis}

Figure 1.3 shows the types of links that can be browsed using Vista. The current Vistal tool can automatically generate these links between the architecture model and the code (link 2), and berween the task model and the task-oriented specification (link 4). Generating these links is possible because the architecture model is a high-level design artifact for the code, and both lie in the construtional domain. Similarly, the task model is a high-level design artifact of the taskoriented specification, and both lie in the bebazioral domain.

Links $1,3,5$, and 6 are challenging to generate because they map artifacts lying in different domains. If we generate one type of these links we will be capable of mapping any design 

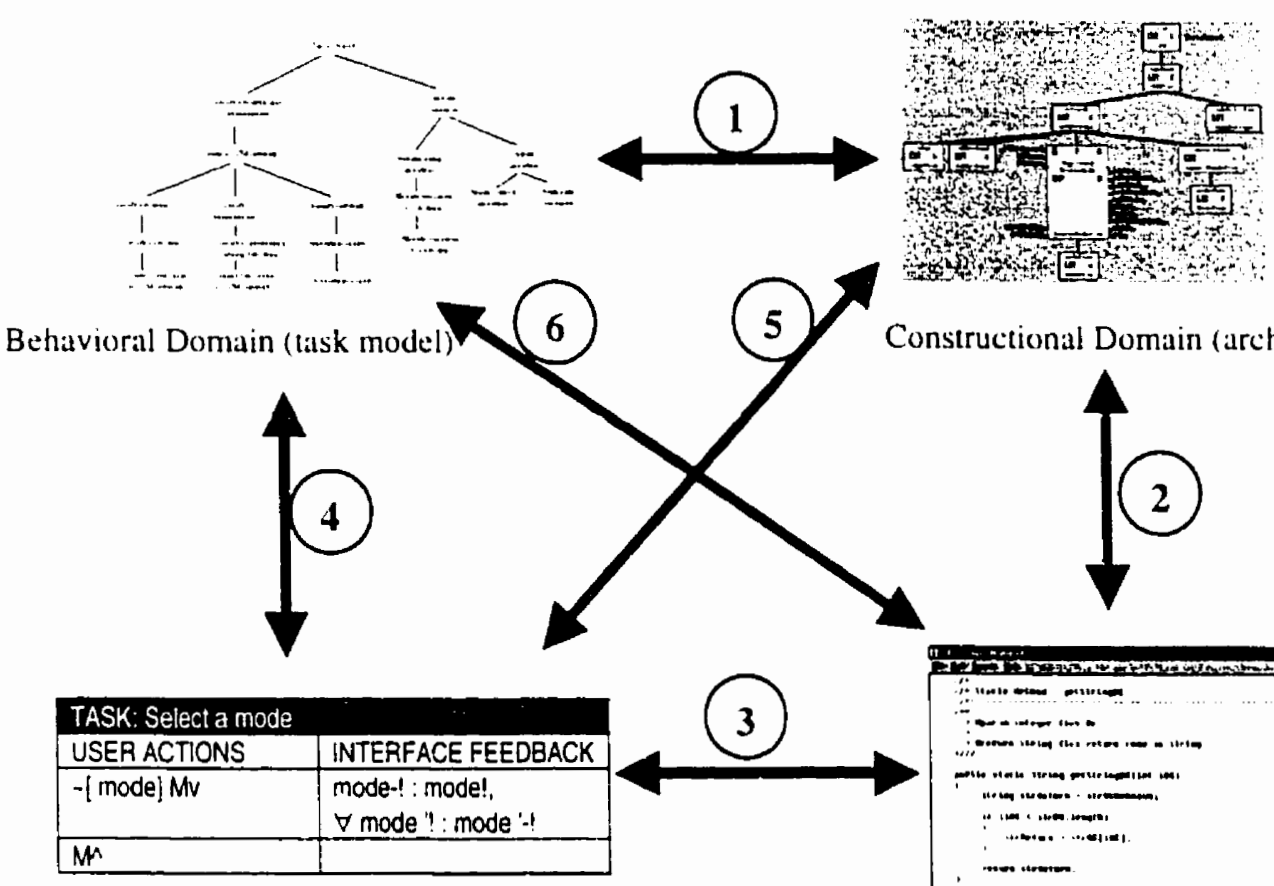

Behavioral Domain (task-oriented specifications)

Constructional Domain (code)

Figure 1.3. Types of links that correlate different user interface design artifacts. Generating links 2 and 4 is easy because they map artifacts lying in the same domain. Links I, 3, 5 and 6 map behavioral design artifacts to constructional.

ardifact to another through the other links. Accordingly, we chose to investigate how to generate the links between the architecture and task models.

We argue in this thesis that we can generate links between task and architecture models semiautomatically. We use the User Acrion Notation (UAN) [HARTSON et al. 1990] as a task model and Clock architecture [GRAHAil .AND LRNES, 1996] as an archirectural model. We could use other models in our work since they have many common features, as shown in the literature review presented in chapter 2. 
In fact, the U.NN specifications are far from implementation because moving from the L'AN to Clock archirecture requires a change in point of view, from the behavioral view of the $L A N$ to the architecture implementation. As such, the main issue of mapping one of these models to the other is the change in point of view, not the change in level of notation.

In addition, the Clock architecture (which is used by software engineers) can be easily processed by a compurer-based tool due to its well defined formality [GRIHANM, 1995], while the LAN descriptions (used by HCI designers) contain prose [HARTSON et al. 1990) which is hard to process automatically.

\subsection{Our Approach to Solving This Problem: Adligo Methodology}

In this research, we demonstrate how different design artifacts can be linked semiautomatically by developing . Illigo'. a prototype tool for generating links between task and architecture models with human assistance. This assistance is represented by a newly introduced artifact called the ditionary, which translates the U.AN names to the Clock architecture names. As opposed to the traditional linguistic dictionary, this dictionary contains mapping rules, which are written in a simple patterm language we have designed for this purpose (see chapter 4 ).

However, the solution for linking the UAN model to its corresponding Clock architecture is not a naive translation from one notation to another. Rather, it is a matter of cransforming the behavioral view, represented by the $U A N$ task model, to the constructional view represented

\footnotetext{
1. Illigo is a Laan word, which means, "I connect".
} 
by the Clock architecture model. Therefore, in addition to the dictionary, Adligo needs a few internal hardwired rules (see chapter 5) to be able to generate sufficiently correct links.

Through these rules, Adligo becomes semi-automatically able to derive links berween the models. We used the l'ista tool [BRowN et al., 1998] to graphically browse the generated links between the LAN notations and the Clock architecture (see chapter 3).

\subsection{Results and Analysis}

In order to get a sense of how well Adligo performs, we focused in our experiments on two Cilse studies: the CP.M Planner and the V'ideo -Amotutor applications (see chapter 6). ()ur results show that in these two case studies, we were able to generate $90^{\%}$ o of possible links with error rate of 1$)^{\%} \%$ to $12 \%$ with only 8 rules in the dictionary. However, we believe that further experiments are required to determine whether these results generalize to applicanons of Adligo.

\subsection{Contributions}

The contributions of our work are summarized in the following points:

1. We developed a method to semi-automatically derive links between task and archirecrure models. This method has been demonstrated by a computer-based tool called Adligo. 
2. We developed a new computer-based tool (Adligo) for generating links between the LAN task model and the Clock architecture model. This tool is fully integrated with the 1 ista tool which visually exposes the hyperlinks generated by Adligo.

\subsection{Road Map}

Chapter 1: Introduction gives a research overview, problem statement and motivatuon, the provided solution (Adligo's methodology), list of contributions, a summary of the results, and a road map for this thesis.

Chapter 2: Reluted It"ork provides a literature review. The chapter consists of three main parts. The first describes the task models and gives several examples for task modeling rechniyues. The second is concerned with describing architecture models commonly used in software engineering. This survey of models alerts us to their common characteristics. The third part reviews some efforts similar to our work.

Chapter 3: Design . Irtifacts Browsed in l'ista explains the UAN notation, how it is used ats a task model, and how it is employed to write task-oriented specifications. This chapter gives also a detailed description of the Clock architecture. Finally, it describes the liata tool, which is the motivation of our work.

Chapter 4: Semi-Automated Link Gentration Process: The Adligo Tool explains in detail the link generation process for Adligo users. It describes the general framework of the process and gives an introduction tutorial for generating the links by Adligo. This chapter discusses many 
details related to new concepts presented in this research such as the dictionary and the patterm language.

Chapter 5: Looking Linder the Hood: The Implementation of - Ldligo explains: technical aspects related to Adligo's implementation. The discussion in this chapter is supported by formal specification for the functions used in Adligo's methodology:

Chapter 6: Experiments and . Analysis provides an evaluation of the Adligo tool. It gives at description of the experiments we performed. In this chapter, we determine the measures by which we evaluate Adligo's performance. Also, we discuss our results and analyze them.

Chapter 7: Conctesion highlights the main ideas discussed in this thesis and gives directions for future research. 


$$
\text { Cbapter } 2
$$

\section{RELATED WORK}

In this thesis, we show that generating links semi-automatically between task and architecrure models is possible. This chapter provides a literature review of some task and architecture models, and a review of similar efforts to link them together.

The chapter consists of three parts. The first describes four task models commonly used in designing user interfaces. The second part describes four architecture styles used in the software engineer community. The last part describes some efforts similar to our work.

The work in this thesis is based on the hierarchical form of task model. We survey this class of task model, but base our work on a single representative, the Liser Acrion Notation (LAN). Similarly, a wide range of architectural style for user interface development are based on layered variants of the model-view-controller style. We survey these architectural styles and choose the Clock architecture style as a representative on which to base our work. We believe that because these models (UAN and Clock) are representative of wider classes, the techniques presented in this thesis may have wide applicability. 


\begin{tabular}{|l|l|l|}
\hline \multicolumn{2}{l|}{ TASK: Select a mode } \\
\hline USER ACTIONS & INTERFACE FEEDBACK & INTERFACE STATE \\
\hline$\sim$ mode] Mv & $\begin{array}{l}\text { mode-! : mode!, } \\
\forall \text { mode'! : mode-! }\end{array}$ & selected = mode \\
\hline$M^{\wedge}$ & & \\
\hline
\end{tabular}

Figure 2.1. UAN description for the task "select a mode". The table show's the interface feedback and its state as a response to the user actions.

\subsection{Task Models}

A task model records the tasks that potential end-users of the system may need to pertiorm to do their jobs, independent of dealing with a particular computer system. The design of an interactive system is based on supporting these tasks. This secrion describes some taskmodeling techniques. We conclude this part by a summary in which we report the common fearures among these models.

\subsubsection{The User Action Notation (UAN)}

The L'ser Action Notation (LAN) [HARTSON et al. 1990)] is a task-oriented notation rhat describes the behavior of the end-user and the interface as they perform a task together. The LAN was initially intended to be the communication means between interface designers and the implementers. We use the UAN to describe task models (see section 3.1) and task-oriented specificarion encoding how tasks are carried out with the user interface, from the point of view of the end-user. HCI designers use the UAN to encode their tasks in a tabular format (see figure 2.1). 
L'AN symbols are meaningful mnemonics and can be typed using a standard keyboard. The LAN notation supports temporal relations between tasks [H.ARTSON AND GRAY, 199()].

The LAN provides rask coverage as it shows whether all the intended system rasks are supported in a reasonable and consistent way. The LAN explicity shows which tasks are supported by the system and consequently helps in understanding how the system fits within the context of its use.

In comparison to specifications using scenarios, mockups or prose, the L'AN provides higher precision and accuracy. This is especially urue of specifications of the concurrent properties of user interfaces which are important to groupware applications.

L'AN has, however, the following disadvantages [GR.IH.LM er al., 1996b):

Ciumbersome and Tedious: UAN specifications for complex systems can run over dozens of pages. This makes reading difficult and scarching for subtasks a hard job.

Diffizult to Maintuin: Changes in a user interface design can require considerable change in its corresponding L'AN specification. Provided that design is always cvolving, the LAN is rypically left behind as designers become unwilling to spend the time updating it-

These two points are addressed through a browser [Telford, 1996] which supports easy navigation of the UAN specifications. Such a browser may evolve to a complete editor.

Hard to Transform to Implementation Domain: The linkage between the LAN specifications and their implementation is not clear. Some studies have been done to bridge the gap between 
task-oriented specification and implementation [GR+H.MN et al., 1996b]. Our work in this thesis falls in this category as we cry to link the UAN task model with the Clock architecture model.

\subsubsection{ConcurTaskTrees}

Concur TaskTrees is a graphical notation for describing tasks with a wide range of temporal relationships. The task in this approach is descrbed by the following attributes [P.ATER.N() et al., 1997b|: Name, Type, ()bjects, First Action and Last Acaon. To construct the task specification, objects must be identified and communiation actions must be defined. There are two types of objects:

Pencimable Objects: items which end-users can sense, such as menus, icons, windows, voice, sounds, and so on. They can belong to application or inceraction tasks.

Intermal Objetts: entities which belong to the application, like the database.

Actions can be cognitive, logical, or physical. We can identify four types of tasks:

Csur tasks require cognitive or physical activities without interaction with the system. For instance, when end-users watch a video clip and decides which frames they are interested in for annotation, they are performing a user task.

Application taskes are completely activated and execured by the system. For example, detecting and displaying errors during compilation or during monitoring the network. 


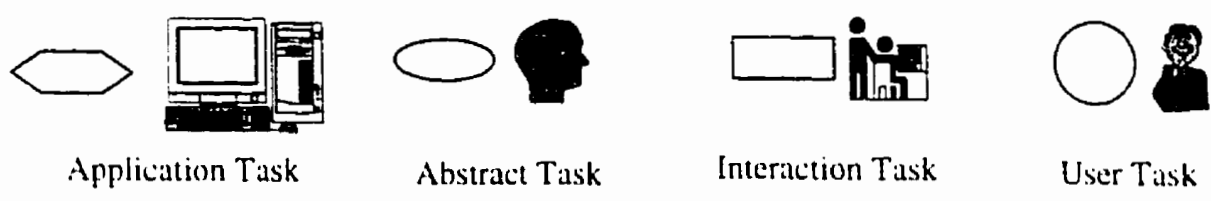

Figure 2.2. The two possible presentations (shape or icon) of task types.

Interation test are invoked by the end user and imply his or her interation with the system. For example, editing a document or phrasing a query to a database are interaction tasks.

- 1bstrut tustes require complex actions and are classified as none of the above.

In a ConcurTaskTree task specification the types of tasks are presented differently either by icons or geometric tigures (figure 2.2):

The task model is built in three phases. A tree-like structure is built using a herarchical logical decomposition of the tasks. In each level of that tree, we identify the temporal relationships among tasks; for each layer, we identify the objecis and the actons which allow them to communicatc.

ConcurTaskTrees allow designers to describe concurrent tasks [P.TTERV() et al., 1997b], unlike GOMS [CARD et al, 1983], which uses hierarchical task decomposition to analyze sequendal tasks only. Figure 2.3 shows a simple example of a task specified by ConcurTaskTree. We have a generic application during which the end-user can edit vatious possible modifications. ()nce a modification has been specified the end-user can either perform it or cancel it. In borh cases, as editing is an iterative task, the end-user will be able to specify a new modification until the close task is performed. 


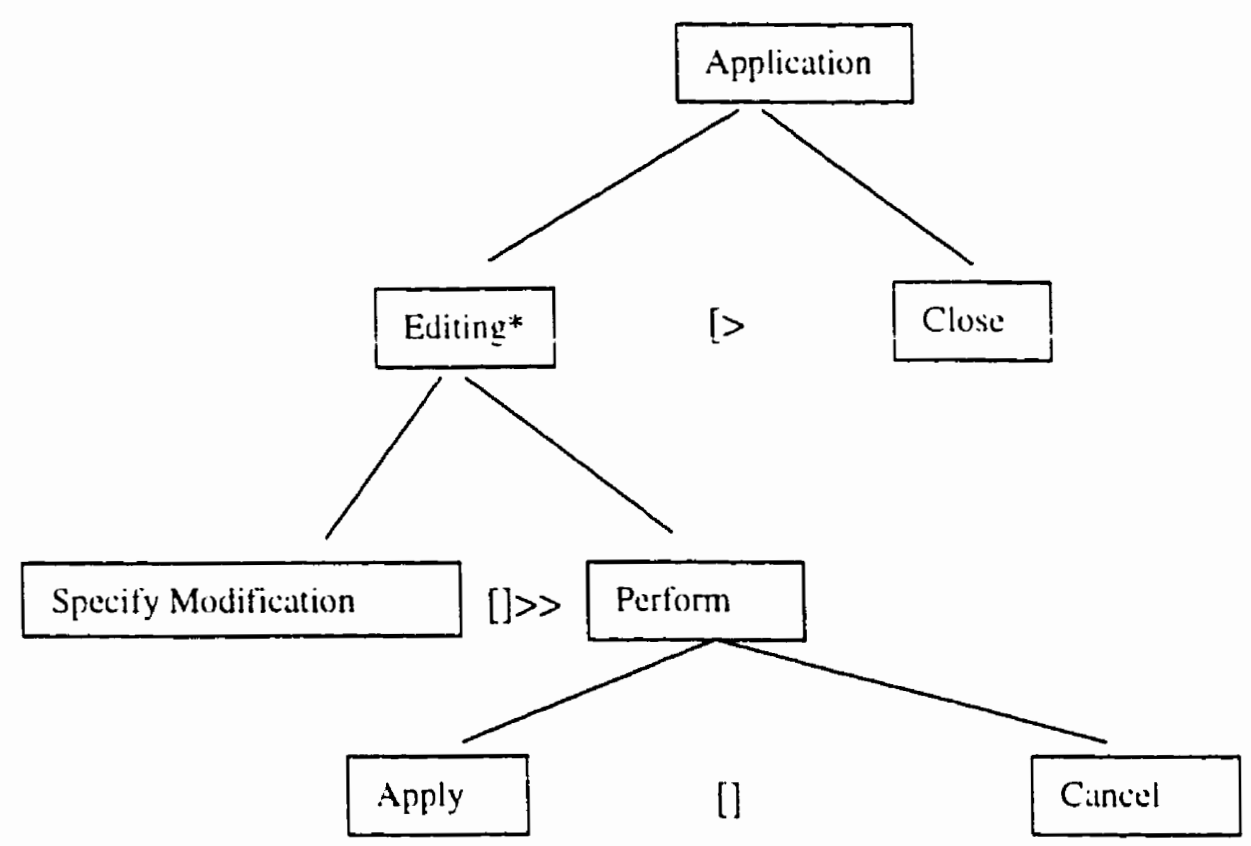

Figure 2.3. The Undo operation as a task specified in ConcurTaskTree.

We may nodec that ConcurTaskTrees are more formal than the L'AN because they are more systematic, less ambiguous, and require more details to be specified in order to provide adequate task coverage.

\subsubsection{The GOMS Model}

In the GOMS model [CARD et al, 1983], the end-user's cognitive structure is composed of four elements: (1) a set of Goals, (2) a set of Operutors, (3) a set of Methods in order to achieve the goals and (t) a set of Selection rules. 
Goals: A goal describes the aim which the end-user wishes to do and it determines a set of methods needed to achieve that goal.

Operators: These are steps whose execution is necessary to change any aspect of the enduser's mental state or affect the task environment. However, a G( Mis' model does nor support any tine structure of concurrency [CARD et al, 1983]. Behavior is described through the serial execurion of operators. In operator is defined by its effect (output) and its duration.

Method: This is the procedure needed to achieve the goal. A method can be specified as a conditional sequence of goals and operators.

Control Structure (Selection Rules): In order to reach the desired goal, there might be more that one method aviulable to the end-user. In the GOMSS model, method selection is handled by at set of selection rules. Each selection rule is of the form "if condition is true in the current rask situation, then use method M."

For example, in the process of building a text editor application:

GONL: Del-:All-Text

CTRL-A-COMMLAND

DEL-COMMLAND

Here, the goal is Del-All-Text, and the block defines the method to achicve it. Both CTRL-ACOMMAND and DEL-COMMAND are operutors. U'sing this block structure, designers can create a hierarchy of end-user tasks. 
The amount of detail generated in a GOMS description of an interface allows for dhorough analysis. On the other hand, it can be a significant undertaking to produce.

\subsubsection{The Task Knowledge Structure (TKS)}

1 Task Knowledge Structure (TK.S) [Johnson et al, 1988] summarizes a end-user's task knowledge. This information guides the designers in making decisions about what knowledge posisessed by the end-users will affect their interaction with the system and how a design solution might require the end-user to acquire more knowledge, or, conversely, of no longer require certain knowledge. In addition, such information about end-users' task knowledge will help to identify what should be supported, and what will cause problems and crrors en occur, and so on. A TKS description is constructed by a member of the design team, whe can be either an HCI specialist or a system designer.

The TKS' requires the following knowledge components for a given task: roles, goals, subgorals, subtasks, plans, procedures, strategies, acrons and objects. A role is assumed to be defined by a particular see of tasks that an individual is responsible for performing as part of their dury in a particular social context. A goal is assumed to be the state of affairs that a parncular task can produce and forms part of a person's conceptualization of their task world while carrying out at given role. A plan is the particular formulation and possible ordering of subtasks that are undertaken to achieve a particular goal. A procedure is a particular element of behavior that forms part of a subtask.

Actions and objects are the lowest level of elements of tasks and are the constituents of procedures. The highest level of representation of the model is provided by the role/task 
relations. The next level is the content of the TKS for a given task. This representation includes relations to lower level representations of the model containing the plan, procedures and taxonomic substructure (i.e., action and object property/feature lists). At a lower level of representation in the model is the plan and procedural knowledge associated with the specific task. 'This representanon is referred to as a goal-oriented substructures. The plan represents the structure of the subgoals and the states which those subgoals sadisfy. Where there is more than one alternative set of procedures these constitute different strategics. The procedures also have links to the taxonomic substructure, which is the lowest level of representation in the model.

\subsubsection{Summary}

From our survey, we can see that task models may vary in the following points:

- Formulism: The formality degree of one model varies trom the other. For example, LAN descriptions may contain prose, while ConcurTask Trees are very formal. The formality if the model governs the flexibility of processing it automatically. 1.e., processing Concur Task Trees is easier than processing LAN.

- Temporal Relationship: Some task models (e.g., UAN and ConcurTaskTrees) support temporal relationships while others do not (e.g., GOMS).

- Task Representation: The task representation might vary from one model to another. For example, in the UAN, a task is represented by a UAN table, in the ConcurTaskTree as an object and in GOMS as a goal with its own block.

While we notice the following common features among them: 
- Ljer Gout. A task model starts by a goal to be achieved. The model describes how to achieve this goal from the end-users' perspective.

- Task Coteruge task models show whether all the intended system tasks are supported in a reasonable and consistent ways.

- Hierarthical Tree Task models adope the hierarchical tree representadon ro describe the decomposition of end-users' goal and the tasks needed to achieve this goval. 'This hierarchical tree may be in different presentadions. For example, a Concur latsklirec explicitly presents its model graphically, while GOMS uses indented blocks, or implicitly uses subtask references as with the L'AN.

- Tasks: The basic element in any task model is the task.

- HCI designers: task models suit the HCI designers who might not be computer specialists, but instead artists, psychologists or experts in designing usable interfaces.

- The Need for Compater-Based Tools: most of the task models (c.g., LAN, Concurlatiklrees, GOMS, ThS) can be tedious to produce if they are not supported by a computer-aided tool. 


\subsection{Architecture Models}

Software engineers decompose complex systems into small parts using software architectures. This reduces the complexity of implementation, cvolution and maintenance of the sistem [GRAHAM AND URNES, 1996].

The majority of architecture styles are based on the same separation of user interfaces and application seen in the Seeheim model [PF.AFF et al., 1985]. However, where Seeheim proposes dividing the entire system into a single applicarion and a single user interface, these styles divide the system into fine-grained subsystems, each with its own applicaton and user interface component. Such architecture styles include: Model-View-Controller (MVC) [KRASNER AND POPE, 1988], Clock [GRAHAM .LND L'RNES, 1996], Abstraction-Link-liew

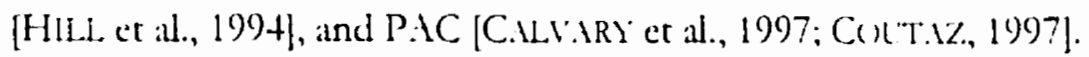

Most of the architectural styles discussed here are supported by programming languages or toolkits and all have been used to develop interactive applications.

\subsubsection{Model-View-Controller (MVC)}

This architectural style was introduced in Smalltalk76 [KRASNER . WND POPE, 1988] and has been implemented with minor variations in a wide range of user interfaces toolkits, most recently in Sun's Swing framework for Java [ECKSTEIN et al., 1998]. 


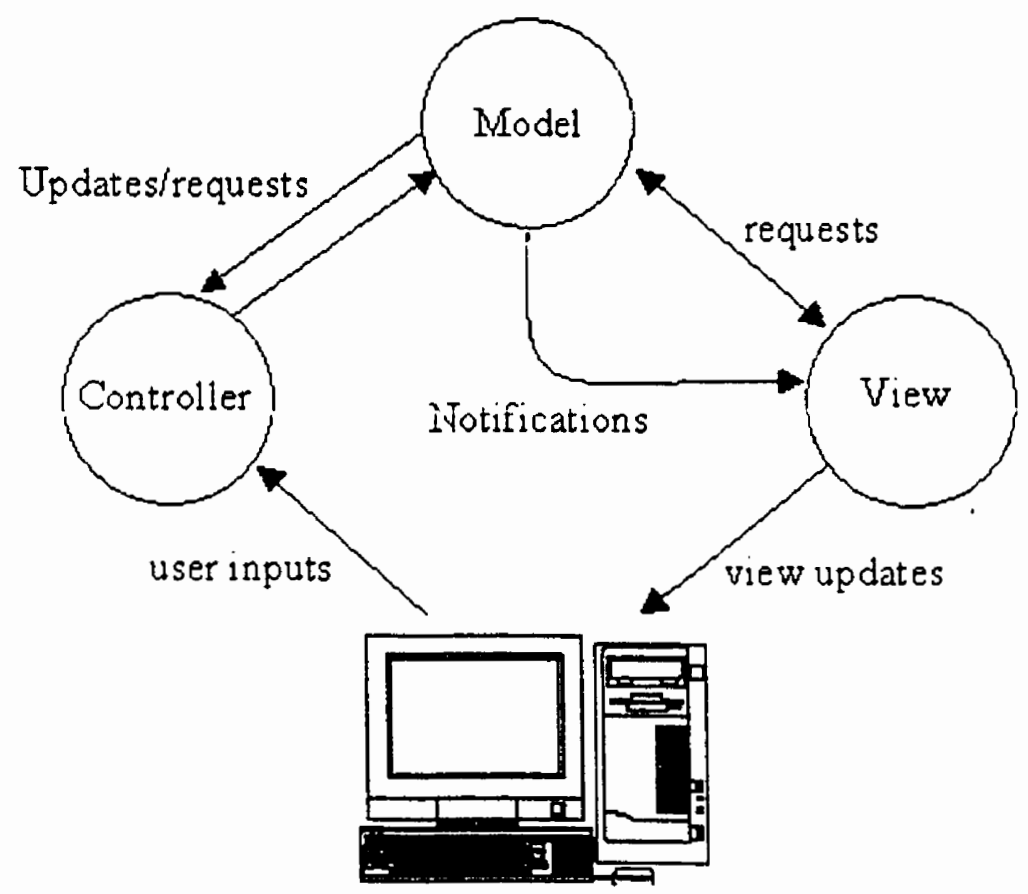

Figure 2.4. The Model-View-Controller(MVC) architecture [KRASNER AND POPE, 1988 ].

Figure 2.t illustrates the structure of MVC. An MIVC triad consists of a model, which represents the application's data; a controller, which interprets end-user input; and a wien, which presents output.

The view and the controller can send updates and requests to the model. In response to state changes, the view presents a new output to reflect the new starus of the model.

The key to the MVC's power is how these components communicate. The controller receives and handles the end-user inputs. It then determines how these inputs should be reflected as modifications to the model. The model then announces to the view that the model has changed. The view then updates itself, resulting in a new display for the output. 


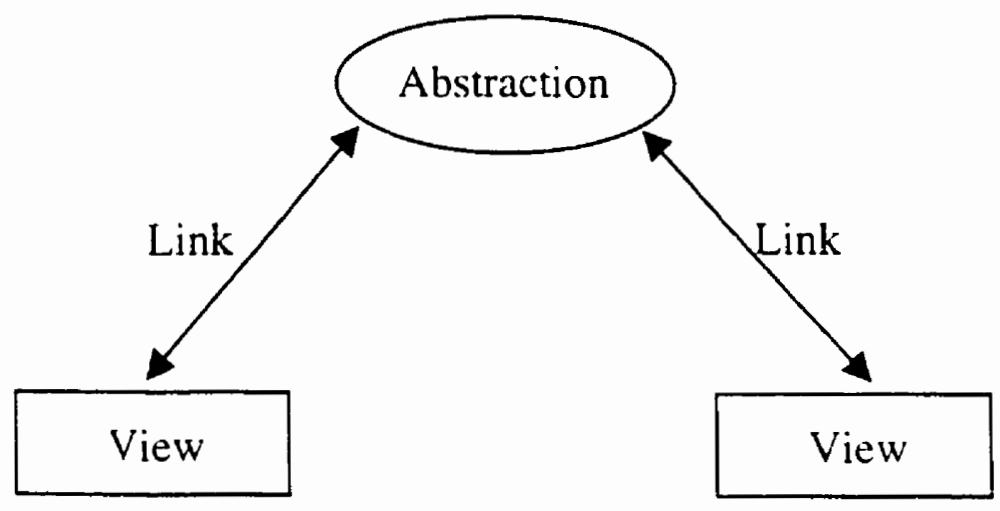

Figure 2.5. The Abstraction-Link-View model (basic structure).

This approach leads to a strong separation of concems between the model, view and controller. The controller does not directly communicate with the riew, only with the model. The view is responsible only for updang the display, and does not need to know the detals of when display updates arisc.

\subsubsection{Abstraction-Link-View (ALV)}

The Abstraction-Link-View architectural style ( $\mathrm{H} \mathrm{V}$, pronounced "al-vee") $[\mathrm{H} 1 \mathrm{~L}, 1992)$ was developed at Belleore, and is based on MVC. ALV consists of combined view-controller pairs (called miews), separate hierarchies of views and abstractions (models), and a constraint mechanism called the link which connects the two. Abstractions and views are trees of ubjects; links are "bundles" of constraints. The ALV architecture style is shown in figure 2.5.

The major difference between ALV and MVC is ALV's use of constraints instead of calls and events to connect the view and abstraction hierarchies. Neither abstraction nor view requires knowledge of the other because the constraints mediate all communication between them. 
$\mathrm{ALV}$ constraints are completely declarative, removing much of the inherent complexity associated with callbacks [MILERs, 1991].

\subsubsection{Clock}

The Clock architecture style was designed to support the evolutionary design of architectures for interactive, multi-user systems [GRAH.LM AND L'RNES, 1996]. The language provides the properties of restrited sioping to reduce direct dependencies among components, autumutic message routing ina dolegation, and easy modification of the bieranthial strutures of architectures. Clock is supported by the visual Clockllorks programming environment [GRtHtil et al., 1996a].

Figure 2.6 shows an excerpt of the Clock :urchitecture for a sclected progr:um. I Clock

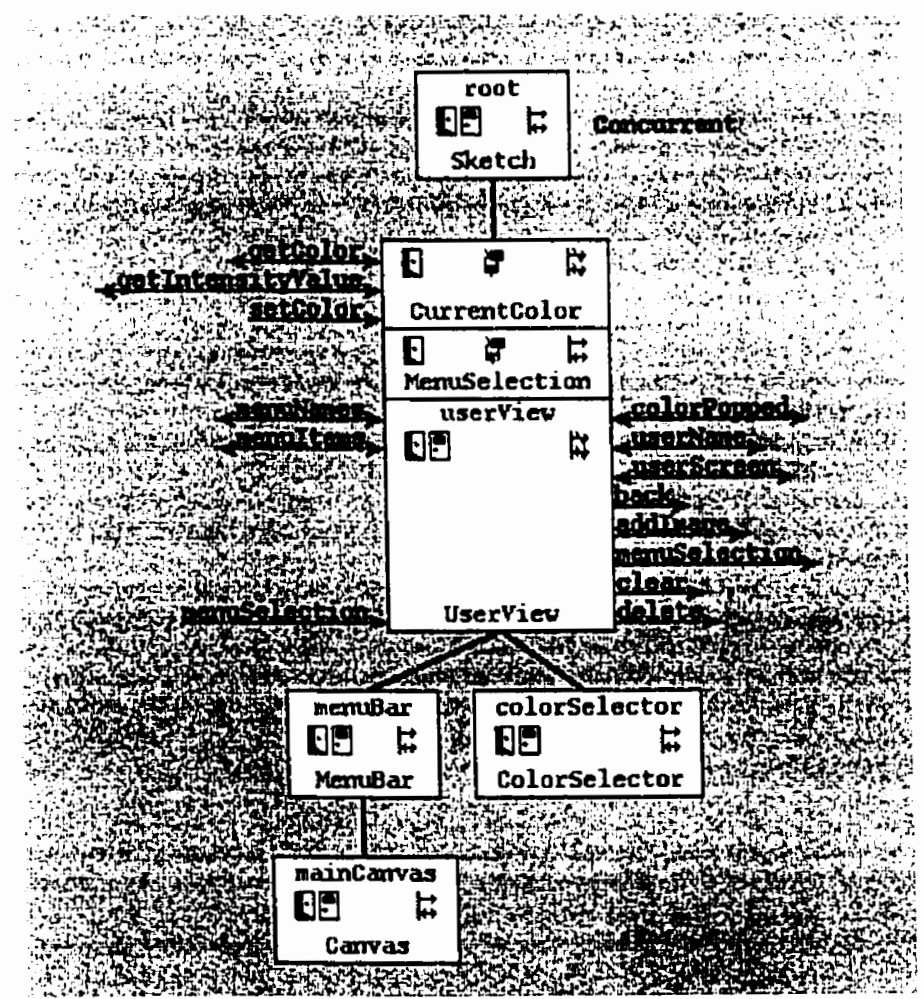

Figure 2.6. An excerpt of Clock architecture. 
architecture consists of a tree of communicating components. Components may respond to end-user input (or input from other components), and may produce graphical ourput to be placed on the display. Components may also contain instances of abstract data types (. 1DTi), such as the CurrentColor attached to the userl 'itw component. Each component has a name (e.g., colorsielector) and a class (e.g., ColorSelector).

Components communicate via interface messages: input, request and tpdete messages. Messiages are automatically routed up the tree to the nearest component capable of handling them. This routing mechanism provides a form of inheritance by delegation, where components inherit all the facilities of the components appearing above them in the tree. This communication mechanisms means that components can only access the data of components appearing above them - components may not directly communicate with their children or siblings.

\subsubsection{Presentation-Abstraction-Control (PAC)}

The Presentation-Abstraction-Control (P.AC) architecture style [C.LLV.IR] et al., 1997; ColTAZ, 1997], decomposes a system into a hierarchy of PAC triads. Each triad includes a presentation, which is analogous to an MVC view-controller pair, and an abstrution, which is analogous to an MVC model. However, where MVC views and controllers communicate directy with their models, all communication in a PAC triad is mediated by the control. This has the key advantage that presentations and abstractions can be developed in isolation from one another, since any syntactic or semantic mismatches can be accommodated by the control. $A$ PAC triad is shown in a figure 2.7. 


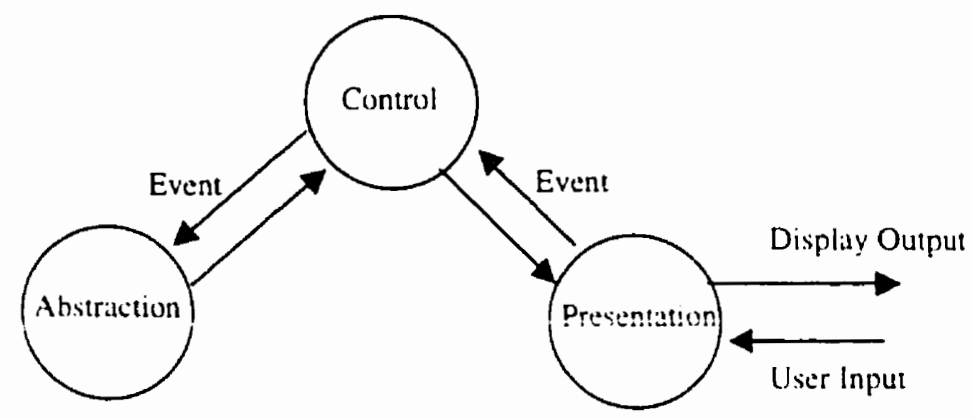

Figure 2.7. A PAC hierarchy.

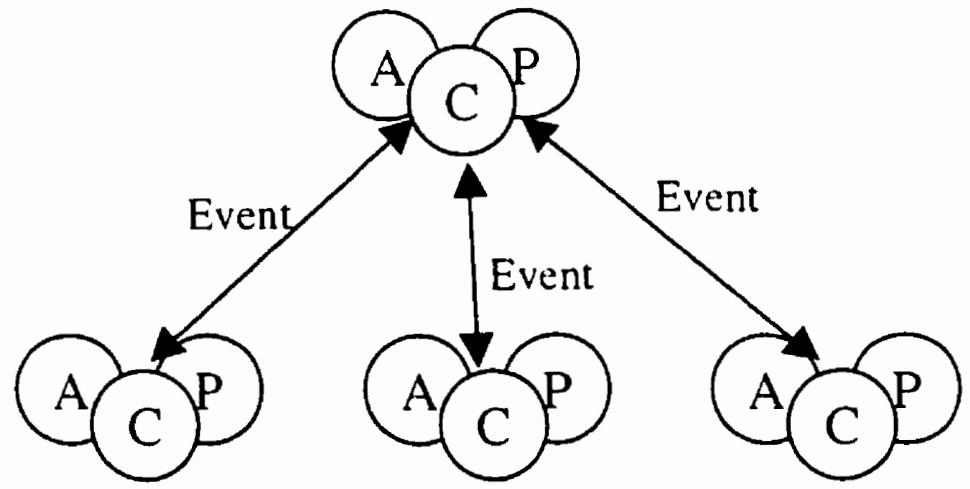

Figure 2.8. A PAC structure .

Figure 2.8 illustrates the structure of a typical P.AC application. The hierarchy of P.AC triads represents application composicion. The root triad represents the applicarion as a whole, and is decomposed (here) into two subcomponents, which might represent different windows of the application. These are in turn further decomposed until the level of individual interactive objects is reached. 
In the same way that PAC controls mediate communication benween their presentadions and abstractions, they also mediate communication up and down the hierarchy. This keeps the presentations and abstractions simple, and allows the controls to approprately update their presentations and abstractions in response to in formation arriving from higher to lower levels.

\subsubsection{Summary}

From this survey, we can summarize the common features among these architecture models in the following points:

- Most of the architecture styles have the powerful feature of the separation of input and output (inherited from the MVC model).

- Each architecture style is represented by a hierarchical tree of communicating components. However, component details may vary from one architecture style to another.

- Each architecture style has its own mechanism of automatic update of the display whenever the model changes (e.g., by constraints like Clock, links in $\mathrm{ULV}$, or atents in P.AC).

\subsection{Efforts to Bridge the Gap}

In this section, we briefly describe some rescarches similar to our work in terms of exploring the theme of linking the behavioral domain the constructional one. Section 2.3.1 describes an approach in which the UAN model was transformed to Petri nets, giving formal meaning to the UAN. Section 2.3.2 describes a methodology by which architecture models can be derived from ConcurTaskTrees. Section 2.3.3 describes a complete environment (ADEPT) which 
accepts a task model (TKS) as input and constructs a complete user interface out of it. Siccrion 2.3.4 describes a framework in which mulaple perspectives are maintained separarely as distributed objects, called l'itwPoints.

\subsubsection{Transforming the UAN to Petri Nets}

The gonl of this work was to provide a gateway between tast models defined with the L'IN formalism and system models.

That wals accomplished by giving formal perri net equvalencies for every LAN construct [P.LLAN(XLE et al., 1995]. Petri nets are a mathematically founded formalism designed tiur modeling concurrent systems. When modeling with Petri nets, a system is described in terms of state variables (called places, depicted as ellipses) and by state-changing operators (called transitions, depicted as rectangles), connected by ans. The state of the system is given by the marking of the net, which is a distribution of tokens in the net's places. State changes result from the tiring of transitions, yielding a new distributon of tokens. Transition firing involives two steps: (1) tokens are removed from every input place, and (2) new tokens are deposited in every output place. A transition is enabled to tire when all of its input places contain tokens. Figure 2.9 depicts a small Petri net which illustrates the simple concept of sequences of actions. 


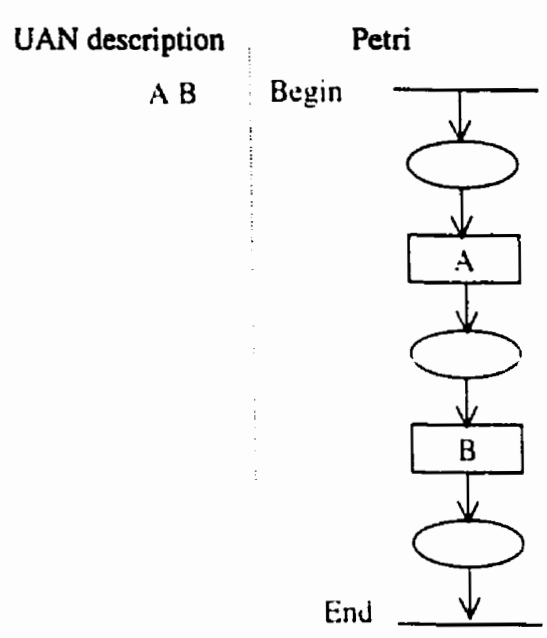

Figure 2.9. Petri net representation for a sequence of tasks $A$ and $B$ described by the UAN notation.

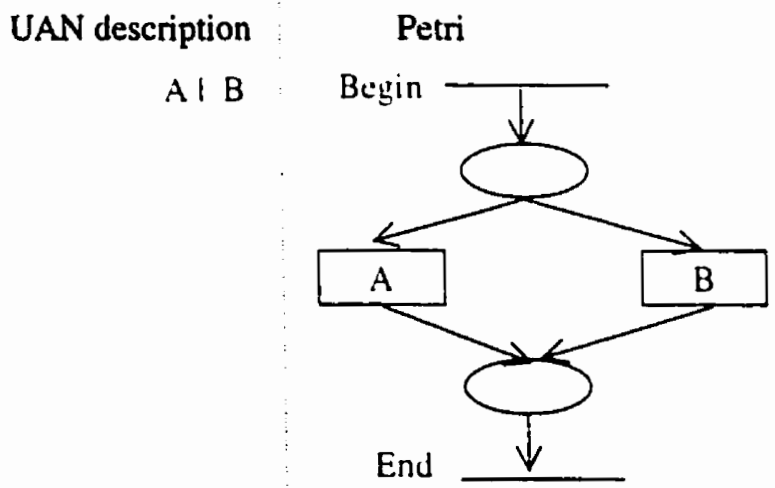

Figure 2.10. Petri net representation for the UAN description of choosing between two tasks $A$ or $B$.

Figure 2.10 indicates another relationship which is a choice between tasks when the enduser has to choose (equally) and perform one of these tasks. 
One of the benefits gained from this work is resolving the ambiguiries of task models during the translation process. This process, while automated to a certain extent, is interacrive as it asks the designer of the tasks models to make their specification more precise if needed.

\subsubsection{Engineering Task Models}

Paterno and his colleagues [PATERN() et al., 1997a] described a method which allows designers and developers to define a task model of the interactive application considered and then to derive an architecture specification. The goal of the work was to obtain a sistematic method that allows designers to derive architectural models from task models.

This work was based on the ConcurTaskTrees as a task model and on an architectural model which differs from other models tor sofnware interaction objects such as PAC or Myers' interactors [NIERs, 1990], as it structures cach of them as an entity supporting bi-directional communication between users and software applications.

The work focused on identifying rules for transforming tasks to interactors. ()nce these rules have been precisely defined, they can be incorporated into a tool which supports the transformation of task models to architectures. However, this group believed that this transformation annot be done completely automatically [PATERNO et al., 1997a].

This work resulted in an algorithm for transforming tasks to interactors. The algorithm identifies the interactors, identifies the connections among them, and makes the interactor comply with the temporal relationships defined at the task level. 


\subsubsection{ADEPT}

To help designers refine their collective knowledge about tasks, a series of modeling tools have been developed as part of an Advanced Design Environment for Prototyping with Task Models - ADEPT JoHNSon et al., 1993, WILson et al., 1992, WiLson et al. 1993]. These modeling rools provide editors, browsers, and libraries, which case the process of oryanizing the vast amount of information about the task.

The process of producing the computer version of the task models commences when the designers have constructed and agreed on the pencil-and-paper versions. The shift from paper to computer-based representations may come earlier or later depending upon how easily the designers and end-users feet that the different media of representation can allow them to develop their joint understanding of the tasks and the design problems.

During the end-users' and designers' development of the rask models, it will become apparent that numerous task models will be required. There will be one task model for each ansk and there may also be an overview model, which shows how the different tasks are related. Editors allow the end-users and designers to rename, delete or replace an existing entry, add a procedure or a subgoal to the task model, and expand or contract the model in depth. It a further stage of development, objects can be edited and their properties changed. The objects are domain objects that are affected by or used in the particular task defined by the currendy displayed task model. 
The task modeling acrivity proceeds by developing the detail of the various tasks of the endusers to produce a series of task models represented in the task model library of the ADEPT toolsct.

At this point, the end-user and the designer agree that it would be appropriate to begin to develop some design ideats and see how they fit into the activities.

The end-users are able to understand and contribute to the design discussions because the focus is on activities, tasks, and objects associated with taking end-user's actuvines. Consequently, as the design ideas progress, the designers and end-users return to the IDEPT tools and begin to transform the task model they have constructed into a design.

The designer begins to create a new version of the ask model, which is called the "design task model." The design task model is a high-level specification of what the design wall allow endusers 60 do. Thus, it is the first attempt at meeting the design requirements by saying what the computer system will enable end-users to do and how it will affect their work. From the design task model the designers and end-users can produce an Abstract Interface Model ( $\mathrm{X} M \mathrm{M}$ ) using the ADEPT tools.

The AIM is a model of the interaction aspects of the proposed design. It takes the structure of the tasks as described in the design task model and instantiates that as the structure for the interaction dialog. The AM provides a use-iase model of the way the various objects of the design will be linked together and used during a particular interaction scenario. The object component of the AIM provides a complete description of all the objects necessary to support 
the designed dialogs and to allow the designed tasks to be carried out. This is a textual description of the objects, their properries and links to other objects, including dialog links.

The AIM is developed further to produce a Concrete Interface Mlodel (CIM), which provides a runnable version of an actual interface with which the end-users and the designers can interact. The method by wheh the CIM is developed is through refining the design to detinl how the interaction described in the AIM is to be realized in an actual computer interfice.

\subsubsection{Viewpoints}

This work describes an approach to the "multiple perspective" problem in sotinare engineering - many actors, many representation schemes, diverse domain knowledge and different development strategies, all in the context of disturbed asynchronous development.

Tradional approaches to the problems of distributed work use a central databasc, or repository, to which all team members have communicaton access. Such centralized approaches do not adeyuately support the reality of distributed engineering (Co This)sis et al., [993].

The alternative, a fully decentralized environment, is problematic because of the difficulties of maintaining the consistency benveen a large collection of agents. However, these problems can be overcome by recognizing that maintaining global consistency at all times is an unnecessary burden. That will maximize design freedom, and prevent premature commiment to design decisions. The focus therefore shifts from maintaining consistency to the management of inconsistencies. 
The framework upon which this work is based supports distributed software engineering in which multiple perspectives are maintained separately as distributed objects, called l'tewpoint. [FINIELLSTEIN et al., 1992]. A ViewPoint can be thought of as a combination of the iden of an 'actor', 'knowledge source', 'role' or 'agent' in the development process, and the idea of a 'view' or perspective that an actor maintains.

Relationships berween ViewPoints are expressed by a set of Consistenty Rules |E.LTERRR(x) et al., 1994|. The resolution process is concerned with establishing a relationship between rwo ViewPoints. Resolution only becomes necessary if consistency check failed, ant the VicwPoint owner wishes to correct this. In many cases, resolution will not be necessary after the tailure of a rule, because the inconsistency can be tolerated.

The goal of inconsistency resolution is to (re-)establish the reladonships contained in the rule or rules which failed. If a relationship did previously hold, information about subsequent changes can be used to guide the resolution process. A prototype computer-based environment and associated tools (the l'iever) have been constructed to support this framework [NI'SEIBEH :ND FINKELSTEIN, 1992].

\subsubsection{Summary}

Transforming UiN descriptions to Petri nets (section 2.3.1) is a transition from the behavioral domain (represented by the LAN) to the constructional domain (represented by Petri nets). This transformation tightened the formalism of the UAN and increased the ability of processing it automatically. Unfortunately, this approach focused only on one aspect in the 
UAN model which is the user uttions column specifications. Further work is still needed to transform the entire UAN table (i.e., all its columns) to Petri ners.

Paterno's team (section 2.3.2) succeeded in making the transition from the behavioral domain (ConcurTaskTres) to the implementation side (sofrware architecture). This work can be used to both design and develop new systems but mught not help to analyze exusting ones. I.e., existing systems (represented by their design and implementauion models) never stop evolving, leading to the need for a mechanism by which we can link existing models as they cocrolve. That is our objective in this thesis as we aim to generate links benveen design artifacts which map one arritact to another.

The ADEPT tools (section 2.3.3) allow designers to start with a TKS task model, and go through several transformations until producing a final runnable model of the interface. Then, the result is a complete system. The disadvantages of this approach is that these tools are strictly dependent on the task model used (TKS); the final product is too constructional (not an architecture); and it is a one-way transformation, i.e., from the rask model to the construction of the user interface.

Lastly, an important advantage of the ViewPoints approach is that it more accurately reflects actual work practices. Tolerance of inconsistency allows actions affecting more that one ViewPoint to be de-coupled. This facilitates distributed work by allowing responsibility to be devoted to individual ViewPoints. 
We aim by our approach in this thesis to semi-automatically generate links between the L'AN task model and the Clock architecture. We expect that the semi-automation approach will encourage designers to generate the links between the artifacts whenever they evolve.

The next chapter gives more details about the LAN and the Clock architecture models, and introciuces the I iska tool which is used to visually browse the links benveen them. 


$$
\text { Chapter } 3
$$

\section{DESIGN ARTIFACTS BROWSED IN VISTA}

The work done in the l'istu tool [BRoWN et al., 1998| motives the research presented in this thesis. Vista showed that design arrifacts developed to produce user-interfaces can represent radically different views of a system under development. The Vista toul was developed to help designers explore design artifacts through visual links relating these artifacts. Vista reyures developers to manually specify links between design artifacts, motivating future research of investigating how links can be created and maintained in a more automatic manner. This thests presents Adligo, a tool that semi-automatically generates links benveen design artificts, represented in a form suitable for viewing in Vista

In this chapter we explain the different design artifacts supported by Vista, and show how they are linked together visually. We support our discussion by examples drawn from the Ciritial Patb Method (CPM) Planner application [GRAHAM, 1997], which was developed independently of this project.

The chapter is organized as follows. Section 3.1 introduces the notion of ask modeling. Section 3.2 describes the interface design of the example application, the CPM Planner. Section 3.3 gives a practical introduction for writing a task-oriented specification using the UAN notation. Section $3 .+$ describes the Clock architecture and some of its features. Section 
3.5 presents the Vista tool and describes how it visually shows the links relating the L'AN and the Clock architecture model.

\subsection{Task Modeling: Task Hierarchy for Project Planning}

I.et us imagine that we are in the process of developing a sysem for project management. ()ne of the methods used for this purpose is the Cridical Path Method (CPMD). Lising this method. projects are decomposed into job steps. Dependencies among job steps are alssigned by arranging them into a network (figure 3.2). Resources are allocated to the job steps. Fnd-users maly reallocate resources upon identifying the critical path in the network /GR.IHLWI ct al.. 1996b]. 'To describe how to manage a project, designers can use a tast model. A task model is a design artifact resulting from the task analysis process [BERRY et al, 1997. DIAPER, 1989, J()HNSON at al., 1995].

I task model record: the tasks that potentiai end-users of the system may need to pertiorm to do their jobs, independent of dealing with a particular computer system. The design of an interactive system is based on supporting these tasks. Figure 3.1 shows the task hierarchy for the CPM Planner application. It describes how to carry out the task of planning a project. This figure shows how to decompose the main goal into subtasks that help achicve this gral. The root task of planning a project is split into the two subtasks of specifying the steps involved in the project and how they depend on each other, and of allocating resources to each step (This task is not supported by the program of figure 3.2). The task of specifying job steps and dependencies is solved via the task of creating a CPM network, which in turn consists of the 


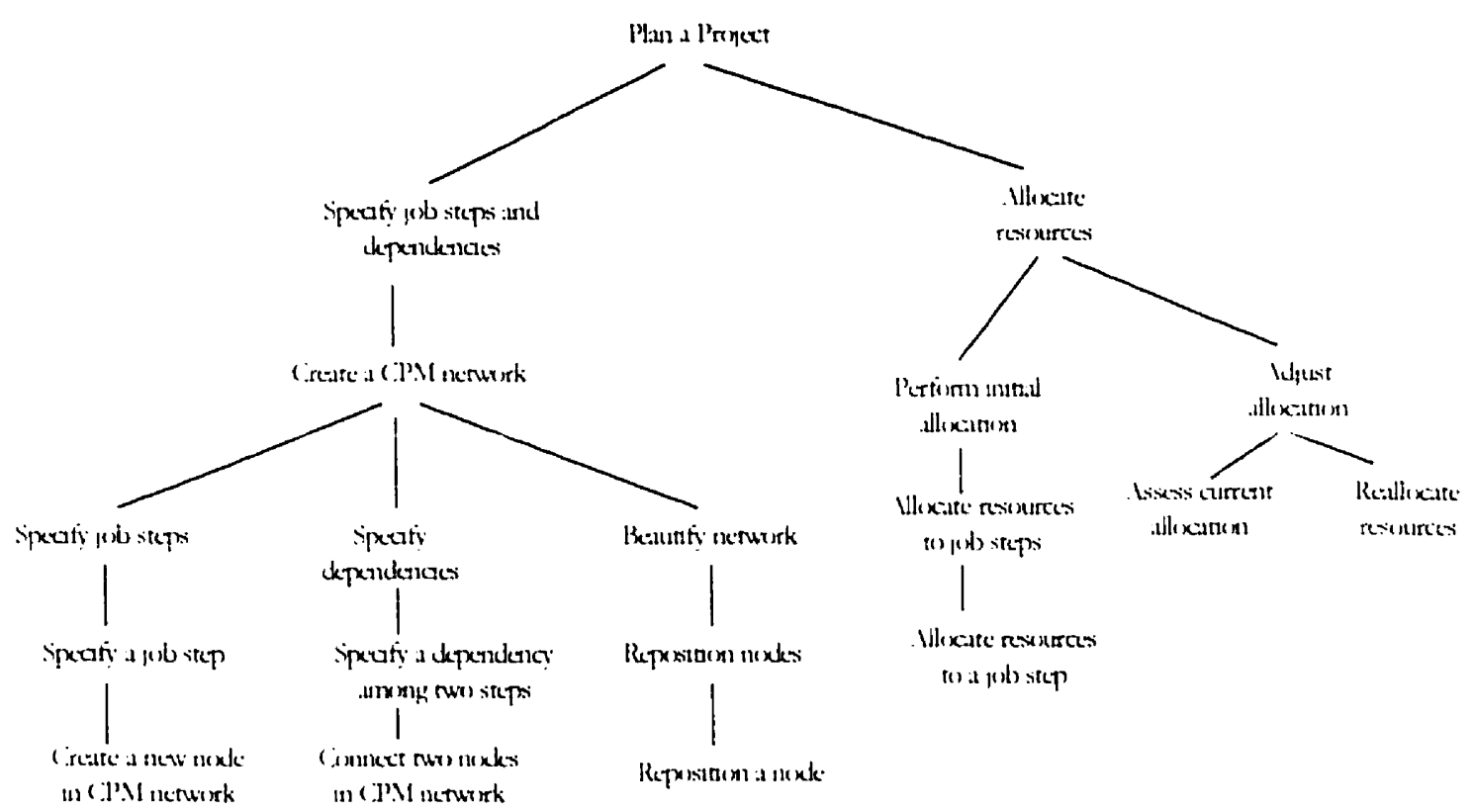

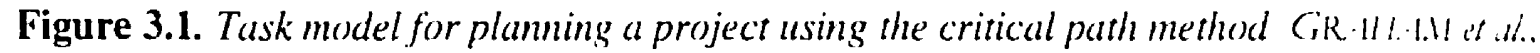
1996.2 .

subtasks of specifying the job steps, specifying their dependencies, and beautifying the presentation of the network.

\subsection{User Interface Design: The CPM Planner Application}

The previous section discussed the end-user tasks needed to plan a project. Figure 3.2 shows the user interface of the CPM Planner application, a groupware project management tool developed in the Clock language [GRAHAM AND URNES, 1996]. The CPM Planner allows participants to create nodes in a network. Nodes are connected by drawing a line from a source node to a destination node. Each node is assigned a sequential number. A control panel contains buttons for switching the mode of operation: repositioning, creating and connecting nodes. 


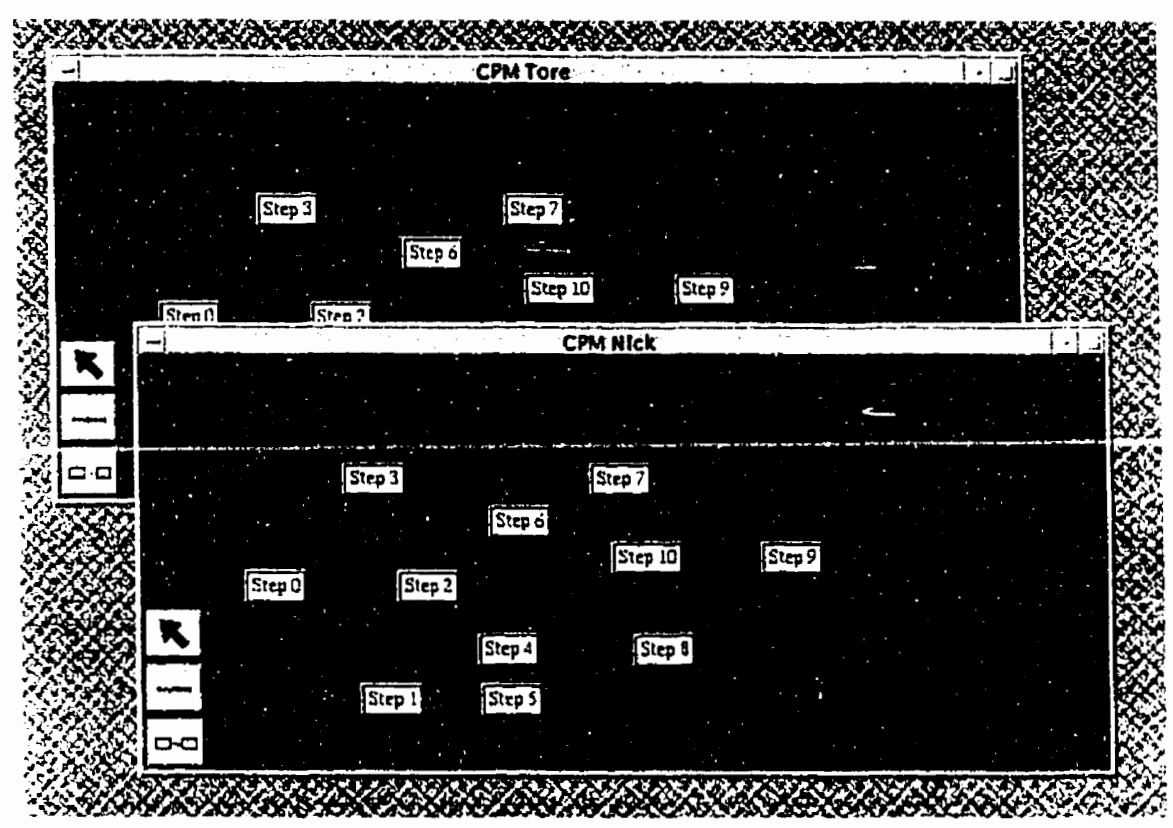

Figure 3.2. A collaborative tool (the (PM Planner). Tore and Nick are asing this appliation. They can connect, reporition. and add nodes. Eath user ian see the other's display GR HIt.11l et al., 1996b:

The next section describes how we can use the $\mathrm{LAN}$ notation to write an accurate specification for such an interface.

\subsection{Task-Oriented Specification Using the UAN}

A task-oriented specification encodes how tasks are carried out with the user interface, from the point of view of the end-user. HCI designers use the UAN to encode their tasks in a tabular format.

Section 2.1.1 gave a brief description of the UAN. This section is a practical [H.ARTSON et al., 1990] rather than a theoretical introduction [SIOCHI AND HARTSON, 1989]. We first introduce the UAN through a simple example, then proceed to more complex examples illustrating the UAN's advanced features. 


\subsubsection{A Simple Example}

Suppose that we want to describe a simple task such as selecting a mode of operation (e.g., repositioning, connecting, or adding nodes) in the CPM Planner application. In prose, this task can be described as follows:

(1) Move the pointer to the desired mode button.

(2) Depress and immediately release the mouse button.

The user action portion of the LIN description would be:

$$
\begin{aligned}
& \text { (1) } \sim[\text { mode } \mid \\
& \text { (2) } M v^{\wedge}
\end{aligned}
$$

The - denotes moving the pointer, in this example into the context of the mode button. The second line represents depressing (v) and releasing $\left(^{\wedge}\right)$ the mouse button ( $\left.\mathrm{M}\right)$.

Let us consider another example: the task of moving a node. We can state this in prose:

(1) Move the pointer to that node. Depress and hold down the mouse button. By depressing the mouse button, the node is considered to be selected and accordingly its display is highlighted. 
(2) While holding down the mouse button, move the pointer.

(3) Release the mouse button. The display of the node is now moved to the locarion where you released the button.

The user action portion in the UAN can describe this task as follows:
(i) $\sim$ node $\mid \mathrm{Mv}$
(2) $\sim[x, y]$
(3) $M^{\wedge}$

The tirst line means that we move the pointer into the context of the node and clepress the mouse button. In the second line, $\sim[x, y]$ indicates movement of the pointer to an arbitrary point $x, y$ in the network. The mouse button is released in the last line.

\subsubsection{Interface Feedback}

The previous section showed how to describe the user actons necessary for a rask. If we compare the LAN description with the prose in the simple example discussed above, we find that the prose contains feedback and semantic information as well as the user actions. The interfice feedback information represents the responses of the user interface to the actions carried out by the end-user.

Figure 3.3 shows a UAN table, which is read left to right, top to bottom. It indicates that when the end-user moves the pointer to the node and depresses the mouse button, the icon is highlighted (node!). As the end-user moves the pointer about the network, the highlighted node is displayed at that location. Finally, upon the release of the mouse button, the node is 


\begin{tabular}{|l|l|}
\hline TASK: Move a node \\
\hline USER ACTIONS & INTERFACE FEEDBACK \\
\hline$-[$ node] $M v$ & node! \\
\hline$-[x, y]$ & Display node! at $x, y$ \\
\hline$M \Lambda$ & Display node at $x, y$ \\
\hline
\end{tabular}

Figure 3.3. UAN description of the task "move a node" with interface fectback.

\begin{tabular}{|l|l|}
\hline TASK: Select a mode & \\
\hline USER ACTIONS & INTERFACE FEEDBACK \\
\hline$\sim$ mode] Mv & $\begin{array}{l}\text { mode-! ! mode! } \\
\forall \text { mode' ! : mode' -! }\end{array}$ \\
\hline$M^{\Lambda}$ & \\
\hline
\end{tabular}

Figure 3.4. UAN description of the task "select a mode" (more precise" feedback description.)

\begin{tabular}{|l|l|l|}
\hline \multicolumn{2}{l}{ TASK: Select a mode } \\
\hline USER ACTIONS & INTERFACE FEEDBACK & INTERFACE STATE \\
\hline$\sim$ [mode] Mv & $\begin{array}{l}\text { mode-! : mode! } \\
\forall \text { mode'! : mode-! }\end{array}$ & selected = mode \\
\hline$M^{\wedge}$ & & \\
\hline
\end{tabular}

Figure 3.5. UAN description for the sask "select a mode" with the interface state information.

displayed in the new position. Note the line-by-line associacion of feedback with the corresponding user actions. This level of precision is lost in the prose description, in which actions and feedback are mixed together. For instance, consider the rask of selecting a mode:

(1) Move the pointer to the desired mode button.

(2) Click the mouse button and the button will be highlighted.

The UAN description for this task is shown in figure 3.4. The feedback means that whenever you select a mode button, all others are deselected. A highlighting action is applied to the icon 
(mode!) only if the icon is not already highlighted; $\forall$ means "for all", and a colon is used between the condition and the corresponding feedback.

\subsubsection{Interface State}

It may be necessary to include some state information both for the interface and for its commetion to compiltution, associated with user actions. Figure 3.5 shows the interface state of the task "select a node". The variable select is set to the new selected mode. As another example, figure 3.6 shows that depressing the mouse button when the pointer is over an ubject icon causes the object represented by that icon to be selected. If HCI designers are concerned about the location of the icon as a part of the computational component of the application, rhey can add the connettion to computation column as shown in figure 3.6.

\subsubsection{Context of Objects}

The symbol $\sim \mathrm{X}]$ describes the end-user task of moving the cursor into the content of the interface object $\mathrm{X}$. Moving out of the context of the same object is denoted by $|\mathrm{X}| \sim$. The context of an object is the visual representation of the object on the display. For example, in the expression $\sim[\mathrm{X}] \mathrm{Mv}$, it is assumed that the pressing of the mouse button takes place within the context of $\mathrm{X}$.

\subsubsection{Summary}

Section 3.3 gave an introduction of how to write a task-oriented specification using the $L A N$ through some examples. The UAN descriptions are written in tables. A table may consist of 


\begin{tabular}{|l|l|l|l|}
\hline TASK: Move an object icon & $\begin{array}{l}\text { INTERFACE } \\
\text { STATE }\end{array}$ & $\begin{array}{l}\text { CONNECTION TO } \\
\text { COMPUTATION }\end{array}$ \\
\hline U [object_icon] MV & $\begin{array}{l}\text { object_icon-! : } \\
\text { object_icon! } \\
\forall \text { object_icon'! } \\
\text { object_icon'-! }\end{array}$ & selected = object & \\
\hline & & $\begin{array}{l}\text { location (object_icon) } \\
=(x, y)\end{array}$ \\
\hline
\end{tabular}

Figure 3.6. LAN description of the task "move an object icon" with connection to compuration information.

wer ations, interiace feedbate, interfice state and ionnection to computation columns. The LAN notarion describes how the interface display and state respond to the user actions. The "- fimtent" denores changing the context in which user actions take place. Identifying the L $I N$ context is one of the key ideas used to generate the links semi-automatically as we will see in chapter $t$.

\subsection{Building the CPM Planner Using the Clock Architecture Style}

Software engineers decompose complex systerns into small parts using software architectures. This reduces the complexity of implementation, evolution and maintenance of the system [GR.THAM AND L'RNES, 1996].

Clock architecture style is an extension of the Model-View-Controller (MVC) paradigm [KRISNER AND POPE, 1988] (sce section 2.2.1). MVC was designed to support the evolutionary design of architectures for interactive, multi-user sy-ems. The Clock architecture style provides the properties of restricted scoping to reduce direct dependencies among components, automatic message routing via delegation, and easy modification of the 


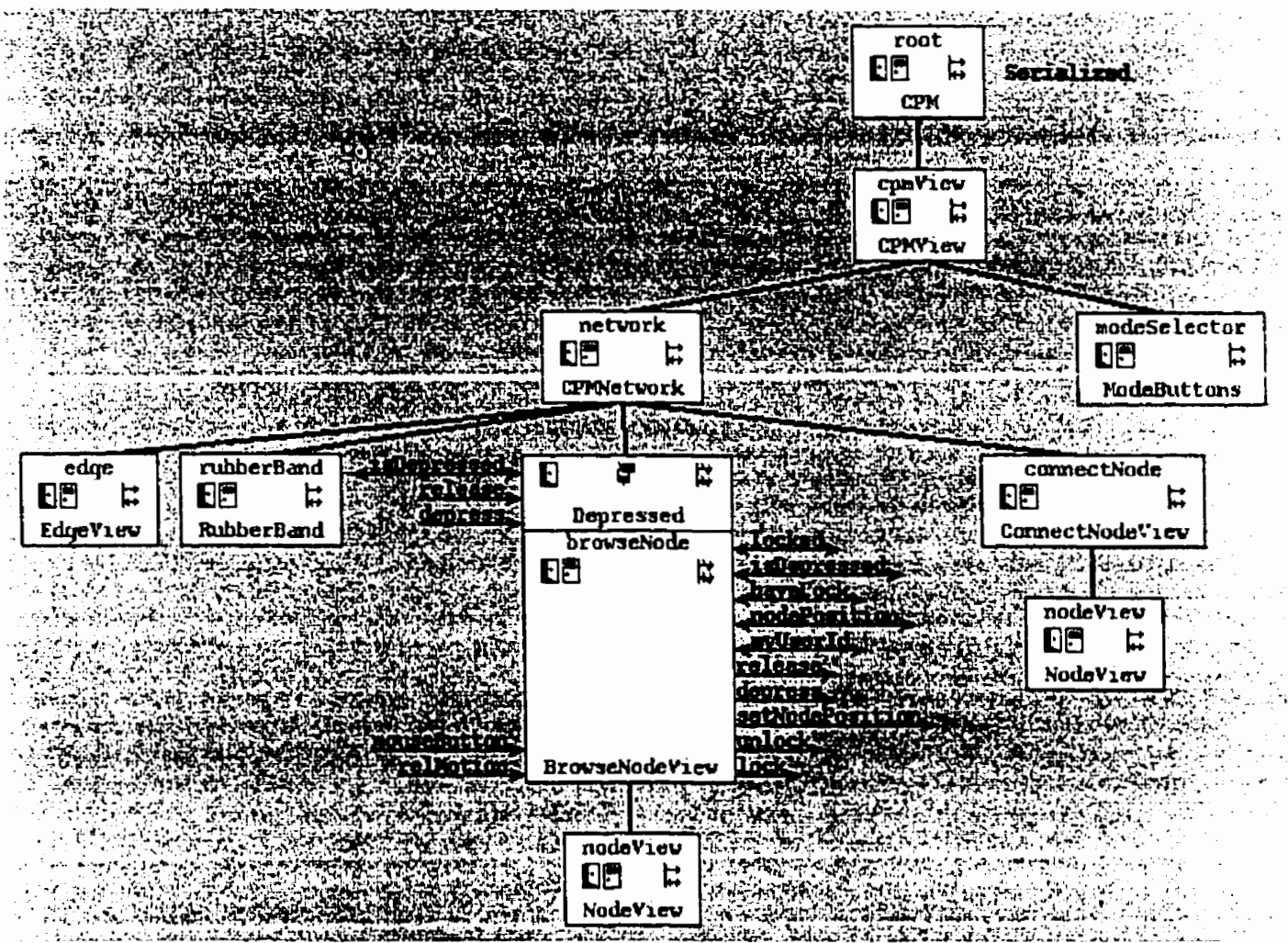

Figure 3.7. The CPM Planner Clock architecutre. The Clock architecture is a tree of communicating components. The Depressed ADT is attached to the browseNode component.

hierarchical structure of architectures [GRAHAN AND URNES, 1996]. Clock is supported by the visual ClocklW'orks programming environment [GRAHAM et al., 1996a].

Figure 3.7 shows that the Clock architecture is a tree of communicating components. Components may process end-user inputs or inputs from other components. They may also produce graphical output on the display. Components can be augmented by abstract data types (ADTs), such as the Depressed ADT, which is attached to the browseNode component. Each component has a name (e.g., browseNode) and a class (e.g., BrowseNodel'iew). 
Components may be grouped. Clicking a component's open door icon opens the group and reveals its intemal structure. The close door icon elides detail. The interfice of a component can be shown by toggling the component's interface icon. The imput, request and update messages allow the components to communicare. For example, baveLoik and isDepressed are requests; set NodePosition and release are updates; mouseButton and relMotion are input events (see figure 3.7). The arrows on the lett side of the component indicate the messages the component may receive, while the arrows on the right side indicate the messages the component may issue. The method may be a request or an update. A bandler is a method belonging to a specific component or an ADT.

Messages are automatically routed up the tree to the nearest component capable of handling them. This means that components can only access the data of components appearing above them - components may not directly communicate with their children or siblings [GRAHAM et al., $1996 \mathrm{~b}\}$.

The next sections describe two signification features in this architectural model constraints and automatic routing.

\subsubsection{Communication via Constraints}

Restrictive scoping rules are imposed in the Clock architecture. Therefore, the ways in which components can directly communicate are reduced. In order to allow components to indirectly communicate, Clock embeds a constraint mechanism into its architecture language. These restrictions have the positive effect of reducing the number of direct dependencies among components, in turn reducing the impact of architectural change [GRAHAM et al., 1996b]. 


\subsubsection{Automatic Routing}

As the architecture evolves, it is common to add ADT's, split or combine components, and othervise change the locations of where updates and requests are handled. In typical architecture descriprions, communication is bound to specific components; i.e., it is necessary to specify exactly where a method call is directed. This form of explicit targeting makes code less robust to change, since as components evolve, the method may no longer be handled by the same component. In Clock, requests and updates are automatically routed to the nearest component above the issuer of the message that is capable of handling it. This routung mechanism is only possible due to Clock's restricted visibility rules, which are in turn made possible by the use of constraints [GR:HH.LI et al., 1996b].

\subsubsection{Summary}

Secrion $3 .+$ introduced the second design artifact which is the Clock architecture model. The Clock architecture is a tree of communicating components. Components may be augmented by ADT's. Requests and updates are the interfaces of components and ADT's. Components can communicate via constraints. Such constraints create restricted visibility rules that make the automatic routing mechanism possible in this architecture style.

\subsection{The Vista Tool}

['ist is a prototype tool developed for examining the links between design artifacts [BRONN et al., 1998]. It allows the designers to simultaneously navigate hyperlinked task analysis, taskoriented specification, software architecture and code documents. We describe the features of 
this tool through the CPM Planner. Vista uses the Clock architecture style for developing user interfaces [GRAHAN AND URNES, 1997].

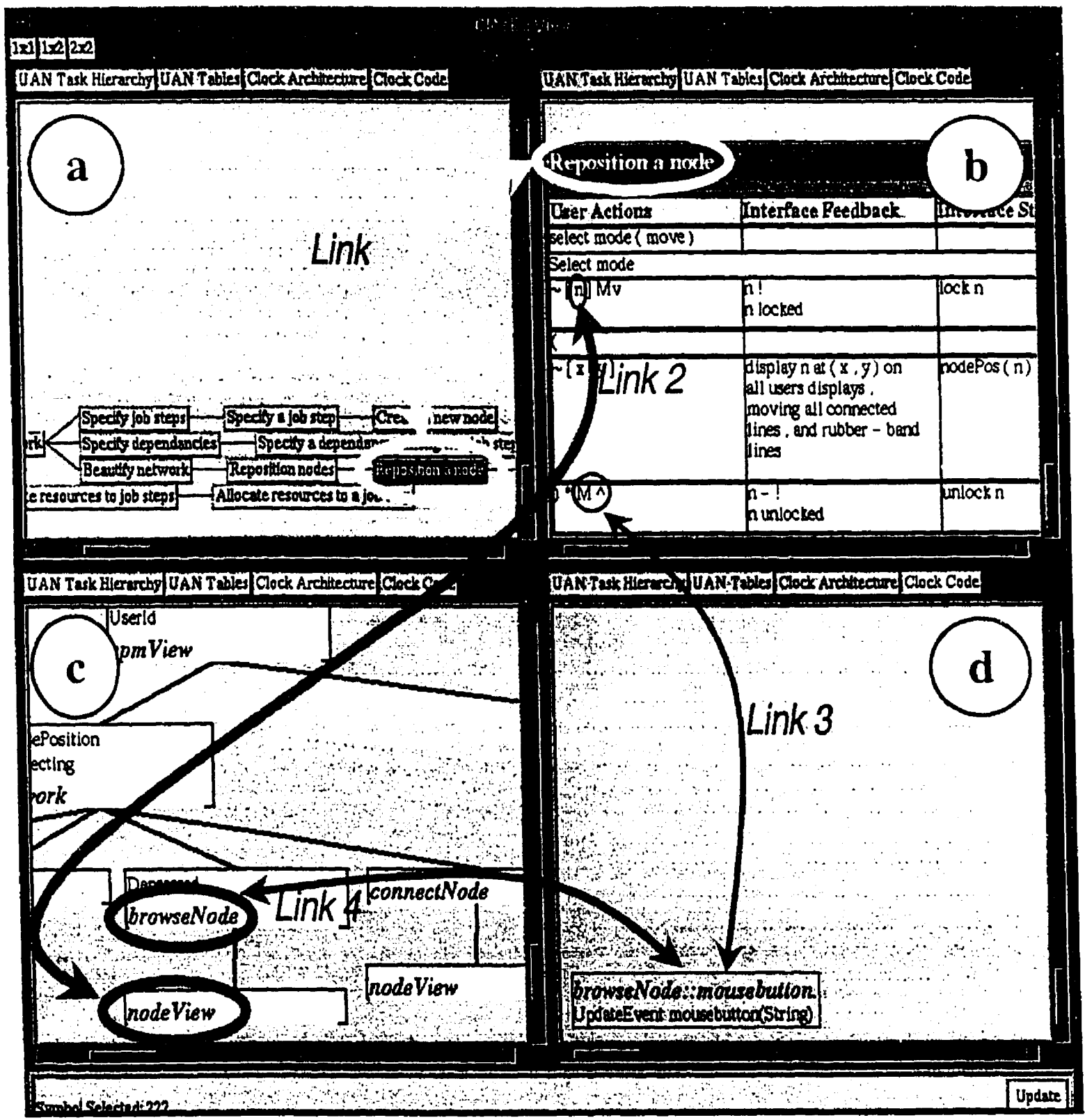

Figure 3.8. Linked views in Vista. A designer has selected four views of the CPM Planner application: (a) the task hierarchy: (b) the task-oriented specification in the UAN; (c) the Clock architecture of the application, and $(d)$ the Clock code. The Vista display has been manually annotated to show some of the links between the design representations. 


\subsubsection{Vista Puts Them All Together}

Figure 3.8 shows four views of the CPM Planner as displayed in the l'istu tool. The four panels are (a) the hierarchical cask model, (b) the L.AN task-oriented specification, (c) the Clock architecture and (d) the Clock code. These views show the design and implementanion of the task "Reposition a Node". The views of these four panels are connected by hynerlinks, so that when designers click on information in one panel, the corresponding information is shown in the others. Figure 3.8 has been annotated to show four of the hyperlinks available to designers. For example, clicking on the "Reposition a Node" task in panel (a) pops up the corresponding $\mathrm{UAN}$ table in panel (b) (link 1 in figure 3.8). Designers may the the Vista display into one, two or four panels, allowing them to select between more views and bigger panels.

The next section describes the links between the task-oriented UAN specification and the system-centered Clock architecture specification. Links between other specifications are not as complex. The section explores how the UAN concepts elements context, wser attion, interjate ferdback and interjace state are linked to the architectural elements of components, input etrents, inew methods and $-1 D T$ 's.

\subsubsection{Linking the UAN Contexts to Architecture Components}

Clock architecture components implement the UAN contexts [BROWN et al., 1998]. For example, in the task "Reposition a Node" of Eigure 3.8, the UAN context $"$ is implemented by the nodel'iew architecture component. In Vista, clicking on the $n$ in the UAN table highlights the nodel iew component in the architecture view. 


\section{[101]120]200}

\section{Coch : Vis}

UAN Task Hilerarchy UAN Tables Clock Archittecture Clock Code.

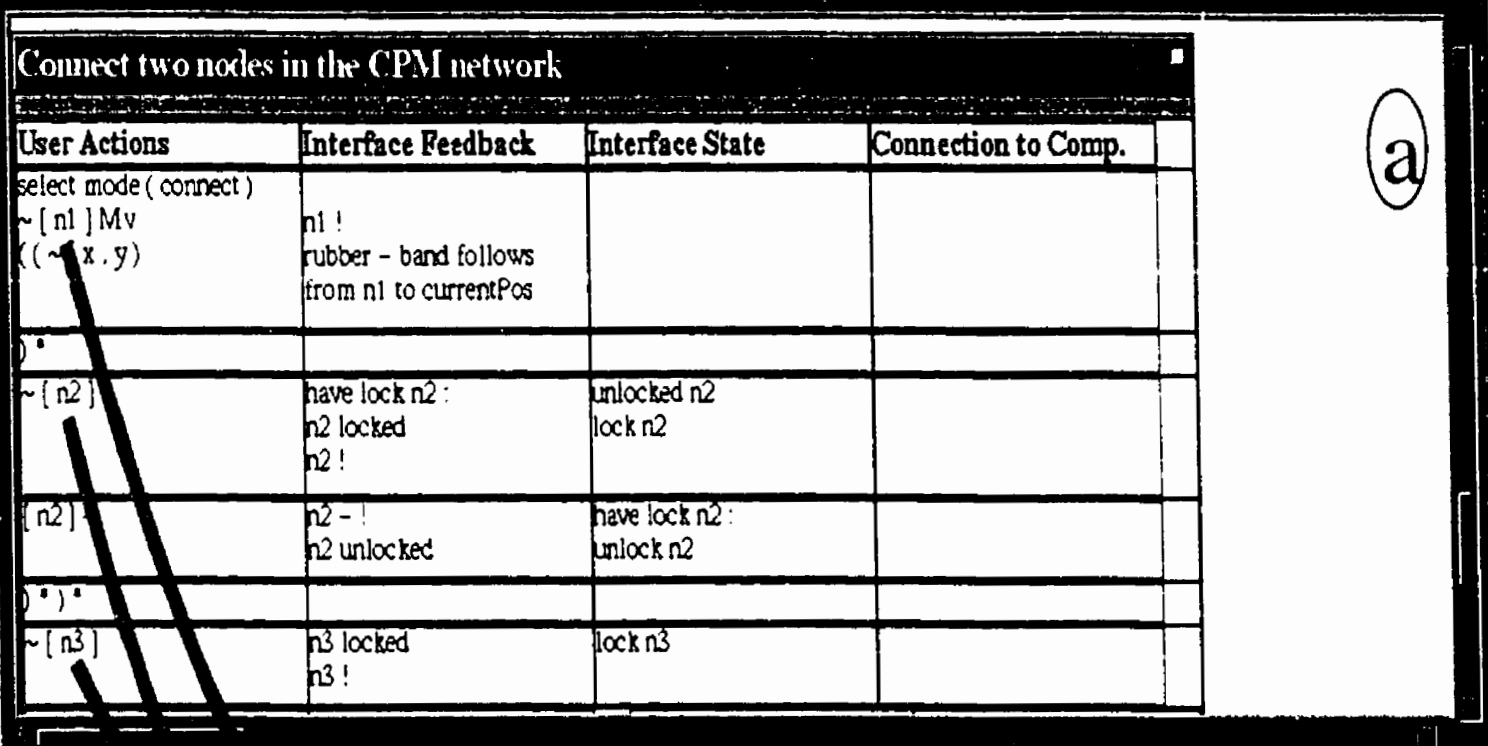

UAN Task, era by U, TTables Clock Architecture Clock Code

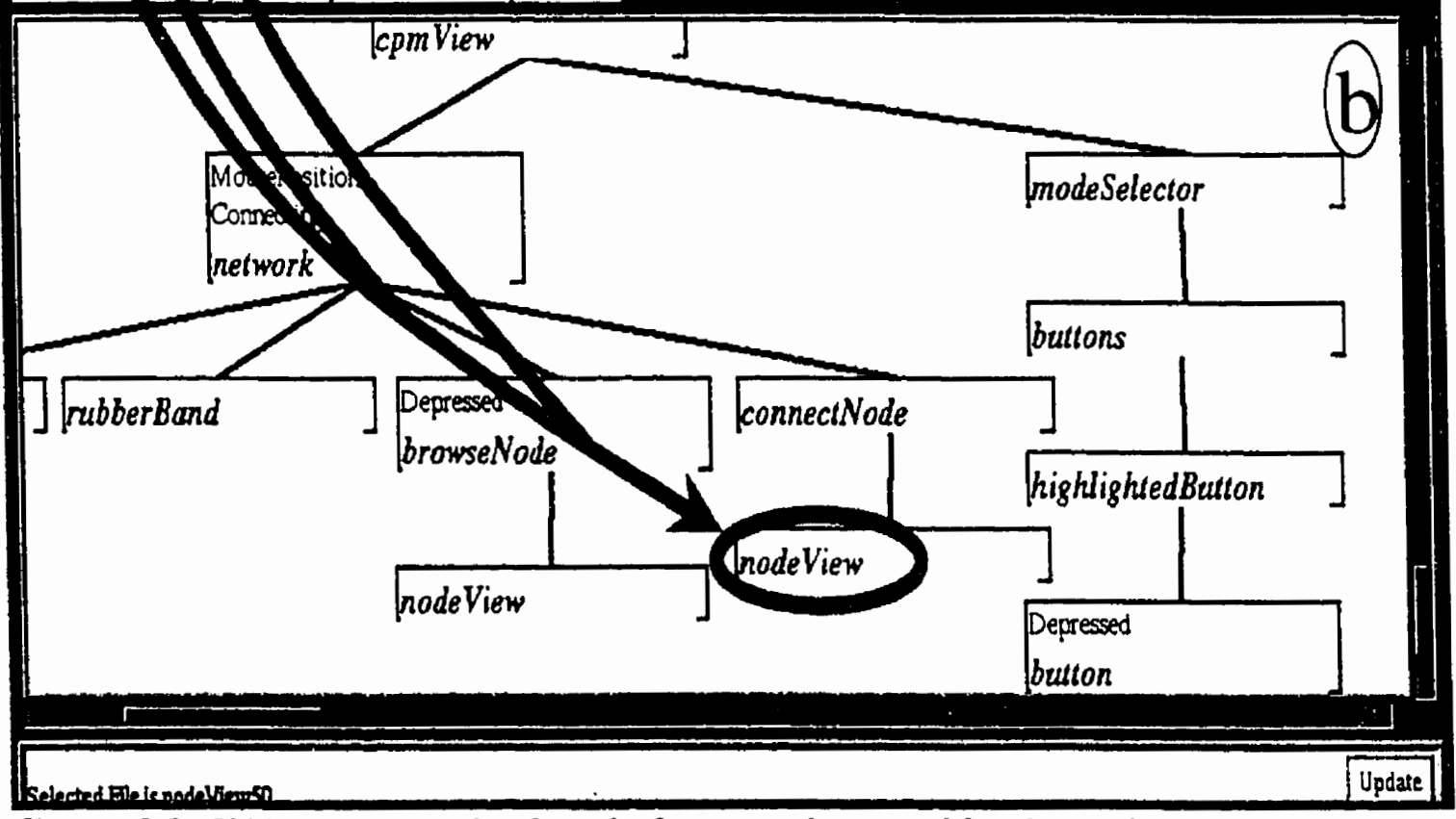

Figure 3.9. UAN contexts $n 1, n 2$ and $n 3$ are implemented by the architecture component "nodeView." 
Identifying which architecture component implements each UAN context is non-trivial [BROWN et al., 1998]. The HCI designer and the software engineer may have given the same entity a different name (as in the example above). Another problem is that the relationship between the UAN contexts and architecture components is not one-to-one. Architecture designers do not necessarily create a new component whenever the LAN splecification identifies a new context. Figure 3.9 shows how one architecture component can implement more than one UAN context. The UAN contexts $n 1, n 2$ and $n 3$ are implemented by the nodel'iew component. Providing a solution for such problems is the core of this thesis (sec chapter 4: Semi-Automated Link Generation Process.)

\subsubsection{Linking the UAN Actions to Architecture Components}

In the UAN descriptions, user actions specify what inputs end-users perform in order to perform their task. User actions occur within contexts. For example, in order to move a node to another location (after switching to the repositioning mode), the end-user positions his or her pointer over that node $(\sim[\mathrm{n}])$ in the nodel tew view and then depresses the mouse button (MIv). Therefore, the action "Mv" occurs in the context of nodel 'iev. L'ser actions are processed by the architecture components implementing the action context. Since nodel 'iew implements the context $n$, it also processes the user action " $\mathrm{Mv}$ " in the method mouseButton, whose prototype is shown in figure 3.8(d).

Accordingly, user actions are linked to the components that implement those actions. Clicking on the "Mv" symbol in figure 3.8(b) displays the method that handles mouse button events (link 3), and highlights the component that contains the method (link 4). 


\subsubsection{Linking the UAN Interface Feedback Specifications to Component}

\section{Views}

The interface jeedback column in a UAN table specifies how the display should change in response to end-user input. The software engineer uses this information to implement the ritev mcthods of architecture components. In the CPM planner, clicking the " $n$ !" or " $n-$ !" text in figure 3.8(a) highlights the nodel tiew component of figure $3.8(\mathrm{~b})$ which implements the appearance of the node.

\subsubsection{Linking the UAN User Interface State to ADT's}

User actions can cause displays to change but they can also trigger state changes. In the LiN, these state changes are specified using pseudo-code in the Interficte-stute column of the L'AN table. In Clock architectures, states are implemented as abstract data types (AD'T"s), which are attached to architecture components. The state that is referenced in the pseudo-code of the U.AN tables can then be linked to the AD'T's implementing the state.

For example, in figure 3.8(b), the UAN specifies that clicking on onc of the nodes (after switching to the repositioning mode) should lock that node "Lock $n$ ". As the end-user moves the node around the screen, the node position is updated accordingly "nodePosition(n): $(x$, y)". Once the end-user rcleases the mouse button, the locking is rcleased "unlock n". The locking/unlocking mechanism is implemented using the Lock ADT, which is artached to the root component (not shown in figure 3.8). Information about the node position in the network is stored in the NodePositions ADT, which is also attached to the rot component. L'ista displays the links between interface state and ADT"s. For example, clicking on the "Lock n" or "unlock 
$\mathrm{n}$ " texts in figure 3.8(b) highlights the Lock ADT while clicking on the "nodePosition $(\mathrm{n}):=(\mathrm{x}$, y)" text highlights the NodePositions ADT.

\subsection{Summary}

The goal of this chapter was to describe the nvo design artifacts, the L'IN and the Clock architecture models, and show how to browse the hyperlinks relating them in the Vista tool. Most of the examples were derived from the CPM Planner application.

The chapter started by describing the task modeling and ask-oriented specifications using the UAN. It gave a simple pracrical introduction for the UAN notation and its tables. The Clock architecture was described as a tree of communicaning components. This archirecture styte is featured by the automatic routing mechanism, which is possible due to the Clock's restricted visibility rules. Lastly, we described the Vista tool, which is used to examine the hyperlinks relating the $\mathrm{LAN}$ and the Clock architecture models.

However, Vista. has some major problems. Links in Vista are hard-coded and manually specified. We may need to feed hundreds of links manually to Vista in order to relate the UAN and the Clock architecture models of a medium size case study (see chapter 6 : Experiments and Analysis). The problem gets more challenging if we wish to maintain these links as the design artifacts evolve. In this case, the link generation process is necessarily iterative in order to maintain the relationship between the two design artifacts. Chapters $t$ and 5 of this thesis describe how we provide a semi-automatic solution for such problems. 


$$
\text { Chaptert }
$$

\section{SEMI-AUTOMATED LINK GENERATION PROCESS:}

\section{THE ADLIGO TOOL}

In this chapter we introduce. Lliligo, a tool demonstrating the semi-automated process for linking two user interface design arrfacts: the UAN task model and the Clock archirecture. Adligo has built-in knowledge of the two artifacts to be linked. As shown in chapter 6, Experiments and Analysis, thus knowledge is not sufficient to derive a reasunably complete set of links. Therefore, a human familiar with the artifacts to be linked must provide additional informarion to enable. Adligo to produce a highly accurate ser of links.

We provide in this chapter a full description of the link generation process for potential users of Adligo. Implementation details are left for chapter 5 , while the tool evaluation is the subject of chaprer 6 .

The chapter is organized as follows. Section 4.1 presents the general framework of the link generation process. Section 4.2 presents a tutorial showing how Adligo is used to generate links. The tutorial shows how the links are derived for the different UAN columns, and introduces the dictionary and the pattern language supporting this process. Section 4.3 
discusses more details, such as the UAN context, subtask referencing, dictionary types, the order of the rules in the dictionary, and more details about the pattern language.

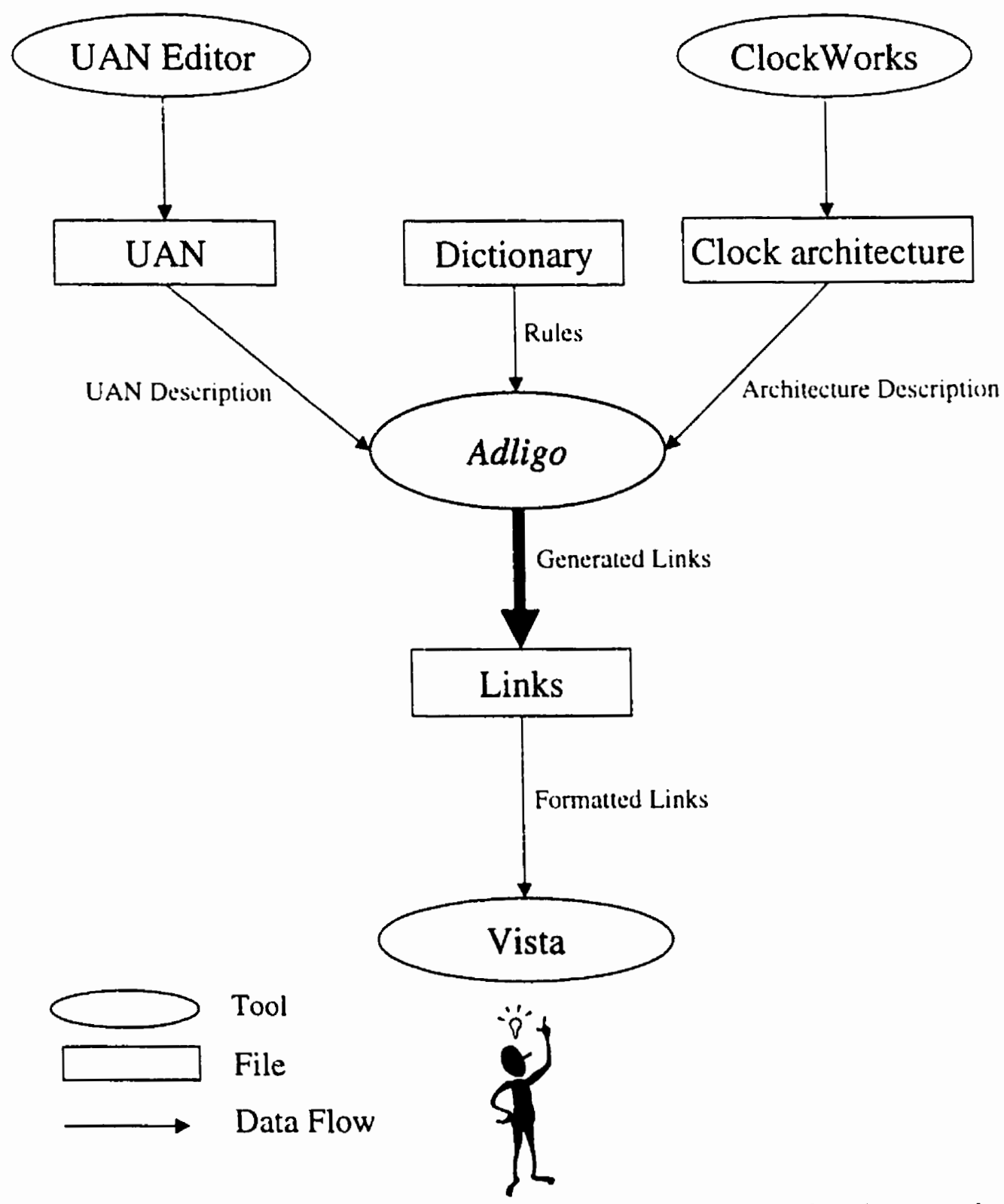

Figure 4.1. A frainework for generating links between task and architectural models in semi-automated fashion. 


\subsection{The Framework of the Link Generation Process}

Figure 4.1 shows the framework of the process of generating links berween the design and implementation of interactive systems. A UAN editor is used to write the task-oriented specifications, and to generate the LAN description as a text tile suitable for input to Adlig(). Since such an editor has not yet been implemented, this input file is currently prepared manually. The format of this file is described in appendix E. Programmers can write their programs in the Clock language using the Clocklt"orks visual programming environment [GRtHAlif et al., 1996a]. This rool writes the architecture of the application in a texrual format [Gr.1HAN, 1996] that can be fed into Adligo automatically. Finally, the designer provides a set of rules in a dictionary file. The dictionary is described in section +2.3 .

Adligo accepts those input files, analyzes the semantics of each design artiact (i.e. the LiN and the Clock architecture models) and derives links berween them. This process is carried out with the assistance of the dicrionary rules. The result of running Adligo is a set of links which connect the two notations. These links are written in text files which can be used as inpuc to the L'istu tool, allowing the links to be browsed graphically.

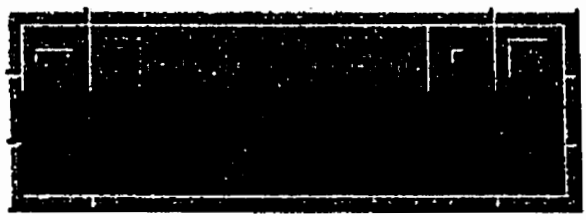

Figure 4.2. A simple Clock application, the Counter. A number is displayed in a window. This number is incremented when a user clicks it. 


\begin{tabular}{|l|l|l|}
\hline \multicolumn{2}{|l|}{ TASK Increment Number } \\
\hline USER ACTIONS & INTERFACE STATE & INTERFACE FEEDBACK \\
\hline$\sim[$ CountView] Mv & Count $+=1$ & Display Count \\
\hline$M^{\wedge}$ & & \\
\hline
\end{tabular}

Figure 4.3. The UAN description of the Counter application.

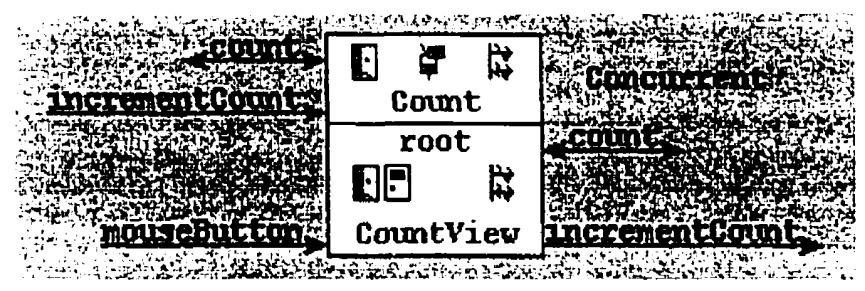

Figure 4.4. Clock architecture of the Counter application.

The tutorial in the next section uses a simple Counter application (figure +.2), which displars an integer number in a window; whenever the end-user clicks this number, it is incremented. Figure +3 shows a U:AN specification which records what actions a end-user must perform to increment the integer number. Figure t.t shows the Clock archirecture for the Counter application.

\subsection{Tutorial Introduction}

In order to illustrate the concepts and steps involved in the link generation process, we present a simple tutorial which shows how to link the UAN model and the Clock architecture for a small application like the Counter. Figure 4.5 shows the links which the HCI and software engineers expect to see between the UAN and the Clock architecture models of the Counter. The tutorial will show how we derived these links with Adligo. 
Throughout our discussion, we will be exposed to several concepts related to the link generation process. Section +.2.1 explains how we derived the links for the user actions column. Section 4.2.2 shows the importance of identifying the contexr component in links inference. Section +.2.3 introduces the dictionary as a transiation means. Section t.3.t introduces some new rerms related in searching the Clock architecture, stech as the sopte, the contest component and the cumrent siope. Section 4.2.5 shows the derivation of a link tor the tirst user action in the Counter application. Section +.2.6 shows that talligo has some built-in knowledge, which helps in the link derivation process. Section +.2.7 explains how to generate links for the U:IN description residing in the interface state column. Section +.2 .8 introduces the pattern language used in writing the diecionary rules. Section +.2 .9 explains how to generate links for the UAN description residing in the interfice feedbucte column. By the end of section 4.2.9, all the links for the Counter application are generated, and most of the concepts underlying Adligo are discussed.

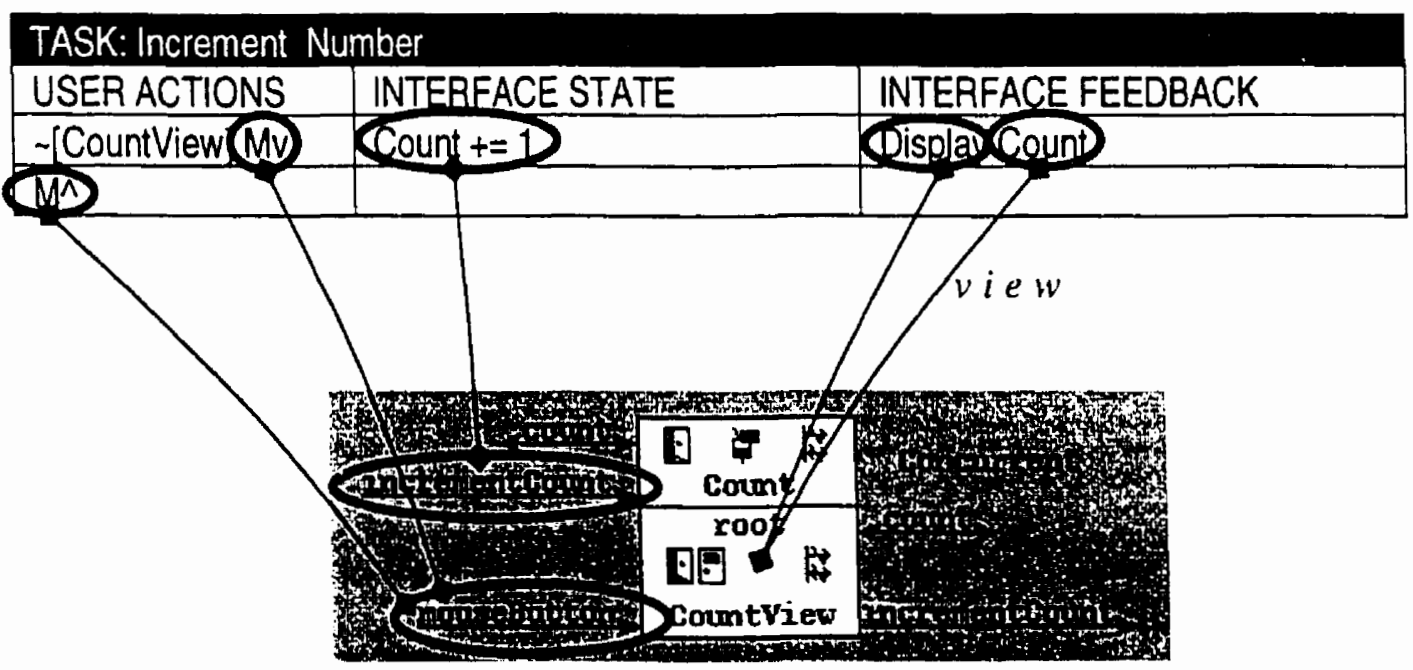

Figure 4.5. The generated links between the UAN and the Clock architecture of the Counter application. 


\begin{tabular}{|c|c|c|c|}
\hline \multirow[b]{2}{*}{ Rule\# } & \multicolumn{3}{|l|}{ DICTIONARY } \\
\hline & RULE SCOPE & UAN PATTERN & CLOCK ARCHITECTURE PATTERN \\
\hline 1 & & Mv & mouseButton \\
\hline 2 & & $M^{\Lambda}$ & mouseButton \\
\hline 3 & & $\$(\operatorname{var} 1)+=1$ & inc\$(var1) I increment\$(var1) \\
\hline
\end{tabular}

Figure 4.6. The dictionary used for the Counter application.

( )ur starting point in this tutorial will be from the UAN table shown in figure +.5 , trying to map every malits to its counterpart bandler in the Clock architecture. A malith can be any sequence of tokens that can be matched by a rule in the dictionary. A bandler is a method inside a component or an ADT in the Clock architecture. The process iterates over every table in the task model.

The navigation inside the $U A N$ table conforms to the direction of reading it: left to right, top to bottom. The Counter has only one task represented by one LIN table shown in figure t.3. In the following secrions, we will derive the links for this table.

\subsubsection{Linking the User Actions}

The first description in the user attions column is " $\sim$ CountView]", which means that the enduser moves his or her mouse pointer to the Counter view. From the other columns of the task description, we can see that nothing happens as a result of this action. We should not expect to find any handler in the architecture because that action does not need any particular manipulation. Nevertheless, this action is crucial because it changes the context in which the subsequent actions take place. This means that any future events (or actions) will be intercepted and handled by Count iew because it is the current context. 


\subsubsection{The Secret Is in the Context}

A context in the UAN description is the object to which the end-user moves. Changing the context is denoted by the UAN notation " $[$ context]". Any UAN context should be represented by a component in the Clock architecture. Therefore, we should search for a component named Comtl'iev. Since there is only one component in this hierarchy, the search process is trivial in this cxample (see figure +.t). From now on, we have to keep in mind that Our current context has been switched to Countl'tew.

The next action is "Mv", which means depressing the mouse button. In the Clock architecture, this event is handled by the Count "ien component via the predefined method mouseButton. "Thereby, we have found a link berween the UAN description "Mv" and the handler Countl'iev.mouseButton.

The analysis of the decision that has led to this association is as follows: Couml "een is the component that represents the current context of user actions. Accordingly, it should contain a method that handles the user action denoted by "Nv". We know (as humans) that the notation "Mlv" and " $\mathrm{M}^{\wedge}$ " mean a mouse button depressed and released respectively. Therefore, the correct method (in Clock) to handle such events is the predefined method mouseButton. Such human knowledge needs to be fed to the Adligo tool in order to generate such links.

\subsubsection{A Translator Is Needed: The Dictionary}

The need to supply Adligo with some human knowledge motivated the introduction of the dittionary. A dittionary is a set of rules that translates the UAN notations to Clock architecture symbols (figure 4.6). As shown, the dictionary is a simple table composed of three columns: 
mile siope, $L$ - IN pattern and Clock aribitecture pattem. The table has a set of rows or entries called nules.

The rule siope column determines the scope of application of that rule in the task model. The rule can be local (i.e., applicable only to a specific task), or global (i.e., applicable to all tasks). To flag a rule as local to certain task, the task name can be written in the rule siope slot of that rule. Leaving that slot blank makes that rule globally applicable to any task in the model.

The $L_{-}^{\prime}-1 V$ pattem column contains a $U: I N$ description pattern written in a simple pattern language. Section +3.8 describes this language in detail. During the process of generating the links, Adligo tries to find a match in the UAN description using this U.AN pattern.

If there is a match, the Clock unthiteiture pattem column will be considered. This column contains another pattern that describes a potental handler in the architecture. Adligo searches the Clock architecture for this handler and if it is found, a new link is created.

\subsubsection{Searching the Clock Architecture}

Once a handler has been identified as the potental target of a link, Adligo tries to find the first occurrence of that handler within the siope of the context component. The siope of a component is the chain of components that starts from that component and goes upward undil reaching the root of the architecture tree. The context component is the Clock architecture component that represents the UAN context. The current siope is the scope of the context iomponent. 


\subsubsection{Deriving the Link for "Mv"}

The next user action in the Increment Number task is "MIv". If we search the dictionary (sce figure 4.6) top to the bottom, we will find that rule \#1 helps us derive such a link because "Mv" matches the UAN partern in that rule. Consequently, we proceed to search the Clock architecture for the Clock pattern "mouseButton".

\subsubsection{Adligo Has Some Built-In Knowledge about the Models}

The pattern "mouseButton" does not fully specify a handler since it shows the required method but does not specify its container (i.e., a component or an ADT). Adligo does not require the fully qualified name (the method and its container) of the handler becausc it knowss that any user action must be handled by a component in the current scope. Accordingly, . Lelligo locates the mouseButton method in the Count 'iew component, because it is the first component in the current siope that has the mouse Button methed.

Figure +.5 shows the link between the U.AN nutation "Mv" and the Clock architecture handler Count l'iew.muaseButton.

\subsubsection{Linking the Interface State}

The next column is the interficte state column, in which the designer expresses the idea of incrementing the stored number by the pseudo-code "count $+=1$ ". This pseudo code is matched by rule \#3 of the dictionary (figure 4.6). The $U A N$ pattern " $\$(\operatorname{varl} 1)+=1$ " cmbeds a variable denoted by " $\$(\operatorname{var} 1)$ ". Since the pattern may contain more than one variable, varl is a unique identifier name for that variable. The variable " $\$(\operatorname{varl})$ " is a plaitholder for the token which precedes the string " $+=1$ ". During the matching process, the variable " $\$(\operatorname{var} 1)$ " is 
bound to the token string which comes before the token sequence " $+=$ "”. In our example, " $\$(\operatorname{var} 1) "$ is evaluated to be "count" as a result of matching the UAN description "count $+=1 "$.

The corresponding Clock architecture pattern of rule \#3 is "inc\$(varl) | increment\$( varl)". This pattern gives Adligo a choice of either of two possible methods as the handler for "count $+=l$ ". The next section explains how these method names are derived from the corresponding L'AN description.

\subsubsection{The Pattern Language Usage}

As a simpler example, assume that the provided Clock architecture pattern is "inc\$(varl)", which is syntactically similar to the LAN pattern discussed above. Here, the variable "\$(varl)" is a reference to the variable used in the UAN partern " $\$(\operatorname{var} 1)+=1$ ". The Clock architecture pattern therefore evaluates to "incCount".

Next, - Halligo searches the current siope for this candidate method, inc Coint. Surprisingly, the result of this search is nil; i.e., there is no such method name in that scope. In rule \#3, the designer has in fact provided two alternatues: "inc\$(varl) | incrementS( varl)". The simbol "|" means OR, allowing the dictionary rule to provide multiple target handlers. Since the last search failed to find a method named inc Count, Adligo will automatically consider the next alternative, which is incrementCount.

The UAN match "count $+=1$ " has been written under the interface state column. Using a builtin rule, Adligo realizes that such a match should be handled by the bighest ADT in the current 
siope that has that member method. This rule leads Adligo to link the "count $+=1$ " match to the architecture handler Count.inirementCount (sec tigure 4.5 ).

\subsubsection{Linking Interface Feedback}

The third column in the LAN table is interface feedbac. This column contains the prose "Display Count". All the dictionary rules fail to find a match within this description. Nevertheless, the fact that this descripron falls in the user intertate feedhack column means the text specifies the system's output. Every component in the architecture has a men mechod that specifies its display. Since the current context component is still Cumbl iew, then any L'IN description in the interfact feedbatk column will be mapped to the niew method of the Coumt 'iew component. Note that the view method is not shown in the architecture herarchy (see figure $4.5)$.

Eventually, in the user actons of the second line, we encounter the action of releasing the mouse button " $\mathrm{M}$ "”. Generating the link for this action is the same as "MV", but this time rule \#2 is used for matching rather than rule \#1. At this point, the cntire table has been mapped. If there were more tables in the model, each would be processed.

\subsection{Link Generation Process: More Details}

This section highlights some concepts which Adligo users should be aware of. Section +.3 .1$ shows how it is important to identify the context in the UAN table, and section +.3.2 shows how a subtask referencing might change that context implicitly. Section 4.3 .3 explains the three logical classifications of the dictionary. Section 4.3.4 discusses the importance of the order in 
which the rules appear in the dictionary. Sections 4.3 .4 to +.3 .8 are concerned with the pattern language syntax and semantic.

\subsubsection{Changing the UAN Context}

The context change action, denoted by " [context]" is crucial to the link generation process because that acton means that context has ganed the tocus and has become responsible for handling the subsequent user acrions. If we can tind a component named context in the archirecture hierarchy, that component must be in charge of intercepting and handling all the subsequent user actions.

The LAN task model and the Clock architecture model are typically produced by different people. 'Therefore, an object named abi in the UAN description might exist in the (lock architecture but with a different name . $2 y$. Thus, Adligo needs a translation mechanism for determining that $a b c$ in the LAN notation means syq in the Clock architecture. The dictionary does this ransilation.

For example, figure +.7 shows the description of the task Reposition as Node. The HCI designer chose the name " $n$ " to express the node being moved, while in the Clock architecture, we find that the node is called nodel'iew. Rule \#6 in the CPMI Planner dictionary provides this translation (appendix B). 
As soon as Adligo recognizes the component that represents the UAN context, it will be able to derive the links for the UAN user actions. Adligo searches the architecture for the first handler for that user action in the current siope. Accordingly, Adligo realizes that " $M l v "$ and " $\mathrm{M}$ " " are handled by the handler browseNodemouseButton and " $\sim \mathrm{x}, \mathrm{y}]$ " by browseNoderel Motion. Notice that browse Node is not the context component, but lies in the siope of the current context component nodel "iesy.

This routing rule can be extended to the interfice state and connection to computation columns in a LAN table. These columns contain the $L A N$ descriptons most likely querying or changing the system's data. Such operations are handled by the architecture ADT"s, which are located in the siope of the context component. For example, in figure 4.8 , "lock $\mathrm{n}$ " in the intertate stute column is mapped to Lock. lock (by applying rule \#22 in appendix A - Standard Dictionary)

\begin{tabular}{|l|l|l|l|}
\hline & lock & lock \\
\hline
\end{tabular}

and "nodePos $(\mathrm{n}):="$ is mapped to NodePositions.setNodePosition (by applying rule \#) in appendix B).

The UAN description in the interface feedbatk column describes how the system's display changes as a response to the user actions. In chapter 3, we mentioned that Clock architecture is among those modem architecture styles that separate the input from the output, and that it has the purely specificational vien method that draws the component's display. This leads 


\begin{tabular}{|c|c|c|}
\hline USER ACTIONS & INTERFACE FEEDBACK & INTERFACE STATE \\
\hline $\begin{array}{l}\text { select mo } \\
\text { (move) }\end{array}$ & & \\
\hline$\left[-\left[\begin{array}{c}n \\
0\end{array}\right] \quad M V\right.$ & lock $\mathrm{n}$ & $\begin{array}{l}n ! \\
n \text { locked }\end{array}$ \\
\hline $1 \sim[x, y]$ & $\operatorname{nodePos}(\mathrm{n}):=(\mathrm{x}, \mathrm{y})$ & $\begin{array}{l}\text { display } n \text { at }(x, y) \text { on } \\
\text { all users' displays, } \\
\text { moving all cannected } \\
\text { lines and rubber-band } \\
\text { lines. }\end{array}$ \\
\hline $1 \star \mathrm{M}^{\wedge}$ & unlock n & $\begin{array}{l}\mathrm{n}-! \\
\mathrm{n} \text { unlocked }\end{array}$ \\
\hline
\end{tabular}

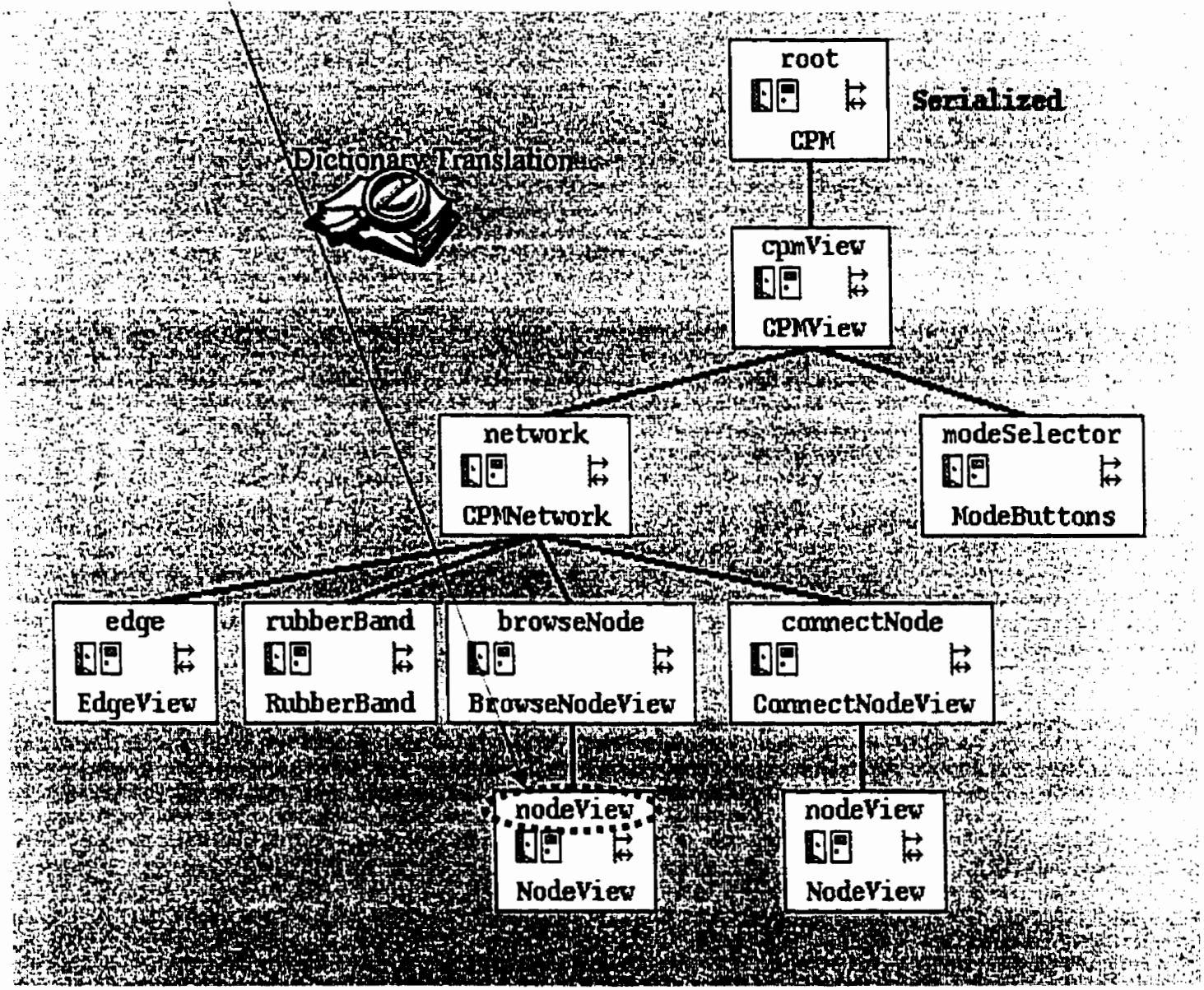

Figure 4.7. The figure shows the UAN table of the Reposition a Node task and the Clock architecture of the CPM Planner. The dictionary translates the UAN context " $n$ " to the "nodeView" component. 
Adligo to the fact that the UAN description in the interface feedback column should be mapped to the vies method of the context component. For example, "n!", "n locked", "n-!", "n unlocked" are all mapped to the handler nodel'iew.inew (see figure 4.8). Nevertheless, some exceptions for this rule may exist, as explained in section 4.3.6.

\subsubsection{Subtask Referencing in a UAN Table}

A subrask can be referenced in the user ations column. "Select Mode (Move)" in tigure 4.7 is an example of a subtask reference. Subtasks are treated as macros. The expanded task may contain user actions that change the context. Therefore, we have to make sure what is the current context at the end of that task. This is important because that context will become the context of the user actions immediately following that task reference. Fortunately, the "Select Mode (Move)" expansion in figure 4.7 is not critical in this LAN table because it is immediately followed by the acrion " $[\mathrm{n}]$ " which changes the context. Therefore, ignoring it will not cause any mistakes.

To guide Adligo to how a specific subtask affects the context, we write a rule in the dictionary that has the task name as the $U .4 N$ pattern, and the name of the context component as the Clock architecture pattern.

\subsubsection{Dictionary Types}

In practice, the dictionary is represented as a single text file. Logically, we classify the dictionary into three parts:

1. The Application-Specific dictionary consists of rules that are specific to an application under study. Appendices B and $C$ are examples of this dictionary type. 


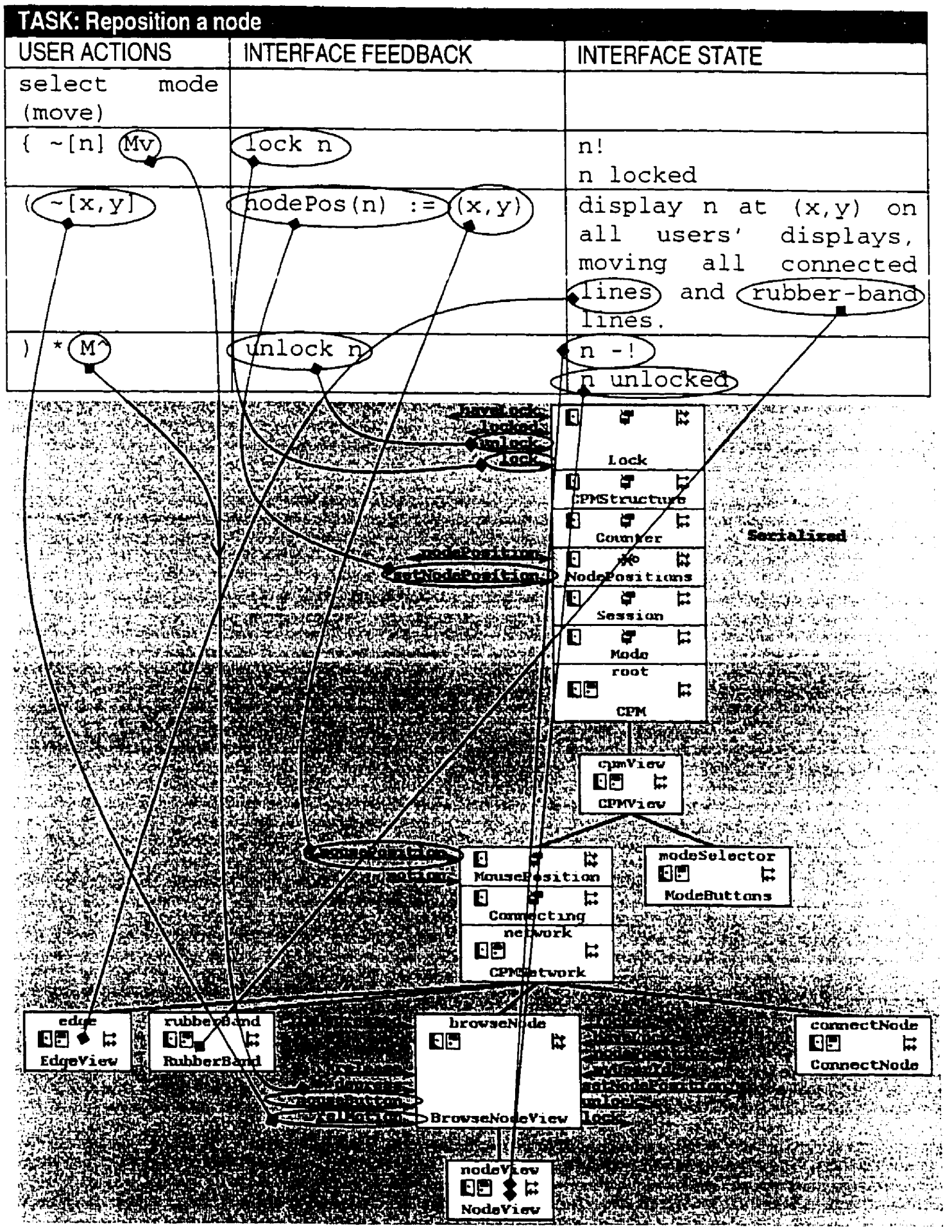

Figure 4.8. Some links that connect the "Reposition a Node" task to the CPM Planner architecture. 
2. The Component-Sperific dictionary consists of rules that are usually needed whenever a certain archirecture component is used.

3. The Stundard dictionary consists of rules that map the commonly used UAN notations to the predefined methods in Clock architecture. These rules are generic enough to be applied to any case study (see appendix $A$ ).

For simplicity, Adligo currently accepts one text file as a dictionary that contains the application-specific, the component-specific, and the standard dictionaries respectively: However, the implementation of Adligo could be enhanced by making Adligo include separate files of dicuonaries, in a similar way to including header files in the $\mathrm{C}++$ language or imporring packages in Java.

\subsubsection{Searching the Dictionary}

The dictionary is sequentially searched from top to bottom. The first matchable rule is selected and used to derive a link. This allows some rules to overwrite others already there.

For example, "currentMode := mode" in figure 4.9 shows that the match "currentilode :=" is linked to Mode.setMode. Rule \#10 in appendix B helped to generate this link correctly.

currentMode := setMode

If this application-specific rule were not added, this matching could be possible by rule 28 


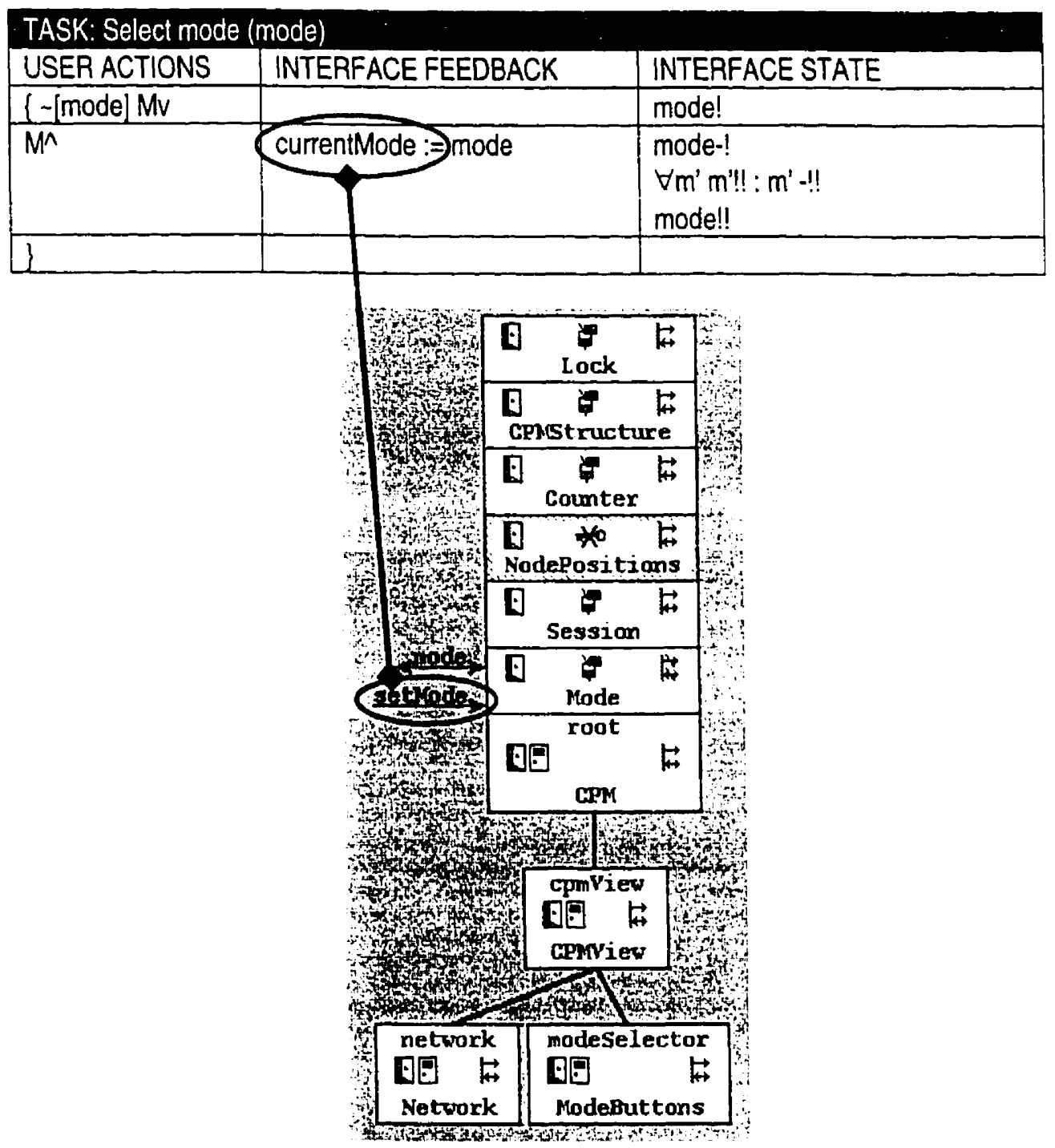

Figure 4.9. "currentMode $:="$ is linked to Mode.setMode.

in the standard dictionary (see appendix $A$ ), but that match would lead to the composition of a non-existing method name "setCurrentMode", which means no link would be generated. Therefore, the order in which the rule appears in the dictionary is sometimes significant. 


\subsubsection{Using Variables in Patterns}

The simplest pattem in this language is a string literal, which is used in rules translating a LAN context name to a Clock component name (see rules \#3 and \#+ in appendix B).

\begin{tabular}{|l|l|l|}
\hline & $\mathrm{n} 1$ & nodeView $\rightarrow$ connectNode \\
\hline & n2 & nodeView $\rightarrow$ connectNode \\
\hline
\end{tabular}

A pattern may contain one or more variables. The variable takes the form of $\$($ variableName). The variablename is a unique identifer, used for later reference. The meaning of the variable is dependent on whether it is part of a L'AN pattern or a Clock architecture pattern. If the variable is part of the U.N partern, then it plays the role of a plactholder. The value of the variable is bound during a successful match with a LAN description. The value of this variable is the string that composes or completes a token in the pattern. If the variable is part of the Clock architecture pattern, then it should have been used before in the LAN pattern. The value of this variable is substinuted inside the Clock architecture pattern to form a string literal (see section +.2 .8 for a complete example).

Rule \#12 in appendix $B$ shows that it is not necessary to reference the U:AN pattern variables in the Clock architecture pattern.

Such variables are used as dummy placeholders, but they are still important to the formation of such a generic rule. 


\subsubsection{Handler Names}

We can write a rule which explicitly specifies a link, i.e., a rule which maps a U.AN pattern to a particular Clock architecture handler. Syntactically, using the operator ".", we can write the handler name as componentName. method or ADTName.method in the Clock architecture pattern.

For example, in the Reposition a Node task (figure 4.8), we show that some feedback matches such as "n!", "n unlocked", and "n-!" are linked to the handler nodel "iew. mew, where nodel "i'w represents the context component. This intuitive mapping does not apply to "lines" and "rubber-band lines" in the second line of the table. That is because the correct mapping of "lines" is to edgeniew and "rubber-band lines" to mbberbundmen (figure +.8). We use the dictionary rules \#13 and \#1+ to explicitly map such matches correctly.

\begin{tabular}{|l|l|l|}
\hline & lines & edge.View \\
\hline & rubber-band & rubberBand.view \\
\hline
\end{tabular}

\subsubsection{Chained Component Names}

Tivo components in the Clock architecture are allowed to have the same name if they are in different scopes. This might cause a problem for Adligo in determining which component to choose during the search process. Such ambiguity was also present in the Repostion a Node task as we translate the UAN context " $n$ " to nodel iew. But as shown in figure +.7 , the Clock architecture has two components named node ['iew.

To resolve this ambiguity, we specify as many parents of the intended component as necessary to remove the ambiguity. The pattern language has the operator " $\rightarrow$ " which specifies the 
component's parent. The component name may appear in the pattern as a chain, which looks like

$$
\text { comp } \rightarrow \text { parent: } \rightarrow \text { parent }_{2} \rightarrow \ldots \rightarrow \text { parent. }
$$

Rules \#6 and \#3 in appendix $B$ are examples of rhis tyras.

\begin{tabular}{|l|l|l|}
\hline Reposition a node & $n$ & nodeView->browseNode \\
\hline & n1 & nodeView->connectNode \\
\hline
\end{tabular}

\subsubsection{Alternatives in the Clock Architecture Pattern}

The pattern language has the operator "|" that means ()R, allowing the dictionary rule to provide multiple target handlers as alternatives in the Clock architecture pattern (see Rule \#6 in appendix $A$ ).

\begin{tabular}{|l|l|l|}
\hline & $\sim$ \$(obj1), \$(obj2)] & motion I relMotion \\
\hline
\end{tabular}

Adligo considers one alternative after another during its search. Upon finding the first instance of any alternative, Adligo creates a link. For example, linking the $U A N$ match " $-[x, y]$ " was possible by using rule \#6 whose Clock architecture pattern is "motion | relMotion". This match was linked to browseNode.re/Motion because it is lower than Mousel Position.motion in the current scope (see figure 4.8 ). 


\subsubsection{About the Design of the Pattern Language}

Simplicity and ease of use were the top concerns in the design of the partern language (see the its grammar in appendix D). This language has just one type of variables, and three operators (")", " $\rightarrow$ ", and "."). For the sake of ease of use, we avoided to use two different notations of using variables (see section t.3.5) in the L'AN and the Clock architecture patterns. In addition. this notation "\$(var)" is inspired by the notation used to write variables in the Mlake files of the LNIX operating system.

As discussed in chapter 6, Experiments and Analysis, we found this pattern language adequately flexible to write dictionary rules that generate high accurate links. We could obtain a large percentage of links $\left.(9)^{(0)}\right)$ by writing small number $(8)$ of simple rules. The pattern language was efficient enough to write advanced rules to get $99^{\circ}$ o of the available links.

\subsection{Summary}

This chapter provides Adligo users with a full description of the link generation process. It starts by giving a general description of the framework of this process. It then shows where the Adligo tool fits into this frame, and what inputs should be submitted to it.

The second part of the chapter helps the user to understand how to generate the links systematically by following a simple tutorial. The tutorial provides a complete explanation for deriving all the links of a simple case study. This tutorial also introduces some aspects related to the link generation process such as the importance of the U.N context in link inference, 
the scoping rules in the Clock architecture, Adligo's built-in knowledge, and the pattern language.

The third part of the chapter proceeds in providing more details about the role of the L'AN context in deriving most of the links. It shows how some subtask references can alter the L:WN context. This part presents the three logical classifications of the dictionary: applicataonspecific, component-based, and standard. It also shows how the order of the rules inside the dictionary can affect the correctness of the generated links. The syntactic and semantic details of the pattern langunge are discussed such as using variables embedded in the pattern, specifying handler names, and the need for the chained component names. Finally, a simple analysis is given about the design for the pattern langunge and its usage. 


$$
\text { Chapter } 5
$$

\section{LOOKING UNDER THE HOOD:}

\section{THE IMPLEMENTATION OF ADLIGO}

Chapter + gave Adligo users a detailed description of the link generation process. It presented a simple tutorial for deriving links between the $\mathrm{U}$ IN and the Clock architecture models. Since the presentation in that chapter targeted Adligo users, no implementation details were discussed. This chapter presents implementation details of Adligo.

Section 5.1 provides a technical description of the framework of the link generation process and defines the basic data types and mathematical notations used in the rest of the chapter. Section 5.2 describes the dictionary functiorss. Section 5.3 describes the message routing rules of the Clock architecture style. In section 5.4, we describe how Adligo derives links for the UAN aser attions column. In section 5.5, we describe how Adligo derives links for the L'AN interfice state and connection to computation columns, while in section 5.6 we describe the link generation algorithms for the UAN interface feedback column. 


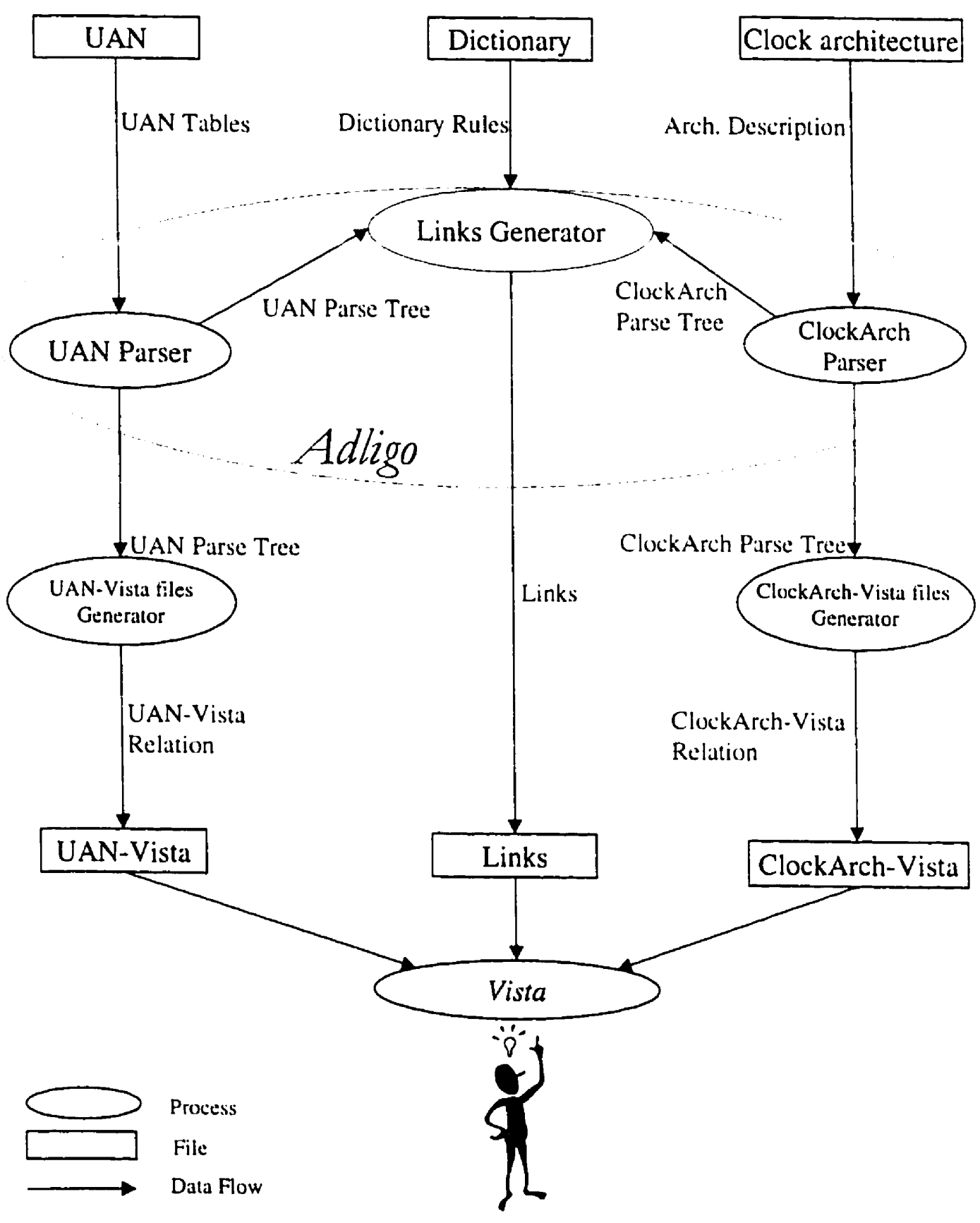

Figure 5.1. The bighb-level design of the link generation processs using the Adligo tool. 


\subsection{The Framework of the Link Generation Process}

As shown in figure 5.1, the link generation process requires three inputs: the LAN model, the Clock architecture model, and the dictionary. The output is a set of links that map elements of each model to the other.

At present, we write the UAN model manually in a text file because we do not have a L'AN editor. Appendix E describes the grammar we use to write the UAN notarion in that file. Since we use the visual programming environment ClocklE"ork; [GR.tHtM et al., 1996a] to write our Clock programs, the Clock architecture is easily obtained from ClockWorks as a text file. Fach line written in this tile describes a component in the architecture or the relationship between components in the hierarchy. Appendix F specifies the grammar used to parse this tile.

Both the $[A N$ and the Clock architecture models are fed into the Idligo tool. Adligo has a parser for each model that produces the parse tree. The two parse trees are input to the Adligo's internal module, the link gentrator, to establish the links between the two trees.

The link generator traverses the L.AN parse tree, trying to generate a link for every L IN matith. A U.AN march is any sequence of tokens in the UAN description that can be matched by a dictionary rule, and therefore mapped to the Clock architecture. The direction of processing inside a $U A N$ table is the same as the way it is read: top to bottom, left to right.

The UAN columns are processed in the following order: user actions, interface state, intertace feedback and finally connettion to computution. In order to find a match, all the UAN symbols inside 
the table are parsed as tokens. A token can be a number (a sequence of digits), a string literal (a sequence of letters), or any other symbol. We define a token using the regular expression:

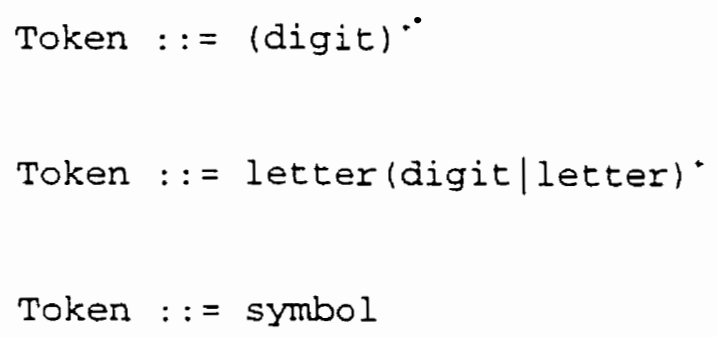

where digit is any one of $0,1,2,3,4,5,6,7,8$ or 9 , letter is any alphabetic character (any one of a-z or $\mathrm{A}-\mathrm{Z}$ ) or _ (underscore), and symbol is any keyboard character which is not a digic nor letter.

To describe how Adligo identifies a U:N match and maps it to a Clock architecture handler. we use the notation $<t>$ to define a sequeme of type $t$. If $t s$ is a sequence of tokens inside one of the UAN columns, the link generator searches the diccionary rules sequentially, top to bottom, to find a rule whose LAN pattern tokens (ts') can match any subsequence in es. (Notice that the LAN pattern of every rule is also parsed into a sequence of tokens ts'.) As soon as that match is found, the matched tokens in ts become a UiN match, and the link generator gets its translation from the Clock architecture pattern column. This L'AN match may be linked to an architecture handler. Searching for more UAN matches among the unmatched UAN tokens of $t s$ continues undil all the dictionary rules fail to get a match.

Figure 5.2 provides a simplified description of the process of gencrating a link between the UAN model and the Clock architecture. Adligo translates a UAN context to a context component by looking up the dictionary. A UAN match is translated to a set of candidate 


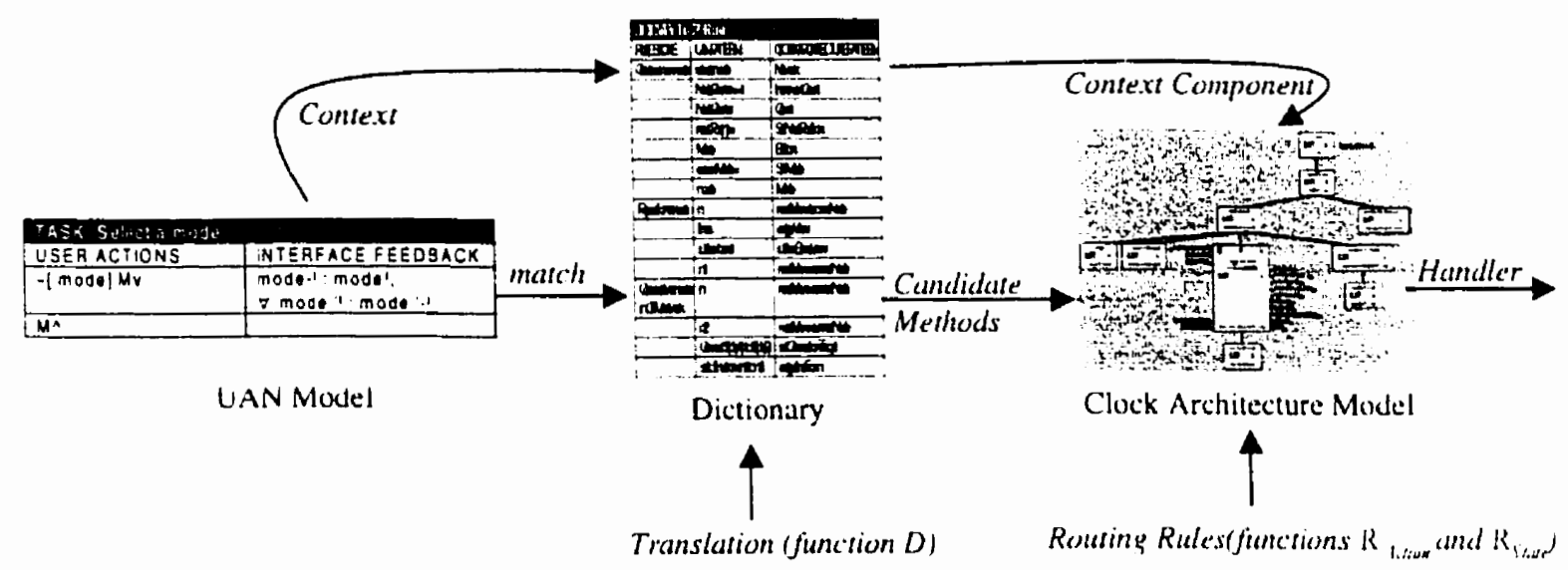

Figure 5.2. Generating a link between a UAN match and a Clock architecture handler. Using the dictionary, Adligo translates a UAN context to a context component, and a UAN match to a set of candidate methods. To find the correct handler. Adligo uses the routing rules of the Clock architecture style.

methods through the dictionary function $D$. The roucing rules of the Clock architecture strile use this set of candidate methods and the context component to find the correct handler in the Clock architecturc.

In subsequent sections, we describe how Adligo generates links for the different L'AN columns. Whenever appropriate, we support our discussion by mathemarical notarion to describe the algorithm accurately. Thus, we need to define common types which are used in later discussion.

Context represents the set of UAN contexts; Component represents the set of components in the Clock architecture; ADT represents the set of ADT's in that architecture, and Method represents the set of update and request events which a component or an ADT may receive. $A$ link maps a UAN match to a Clock architecture handler. We define a match as a sequence of tokens: 


$$
\text { Match }==\langle\text { Token }>
$$

and a handler as a method inside a component or ADT:

$$
\text { Handler }==(\text { ComponentorADT } \times \text { Method })
$$

where ComponentOrADT $==$ Component $\cup$ ADT .

(Figure 5.3 illustrates the meaning of some terms.) Accordingly, we define links as a relation between matches and handters:

$$
\text { Link }==(\text { Match } \times \text { Handier })
$$

\subsection{The Dictionary Functions}

In this section, we define the dictionary functions, which we use in subsequent sections to

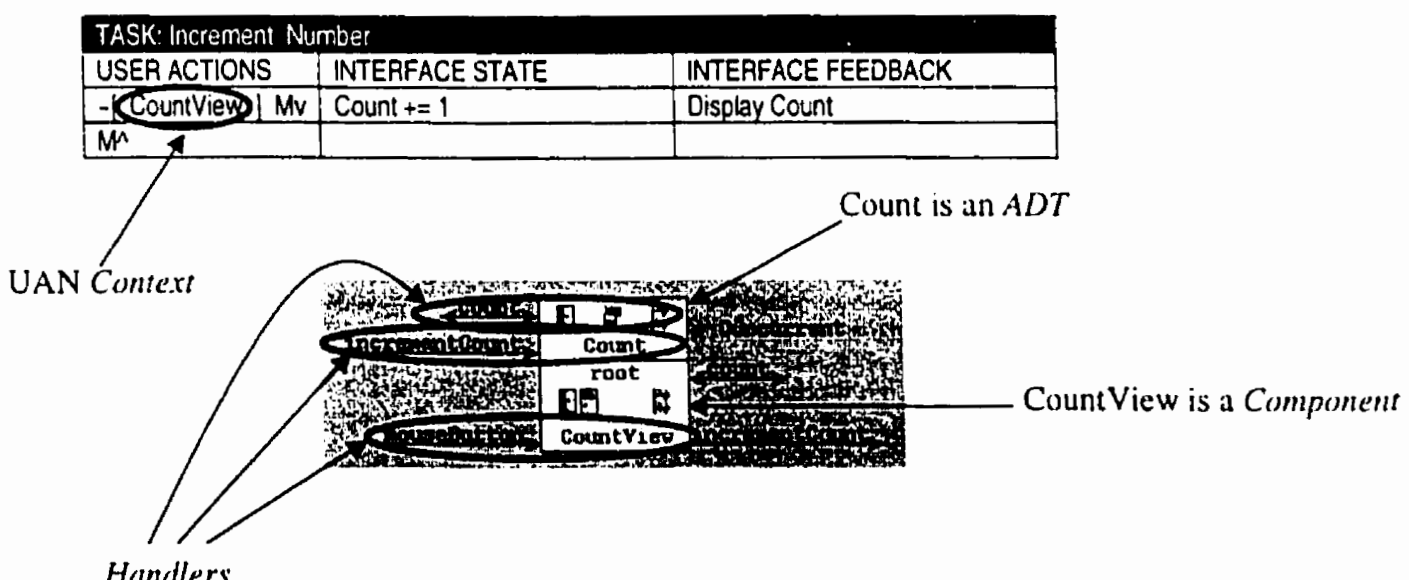

Figure 5.3. In UAN, a Context appears in the notation $\sim$ [Context]. In this Clock architecture, the root component is augmented by the Count ADT. A Clock architecture Handler is a method inside a Component or an ADT. 
define Adligo's link generation functions. The dictionary has two funcrions:

(i) Funcrion $C$ maps a UAN context to a Clock architecture component.

(ii) Function D maps a LAN match to a set of components, ADT"s, methods, or handler names which might exist as implementations of that L'AN match in the Clock architecture.

D simply implements a table lookup in the dictonary: given a L'AN match, the corresponding Clock architecture partern is returned. Since these semantics are straightforward, we do not define $D$ formally. $C$ can be defined in terms of $D$. Tu describe $D$, we assume that the simbol $\perp$ ('bottom') is a distinguished element used to represent a null value. (i.e., if $S$ is a set, then $S_{\perp}$ $=S \cup\{\perp\})$.$P denores the power set. The dictionary sequentially searches (top to bottom) for$ a matching rule using the $\mathrm{D}$ function, which has the following signature:

\section{D: Match $\rightarrow$ P(ComponentOrADT $\perp \times$ Method $\left._{1}\right)$}

D takes a LAN match as argument and maps it to a set of components, ADT"s, methods, or handlers. The function returns a set because the pattern language allows a UAN match to be mapped to multiple potential targets in the Clock architecture (see section 4.3.8).

$C$ has the following signature:

$$
\text { c: Context } \rightarrow \text { ContextComponent }
$$


Chapter 5: Looking Under the Hood: The Implementation of Adligo

where Context $\subseteq$ Match and ContextComponent $\subseteq$ Component.

ContextComponent represents the Clock architecture components which may be a translation of the $\mathrm{UAN}$ context.

The $C$ function accepts a $L A N$ match (ts) representing the LAN context, or a referenced subtask which causes changing the context (see section 4.3.2), and translates ts to an architecture component $e$. If $t s$ is not found in the dictionary, the function returns $\perp$. $C$ is detined as:

$$
C(t s)=\left\{\begin{array}{l}
e, \text { if } \exists e \in \text { ContextComponent } \bullet D(t s)=\{(e, \perp)\} \\
\perp, \text { otherwise }
\end{array}\right.
$$

\subsection{The Routing Functions}

The routing functions in the Clock architecture play an important role in detining the link generation functions which Adligo implements. Since these functions were defined formally in [GRLHAM, 1995], we do not define them here, but we define Adligo's functions in terms of them. There are rwo routing functions in the Clock architecture style:

(i) Function $R_{\text {tenun }}$ searches the scope of the context component to find the first component or ADT that implements the first instance of any method among the multiple targets mentioned in the Clock architecture pattern. We use this routing function to define the functions which generate links for the user attions column. 
(ii) Funcrion $\mathrm{R}_{\text {tarte }}$ which searches for the first ADT in the context component scope that implements one of the methods mentioned in the Clock architecture pattern. W'e use this routing function to define the functions which generate links for the interface itute and connetion to computation columns.

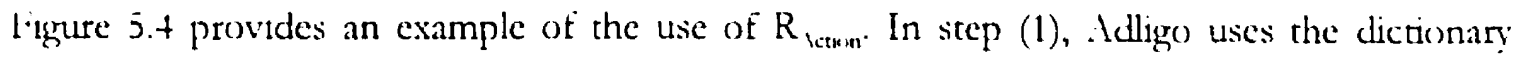
function $C$ to translate the $\mathrm{U}: \mathrm{N}$ context (" $n$ ") to the Clock architecture component ("nodeView") using the following rule:

\begin{tabular}{|l|l|l|}
\hline & $n$ & nodeView \\
\hline
\end{tabular}

In step (2), Adligo uses the D function of the dictionary to translate the LAN match " $-\mid x, y$ " to al set of candidate methods \{relMotion, motion\} using this rule:

\section{$\sim[\$(o b j 1), \$(o b j 2)][$ relMotion I motion}

Adligo uses the $\mathrm{R}_{\text {detum }}$ function to tind the first component or ADT which has the tirst instance of these methods. Since the "MousePosition" ADT has the method "motion", this makes ("MousePosition", "motion") the correct handler. $\mathrm{R}_{\text {Ictu }}$ hats the following signature:

$$
R_{\text {Action }}: P(\text { Method) } \times \text { ContextCompnent } \rightarrow \text { Handler. }
$$




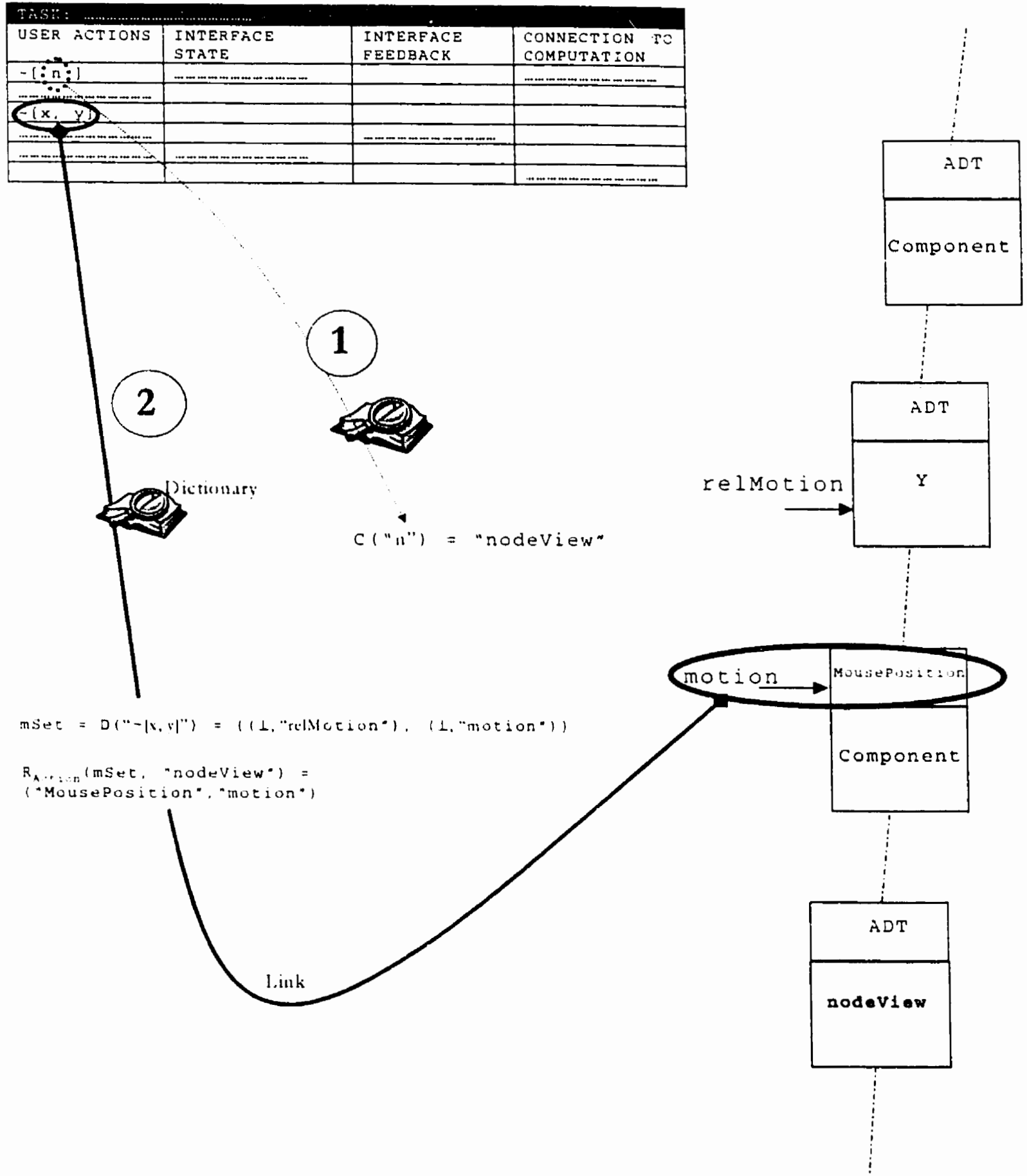

Figure 5.4. Generating links for user actions. Step (1) translates the UAN context to a component. Step (2), Adligo uses the dictionary function $D$ to translate the match " $-[x, y]$ " to a set of multiple target methods represented by the set $m S e t . \mathrm{R}_{\text {lition }}$ seeks the first component or $A D T$ which intercepts the first instance of any method in mSet. 
The first argument is a set of candidate methods resulting from translating a UAN match using

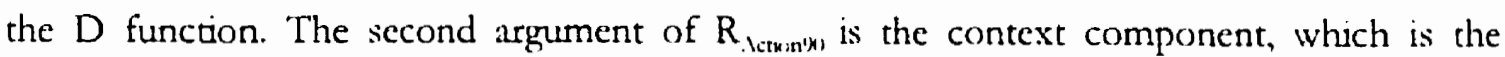
translation of the UAN context.

Figure 5.5 provides an example of the use of $R_{\text {tiate }}$ In step (1), Adligo uses the dictionary funcrion C to translate the L'AN context " $n$ " to "nodeView" using the rule:

\begin{tabular}{|l|l|l|}
\hline & $n$ & nodeView \\
\hline
\end{tabular}

In step (2), Adligo uses the D function of the dictonary to translate the L'AN match "count $+=1$ " to a set of candidate methods \{incCount, incrementCount $\}$ using this rule:

\begin{tabular}{|l|l|}
$\$(\operatorname{var} 1)+=1$ & inc $\$(\operatorname{var} 1) \mid$ increment $\$(\operatorname{var} 1)$ \\
\hline
\end{tabular}

Adligo uses the $\mathrm{R}_{\text {stat }}$ funciton to find the first $\mathrm{ADT}$ which implements any method in miset. The "Counter" ADT implements the method "incrementCount". This makes ("Count.increment", "Count") the correct handler. $R_{\text {satt }}$ has the following signature:

$$
R_{\text {state }}: P(\text { Method) } \times \text { ContextComponent } \rightarrow \text { ADT } \times \text { Method. }
$$

$R_{\text {state }}$ searches the Clock architecture for the correct handler (ADT, method). The tirst argument is a set of candidate methods resulting from translating a CAN match using the D function. The second argument of $R_{\text {stare }}$ is the context component, which is the translation of the UAN context. 


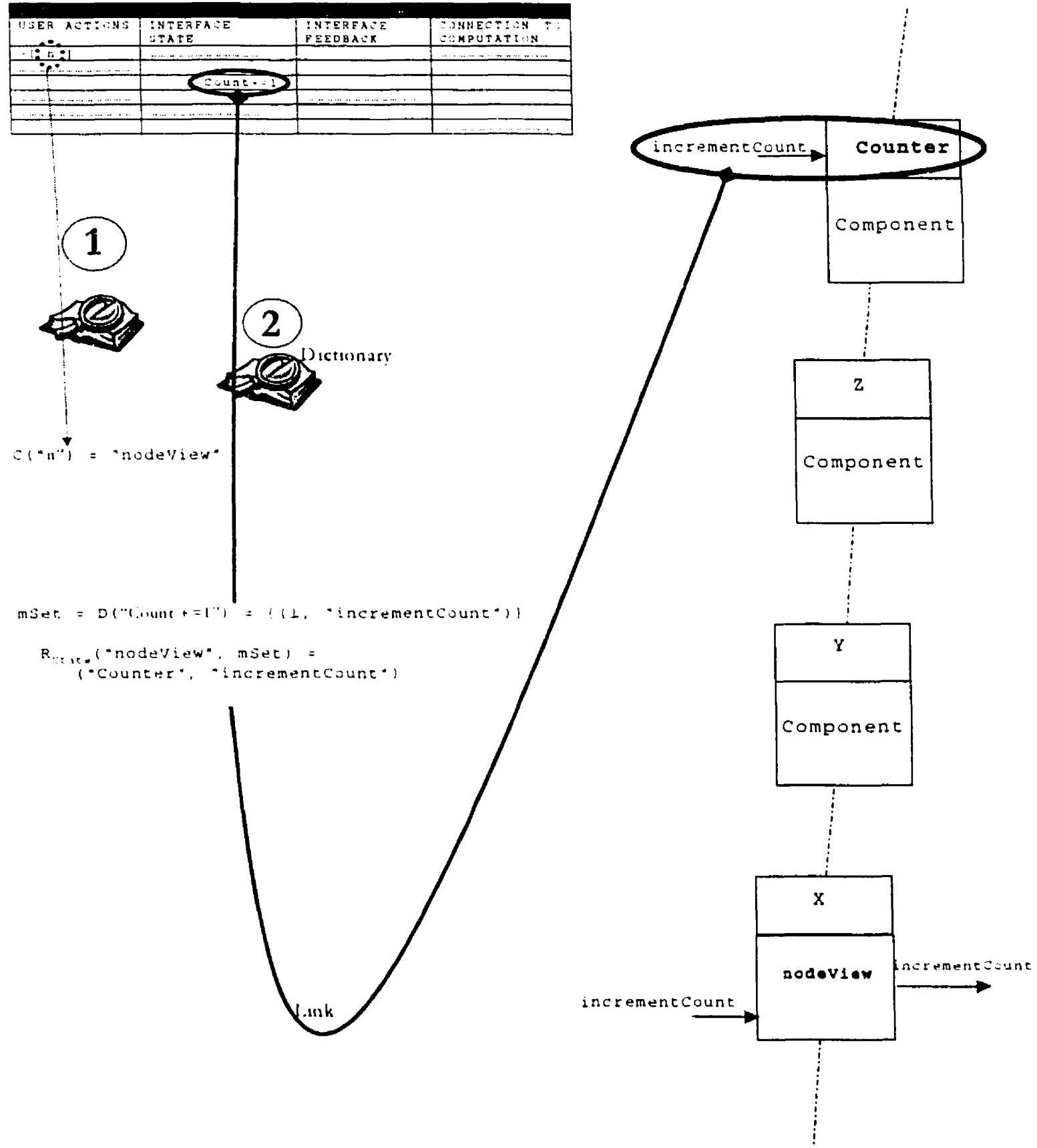

Figure 5.5. Generating links for interface state or connection-to-computation columns. Step (1) translates the UAN context to a component. In step (2), Adligo uses the dictionary fiunction $D$ to translate the "count $+I$ " match to a set of candidate methods mSet. Adligo uses the $R_{\text {siute }}$ routing function to find the first ADT that implements any method in $m$ Set, starting from the current context of "nodeView". 
In the following sections, we define Adligo's link generation functions in rerms of these routing functions.

\subsection{Linking User Actions}

Identifying the context component in the Clock architecrure is significant to the link generation process. Therefore, Adligo maintains an internal system variable contextComponent which represents the translation of the current LAN context. In the user actions column, the link generator keeps track of two important user actions: the context change action denoted by " [newContext]" and subtask referencing (see secrions +.3.2). Both can alter the LAN context, causing Adligo to translate the LAN context to an architecture component (using the dictionary function C) and to assign this translation to the varable contextComponent.

For other user actions (which have no impact on the U.AN context), the link generator looks up the dictionary for a translation using the $D$ function, and uses the routung function $R_{\text {tetewn }}$ to find the correct handler for this user action.

We can describe the signature of the function $G_{1}$ which generates the links for a sequence of user actions in a given component context as follows:

$G_{A}:<$ Match $>\times$ ContextComponent $\rightarrow P$ Link 
$G_{1}$ takes two arguments. The first is a sequence of user action matches, and the second is the context component. The function returns a set of links associating that sequence of user actions with the Clock architecture.

To define this function, we identify those sequences of tokens which represent a subtask name; i.e., we let Subtask $\subseteq<$ Token>, and we assume: $c \in$ ContextComponent, $c$ ' $\epsilon$ Context, $a \in$ Match, as $\epsilon<$ Match>. Then we define the function $G$, through the following set of equations:

$$
G_{\dot{A}}(<>, c)=\varnothing \text {, }
$$

if no user actions are present, no links are generated.

$$
G_{A}\left(<\sim\left[c^{\prime}\right]|a s\rangle, c\right)=G_{A}\left(a s, c\left(c^{\prime}\right)\right)
$$

$\sim\left[c^{\prime}\right]$ is the change context action. This action is not linked but the remaining actions $(a s)$ should be linked with respect to the new context component $C\left(c^{\prime}\right)$. The notation $\langle\mathrm{X} \mid \mathrm{XS}\rangle$ represents a scquence in which $\mathrm{X}$ is the first element and $\mathrm{Xis}$ represents the sequence of the remaining elements.

$$
G_{n}(\langle a \mid a s\rangle, c)=\left\{\begin{array}{l}
G_{A}(a s, C(a)), \text { if } a \in \text { Subtask } \wedge C(a) \neq \perp \\
G_{A}(a s, C), \text { if }(a \in \text { Subtask } \wedge C(a)=\perp) \vee D(a)=\varnothing \\
\left\{\left(a, R_{A c t i o n}(D(a), C)\right)\right\} \cup G_{A}(a s, c), \text { otherwise }
\end{array}\right.
$$


Choice no. 1: If $a$ is a subtask reference that has a translation in the dictionary, then the remaining user actions as are linked with respect to the new context component $C(a)$. Choice no. 2: If a is a subtask reference that has no translation, or a is another user action with no mapping rule in the dictionary, then $a$ is ignored and the remaining user actions as are linked with respect to the current contexr. Choice no. 3 : If the previous conditions are not satustied, then the result of the function $G_{1}$ is the set of links resulting from mapping the acrions a and as.

\subsection{Linking Interface State and Connection to Computation}

The UAN description in the interface stute and commetion to computation columns mainly describes the system's data. Therefore, most of the U.AN matches in these columns are mapped (o) methods inside ADT's. The same link generation rules apply to these columns and we describe the link generation rules in terms of the interfice state column.

We describe the $G_{\mathrm{s}}$ function which generates links for the intertace state column by the following signature:

$$
\mathrm{G}_{\mathrm{S}}:<\text { Match }>\times \text { ContextComponent } \rightarrow \mathrm{P} \text { Link }
$$

$\mathrm{G}_{\mathrm{s}}$ takes two arguments. The first is a sequence of interface state matches, and the second is the context component. The function returns a set of links associating that sequence of interface state matches with the Clock architecture. To define this function, let us assumc $c$

$\epsilon$ ContextComponent, $s \in$ Match, $s s \in<$ Match $>$. $G_{s}$ is then defined through the following equations: 


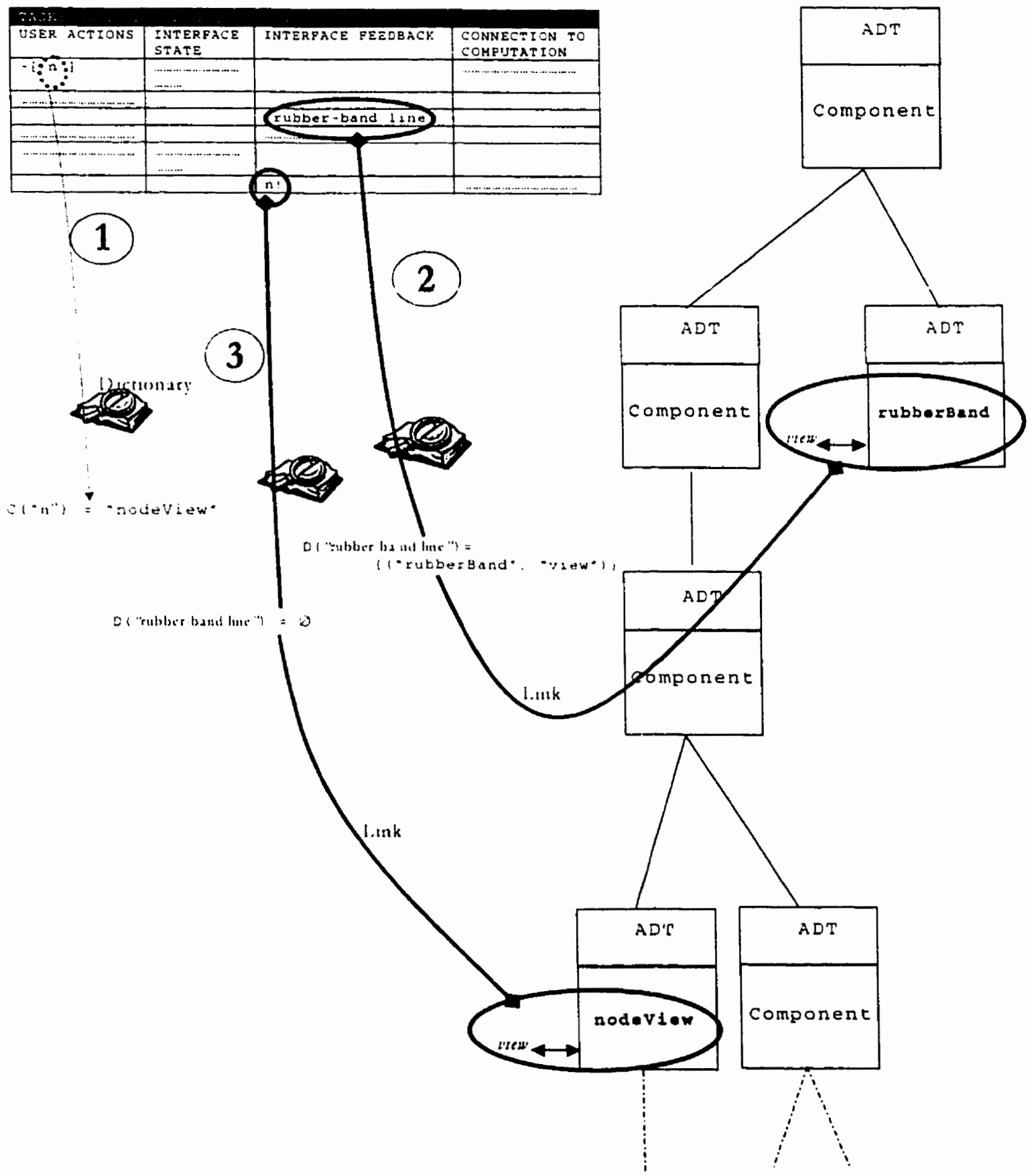

Figure 5.6. Generating links for the interface feedback column. In step(l), Adligo translates the UAN context " $n$ " to the component "nodeView". In step(2), Adligo translates the "rubber-band line" using the dictionry fucntion D to the explicit handler ("rubberBand", "view"). In step (3), Adligo finds no translation for the " $n$ !" match, so it links it, by default, to the "view" method of the context component "nodeView". 


$$
G_{S}(<>, c)=\varnothing,
$$

if no interface state matches are present, no links are generated,

$$
G_{s}(\langle s \mid s s\rangle, c)=\left\{\begin{array}{l}
G s(s s, c), \text { if } D(s)=\varnothing \\
\{(s, \operatorname{Rstace}(D(s), c))\} \cup G_{s}(s s, c) \text {, otherwise }
\end{array}\right.
$$

Choice no.1: If there is no dictionary rule which matches the interface state $s$, then ignore it, and generate links for the remaining interface state matches ss. Othervise, in choice no. 2, the result of the function is the set of links generated for the interface state $s$ and for $s s$.

\subsection{Linking Interface Feedback}

Lastly, in the interficie feedlucik column the link generator in Adligo searches the dictionary for a march. If found, the dictionary translation should be a handler: the component name and its wiew method (figure 5.6, step(2)). However, the Adligo user does not need to explicitly map every feedback description, because Adligo, by default, links it to the wien method of the context component (figure 5.6, step(3)). We describe the function $G_{1}$ which generates the links for the interface state matches by the following signature:

$$
\mathrm{G}_{\mathrm{F}}:<\text { Match }>\times \text { ContextComponent } \rightarrow \mathrm{P} \text { Link. }
$$

'To define this function, we assume $i \in$ ContextComponent, $\imath^{\prime} \in$ Component, $m \in$ Method, $f$ $\in$ Match, fs $\in$ <Match>. We define $\mathrm{G}_{\mathrm{l}}$ through the following equations:

$$
G_{F}(<>, c)=\varnothing
$$


if no interface feedback matches are present, no links are generated,

$$
\begin{aligned}
& G_{F}\left(<f \mid f_{S}, c\right)= \\
& \qquad\left\{\begin{array}{l}
\left\{\left(f,\left(c, " v i e w^{\prime \prime}\right)\right)\right\} \cup G_{F}(f s), \text { if } D(f)=\varnothing \\
\left\{\left(f,\left\{\left(c^{\prime}, m\right)\right\}\right)\right\} \cup G_{F}(f s), \text { where }\left\{\left(c^{\prime}, m\right)\right\}=D(f) \text {, otherwise }
\end{array}\right.
\end{aligned}
$$

Choice no. 1: is the default set of links resulting from mapping the $f$ match to the context component $(c)$ plus the links that map the remaining interface feedback matches fs. Otherwise, in choice no. 2, if $f$ is matched by a dietionary rule, then the function returns a set of links consisting of a link that maps $f$ and the links mapping the remaining interface feedback marches $f_{s}$.

The process continues line by line until it finishes an entire LAN table. Adligo proceeds to the next table until all the UAN model tables are mapped. At that moment, the link generation process terminates. The final result is a set of links associating the L':LN model to the (lock architecture. Adligo writes these links to a text file.

In order to display visually the results, we use the l'istu tool [BROW'N et al, 1998]. V'ista needs specially formatted files in order to be able to demonstrate the links between the LAN model and the Clock architecture. It needs some files that represent the CiAN and the Clock architecture models. In order to generate these files, the parse trees of both models are passed to the Clock-1nth.l'ista files generator and the UAN-Lista files generator. The generated links are also formatted properly to be read by Vista. Lipon preparing this set of files for Vista, we can graphically navigate the links associating the two models. 


\subsection{Summary}

The chapter was concerned with implementation aspects of the link generation process. It technically explained the general framework of this process. It described one primitive function (D) for the dictionary, and two of the routing rules $\left(R_{\text {lcanm }}\right.$ and $\left.R_{\text {tate. }}\right)$ existing in the Clock architecture style. $D$ is a simple table look-up. The routing funcrions were formally specified [GRtHAI, 1995]. We used these predefined funcrions to define the funcrions which Adligo implements to derive links for the various LAN table columns. That description was intermingled with some mathematical notations in order to express the concepts formally.

From the technical discussion presented in this chapter, we norice that Adligo has litele knowledge about the $\mathrm{LAN}$ and the Clock architecture models. This intemal knowledge is hard-wired inside Adligo as built-in rules. The first rule relies on the notion of identifying the UN context and gets its translation in the Clock architecture. The second rule relies on using the routing mechanisms of the Clock architecture style. Any other rules needed to generate links can be externally written in the dictionary by Adligo users. 


$$
\text { Chapter } 6
$$

\section{EXPERIMENTS AND ANALYSIS}

- Lelligo is the computer-based tool that has been developed to support this thesis's hypothesis. It accepts a LAN task model and a Clock architecture, and links them together with the assistance of a third input, which is the ditionary.

This chapter reports on two case studies involving these applications: a project planner and a rideo annotator. While these case studies show that Adligo is able to derive links with high accuracy and completeness, further experiments would be required in order to conclude that similarly positive results would be obtained for other applications.

This chapter is organized as follows. Section 6.1 discusses the measures which we used to evaluate - Idligo's performance. Section 6.2 describes the experiments and the case studics which we performed. In section 6.3, we review our experimental method. Section 6.t gives the raw results, and section 6.5 concludes this chapter with an analysis. 


\subsection{Measures}

The main goal of our experiments is to get a sense of the difficulty of using Adligo as an automated tool and the accuracy of the links generated by Adligo. Towards this goal, we detine the following. Assuming Adligo is run on some task and architecture models,

$\mathrm{I}_{\tau}$ : represents the ser of links generated by the . Idligo tool.

$\mathrm{I}_{11}$ : represents the definitive set of links against which we compare the links genernred by Acllige. This set of links is created by a human (manually). Secrion 6.3 explains how we prepared $L_{\imath 1}$ for our two major case studies.

We will compare $L_{4}$ against $L_{11}$. We measure the utility of Adlige based on nvo criteria: case of use and accuracy.

\section{1) EASE OF LSE}

This is measured in terms of the number of rules that designers need to add to the appiliationspecific dictionary. The stundard and componemt-besed dictionaries are excluded because they are automatically included in every application and therefore cause no burden to Adligo users. We observe how many customized rules we need to write to get a high rate of correct links $\left.(9)()^{\prime \prime}\right)$ and to get aimost all the correct links $(99 \%)$.

\section{2) ACCLRACY}

The accuracy of Adligo's results can be measured in terms of two rates ("ll " denotes set cardinality): 
1) Coverage Rate:

$$
\text { Coverage Rate }=\left\|\mathrm{L}_{\mathrm{c} i} \cap \mathrm{L}_{11}\right\| /\left\|\mathrm{L}_{1 \mathrm{i}}\right\| \times 100 \%
$$

This gives the percentage of the correct links generated by -1diligo.

2) Error Rate:

$$
\text { Error Rate }=i i L_{c_{i}}-I_{-11} i j / i i L_{4 i} i \mid \times 1000^{\omega}{ }_{0}
$$

This represents the percentage of links generated by -1 dllige which are incorrect. $A$ link is considered incorrect if it is not present in the set of links found by the human. If designers are using $l$ ista to browse the links that. Ldligo has generated, this rate tells them the likedihood of a chosen link mapping them to an incorrect part of the opposite model.

\subsection{Experiments}

- Ldligo has been tested using four small to medium sized case studies. The small case studies are the Counter and the l'ideo Pluger applications. The medium case studies are the (iritial Pat) Method (CPM) Planner [GRUHAM, 1997] and the lideo . Imnotutor [GR.AHAM AND LRVES, 1997]. Table $6 .+$ gives an idea about the size of these case studies in terms of the number of tables and lines in the UAN model, and the number of components, ADT's and methods in the Clock architecture.

\begin{tabular}{|l|l|l|l|l|l|}
\hline & UAN Tables & UAN Lines & Components & ADT's & Methods \\
\hline CPM Planner & 18 & 122 & 13 & 11 & 92 \\
\hline Video Annotator & 19 & 122 & 30 & 20 & 133 \\
\hline
\end{tabular}

Table 6.1. Some figures reflecting the size of the two case studies used in our experiments. 
The CPM Plonner was described in chapter 3. The lideo. Innotutor is another groupware application (shown in figure 6.1) that allows a group of people to teview a video that has been previously recorded. End-users can add rextual and graphical annotations to frames of interest. In real time, end-users can see the video playing and the annotarions of each others. All endusers in the session see the video playing, and see each others' annotations.

This chapter reports the results of the CPM Planner and the L'ideo -1nnotutor case studies. These applications are sufficiently large to provide interesting results. They were developed and published in support of other researches, before this project was begun.

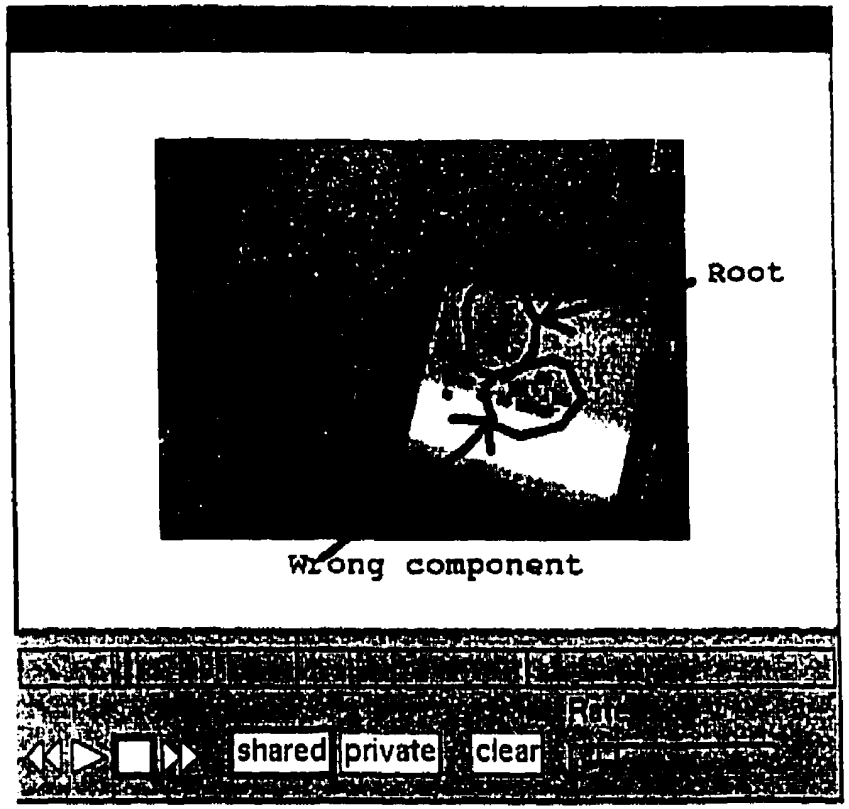

Figure 6.1. The Video Annotator: a collaborative tool for annotating videos (reproduced from [BROWN et al., 1998]). 


\subsection{Conducting the Experiments}

To evaluate . Adligo's generated links, we should compare them to the set of manually generated links, which we trust to be complete and correct. We manually derived the links for the C.P.M Planner and the I'ideo. - Innotator case studies without looking at the links that Aclligo would derive. We then compared our human set of links to those semi-automatically genemed b: - Llligo, and in cases of discrepancy, determined whether the manual or generated links were correct. Those links that had been missed in the manual process were added to the manual set. Through this process, we are confident that we have reached a definitive set of links that can be reated as correct.

Before conducting the experiments, we needed to:

- prepare the L'AN descriptions for our case studies. The LAN descriptions are manually formatted in a text file because we currently do not have a $L: I N$ editor. This file is the task model input to . - delligo. The grammar of this text format is provided in appendix $E$.

- prepare the Clock architecture files. These files are easy to get as we use the C\%iklf:ork:s tool, which is capable of writing the entre architecture in a text file. This file is the architectural model input to Adlito. The yrammar of this text format is provided in appendix F.

- write the application-specific dictionary for each case study. This dictionary is constructed by incrementally adding rules to it. This allows us to see the impact of each added rule on Adligo's performance. 


\begin{tabular}{|c|c|c|c|c|}
\hline \#ofRules & \#ofLinks & CORBECI & MISSING & EXTRA \\
\hline 0 & 0 & 0 & 108 & 0 \\
\hline 1 & 12 & 12 & 96 & 0 \\
\hline 2 & 34 & 34 & 74 & 0 \\
\hline 3 & 44 & 41 & 67 & 3 \\
\hline 4 & 56 & 53 & 55 & 3 \\
\hline 5 & 81 & 71 & 37 & 10 \\
\hline 6 & 110 & 95 & 13 & 13 \\
\hline 7 & $1110+\cdots$ & $96-8+60$ & 12 & 15 \\
\hline 8 & $112 \ldots$ & 972 & 11 & 15 \\
\hline 9 & $114 \mathrm{r}$ & $99 n, \leqslant,+\infty$ & 9. & 15 \\
\hline 10 & 115,4 & $100+4$ & 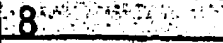 & 15 \\
\hline 11 & $116 \quad-6$ & $101+2$, & $70+r_{0}$ & $15 \ldots$ \\
\hline 12 & $117 \mathrm{~g}+\mathrm{s} s \mathrm{a}$ & $102 x^{2}+4$ & $60,6+$ & 15 \\
\hline$\frac{4}{45}$ & df & $2760=$ & & $\frac{3 f}{a}+4$ \\
\hline
\end{tabular}

Table 6.2. The experiment was run sixteen times in the CPM Planner case study. The links were evaluated by the Analyzer tool.

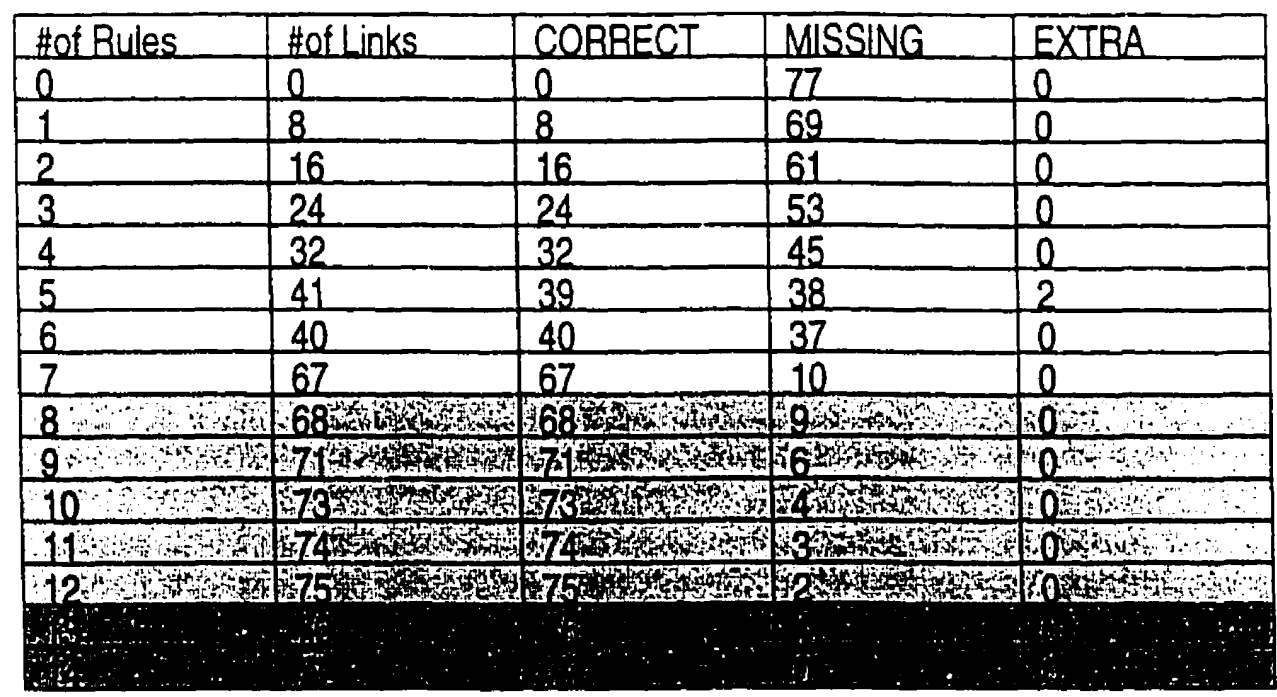

Table 6.3. The experiment was run fifteen times in the Video Annotator case study. The links were evaluated by the Analyzer tool. 
In our case studies, we take advantage of the application-independent dictionaries - standurd and iomponent-based - by adding them to Adligo. Then we incrementally run our experiment, such that in each run we add a new rule to the uppliation-specific dictionary to watch its impact on the performance of the Adligo tool. The experiment stops when we cannot add more rules which improve the quality of the generated links.

Every experiment run involves comparing Adligo's generated links to the human's. The (CPM Planner case study has 108 links generated by hand. We ran the experiment fifteen times because we have fifteen application-specific rules. In each run, we needed to compare the generated links with the manually 108 generated links. We developed an . Incly ier program that accepts the human links and Adligo's in text files, and identifies any missing, extra, and incorrect links. Figures 6.2 and 6.3 summarize the results got from the Analyzer, where we computed their columns as follows:

$$
\begin{aligned}
& \text { CORRECT }=\left\|\mathrm{L}_{4 ;} \cap \mathrm{L}_{11}\right\| \\
& \text { MISSING }=\left\|\mathrm{L}_{-11}-\mathrm{L}_{4}\right\| \\
& \text { EXTRA }=\left\|\mathrm{L}_{\mathrm{C}}-\mathrm{L}_{11}\right\|
\end{aligned}
$$

\subsection{Results}

We rabulate the raw results gathered from the consecutive runs of the Analyar as shown in Tables 6.2 and 6.3 . We use the formulas described in section 6.1 to draw the following graphs: 


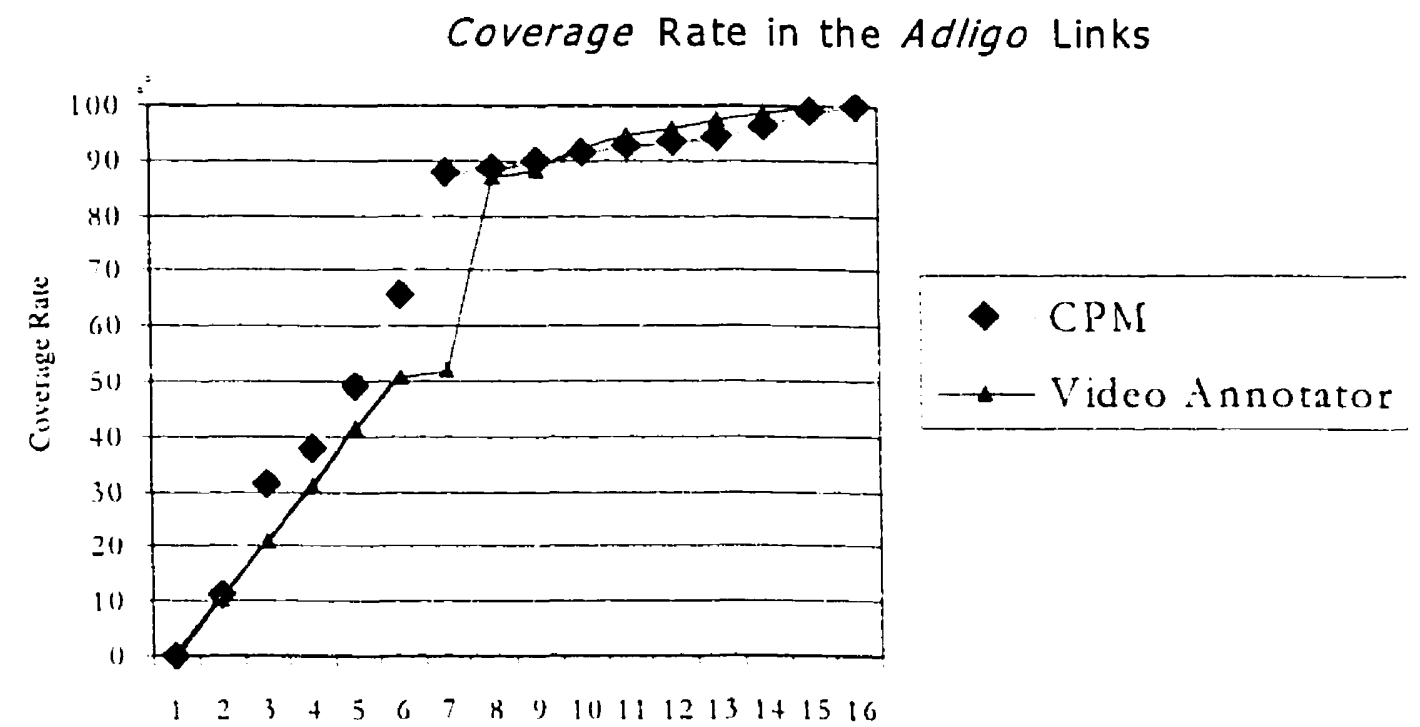

No. of teppliatoon-Speatio Rules

Figure 6.2. Corerage Rate - Inalysis for the . Idligo tool.

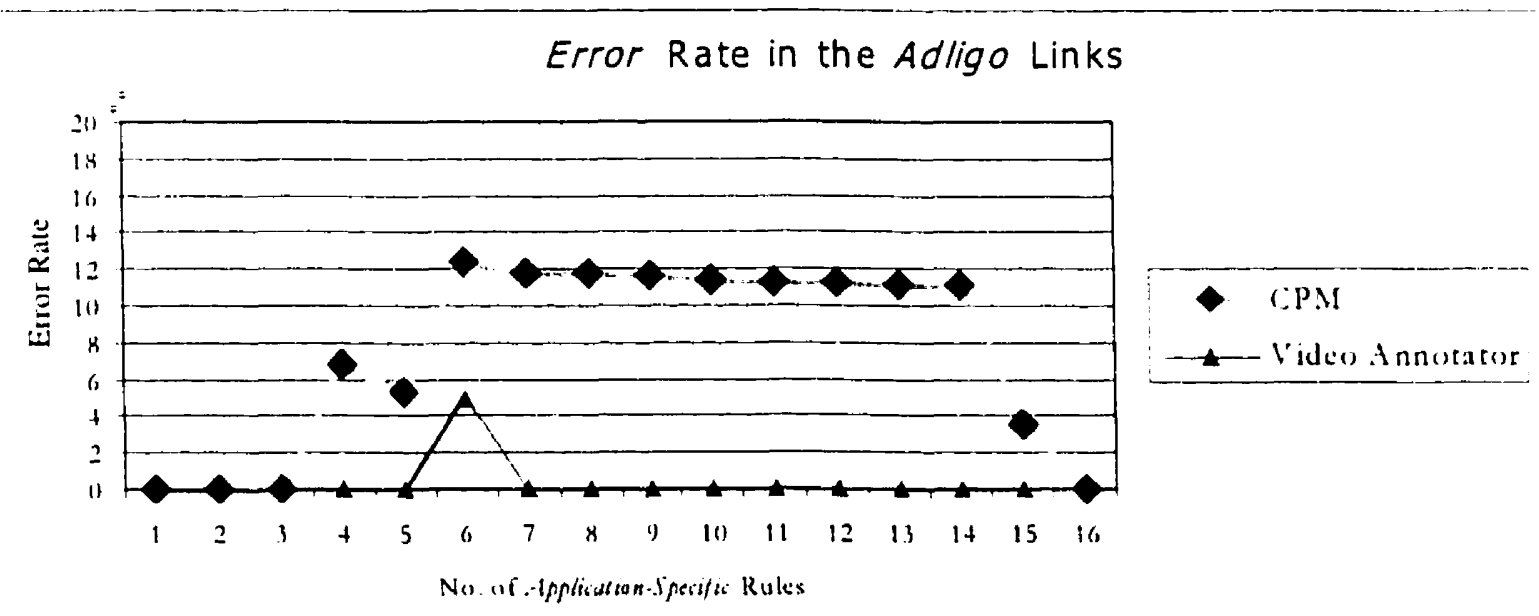

Figure 6.3. Error Rate Analysis for the Adligo tool. 
Figure 6.2 represents the coverage rate for the two case studies. This rate was computed as follows:

$$
\text { Coverage Rate }=\text { CORRECT } /\left\|\mathrm{L}_{11}\right\| \times 100 \%
$$

where CORRECT $=\left\|\mathrm{L}_{i} \cap \mathrm{L}_{11}\right\|$ from Tables 6.1 and 6.2.

Figure 6.3 represents the error rate for the two case studies. This rate was computed as follows:

$$
\text { Error Rate }=\text { EXTR } 1 /\left\|\mathrm{I}_{-} ;\right\| \times 1000^{\circ}{ }_{0}
$$

where EXTR $1=\left\|\mathrm{L}_{9 ;}-\mathrm{L}_{11}\right\|$ from Tables 6.1 and 6.2 .

\subsection{Analysis}

Lsing the chart shown in figure 6.2, we wish to measure the difficulty (i.e., in terms of number of rules the user should add) of getting most of the correct links generated by . Llligo. We observe how many customized rules we need to write to get a high rate of link coverage $\left.(90)^{\prime \prime \prime}\right)$ and to get almost all the correct links $(99 \%)$. We summarize the result in Table 6.3 .

\begin{tabular}{|l|c|c|}
\hline & Link Coverage (90\%) & Link Coverage $(\cong 99 \%)$ \\
\hline CPM Planner & 8 rules & 14 rules \\
\hline Video Annotator & 8 rules & 13 rules \\
\hline
\end{tabular}

Table 6.3. The Diffuculty of getting 90\% and 99\% of Link Coverage by Adligo. 
This table leads to an interesting observation. We could get about $90 \%$ of the links correct by adding the first eight rules in both case studies. If we refer to these rules, we will notice that they are context-related rules. These rules mainly translate the context name used in a L'AN table to a Cluck component. This set of rules represents the first shaded region in Tables 6.2 and 6.3 (to examine these rules, see appendices B and ()). We expect that users will be easily able to identify this set of rules (appendices B and C contain the application-specific dictionaries of both case studies).

To generate most (shown as $99^{\circ}$ ) of the avalable links, we needed to add about extra six rules to the application-specific dictionary. We expect that some of these rules might not be so hard for the user to come up with (the middle region in tables 6.2 and 6.3 ), while we admit that the others are subtle and challenging to write (the bottom region). Adding such rules to reach the boundary of $99 \%$ of correct links is an effort that the Adligo user should decide whether to make.

The chart in figure 6.3 gives a sense of how much Adligo's user can trust its generated links. l.e., the figure shows percentage error of a chosen link (browsed in I'ista for example) mapping the viewer to the wrong part in the opposite model. The graph shows that as we add rules to Adligo, we might encounter a low error rate $(0 \%, 0 \sim 12 \%)$. These errors can be eliminated (as shown in figure 6.3) by adding more rules. Again, adding such subtle rules might not be straightforward for the Adligo user to come up with. 


\subsection{Summary}

This chapter was mainly concerned with evaluating Adligo's performance. We described the methods we used to measure that performance and the nature of experiments we conducted. We also provided the raw results collected from these experiments, and a graphical analysis of them.

From the raw results and their analysis, we notice that the context-reluted rules (the top region in Tables 6.2 and 6.3 ) have a considerable effect on Adligo's performance. The experiments show that by adding these rules alone we could obtain approximately $90 \%$ of the available correct links, with error rates of between 0 "' and $12^{\prime \prime} \%$.

The results gathered from these experiments make us argue that we can tweak Adligo as much as we like to get an extremely high quality of results. In both of case studies, we were able to obtain $100 \%$ of the correct links with an error rate of $0 \%$. Some of the rules required were subtle, however, requiring an expert level of Adligo usage.

L'sers must trade off the burden of adding such extra rules versus the desire for getting extremely accurate results. Such a decision is left to the users of the tool.

Finally, we re-state that further experiments are required to determine whether these results generalize to additional applications of Adligo 
Chapter 7

\section{CONCLUSION}

In this thesis, we have argued that task and architecture models can be semi-automatically: linked. To support this hypothesis, we have developed a computer-based tool, -1dligo, which generates links berween the L'AN as a task model and the Clock architecture as an archirecture model.

Human interrention is still needed in order to enable Adligo to generate sufficiently correct links between the two models. That human assistance was given through the new artifact which we created, called the dittionary. The dictionary has a capability beyond the traditional translation process because it has translation miles. Adligo users write these rules in a simple, pattem langutge specially designed to assist Adligo in finding a LAN description and linking it to its counterpart handler in the Clock architecture.

\section{IE' Still Need More Experiments and Case Studies}

We conducted a few experiments in order to get a sense of Adligo's performance. Our results show that in two case studies, we were able to generate $90 \%$ of possible links with error rate of $0 \%$ to $12 \%$ with only 8 rules in the dictionary. However, we believe that further experiments are required to determine whether these results generalize to all applications of Adligo. (see chapter 6). 
-1pplicubility to Other Types of Models

Although we used the UAN notation to represent task models and the Clock architecrure to represent architectural models, we could use other styles of models to demonstrate the possibility of achieving this thesis' hypothesis. For instance, we expect that if we adopted the ConcurTaskTrees model (see section 2.1.2), it would be easier to handle than the LAN due to its tighter formality. Chapter 2 highlighted some of the common characteristics berween some models. However, we cannor generalize the applicability of our methodology to other models until we try them out.

\section{- lalligo is Helpfint}

We believe that this semi-automatic approach is helpful, especially when our case studies tall in the large size application domain. If we intend to manually generate the links for such case studies, we might need to derive thousands of links in order to map one model to the other. This is a daunting job without computer assistance.

\section{-Ire Designers Going to Communicate Better?}

We intend by this effort to enhance the communication between the different groups of designers involved in developing user interfaces. We gain a sense of this process when we graphically browse the links using the Vista tool (see chapter 3: Design Artifacts Browsed in Vista). We anticipate that this will lead to communication improvement between user interface designers and software engineers, but we need experiments to be able to assert this claim (see section 7.2: Future Work). 
-1t What Time in the Derelopment Process Can We Use Adligo?

It is noteworthy that the linkage process between the models can be carried out at any stage in the development cycle; none of the design artifacts has even to be complete. This is expected to support coevolutionary software development.

\section{Reduing Design Inonsistencies l sing - Adligo}

Is we were experimenting with some case studies described in chapter 6, we noticed that deriving these links might unveil flaws in the design artifacts. This process may discorer that some tasks in the task model have not been implemented, or that some architecrural components are not described in the corresponding task model. In this way, one design model would not be left behind the others, and undesirable inconsistencies can be discovered at any stage during development as the models evolve.

\section{Identifying the Hotspots in the Design, Irtifacts}

As we graphically browse the generated links in Vista, the intensity of the links might lead us risually to the architectural components that are crucial because they implement most of the end-users' tasks. That link intensity might also reveal $L A N$ tasks that are difficult to implement.

\section{Adligo Can Be Tweaked}

Our experiments described in chapter 6 have shown that Adligo can be tuned as much as we wish to get the desired degree of accuracy. That is possible due to the presence of the dictionary rules, which are written in the pattem language. Users can tweak Adligo by even writing rules that explicitly specify links between the UAN and the Clock architecture model. 
- 1bstration of Implementation Details

Since we were ultimately concerned with software architecture, our approach in this research was independent of any programming language details. We have been dealing with architecture handlers, i.e., interface specifications (methods inside components), regardless of which language is used to implement these interfaces. Dealing with the architectural model keeps us at a high level in the design that is independent of any implementation details. For example, the architectures of the applications exposed in this thesis were implemented using a functional language similar to Haskell [HLDAK .IND W.ADLER, 1991] called Clock [GR.HHAM, 1995]. Recently, this language has been replaced by Java in the TCD tool kit [ANDERSON, i999)] which uses the Clock architecture style. Adopting a particular architecture styk, howerer, is still an implementation decision.

\subsection{Future Work}

Inherendy, the user interface design artifacts incrementally evolve as a response to changes in the system [BROWN et al., 1998]. We are interested in investigating design artifacts as they change; some new links may be required, existing links may become invalid. We wish to observe the impact of the evolutionary design on Adligo's performance.

Adligo might incrementally improve its performance in response to evolutionary design by taking advantage of information obtained from one stage to enhance the quality of the links in the other. 


\section{BIBLIOGRAPHY}

[ANDERSON, 1999] G. ANDERSON. Drayonfly: . In implementation-I sut - Irchitecture for Symihronous Gmupware. Master's thesis, Queen's Universiry, Kingston, ON, Canada, 1999.

[BERRY et al., 1997] R. BERRY, S. ISENSEE AND D. ROBERTS. OVID: Obiect View and Interaction Design, Tutorist notes for $\mathrm{CH} H 197,1997$.

[BROWN et al., 1998] J. BROWN, T.C.N. GR.IHAM AND T. WRIGHT. The IISTA Environment for the Coevolutionary Design of User Interfaces. In STCiCHI\% Proceedings. SIGCHI, pp. 376-383, 1998.

[CAlvary et al., 1997] G. Calvary, J. Col"iaz and L. Nicily. From Single-L'ser Architectural Design to PAC*: Generic Software Architecture Model for CSCW. In Procedings of Humun-Futors in Computing Systems CHII 97 (CSA), pages 2+2-24), AC.MI Press Addison-Wesley, 1997.

[C.ARD et al, 1983] S. K. CARD, T. P. MORAN, and A. NEWELL. The Psyibology of HumumComputer Intersition. Hillsadale, NJ: Lawrence Erlbaum, (1983).

[COL"l:AZ, 1997] J. COL"LAZ. PAC-ing the Architecture of Your User Interface. In Proied dings of DSL'-IS'97, Fourth Earographics Workshop on Design, Specification and l'erification of Interactive Systems, pages 15-32, Springer Verlag, 1997.

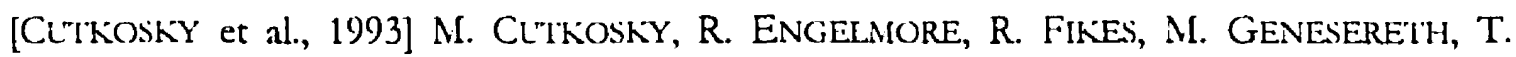
GrLber, W. MARK, J. TENEbAl'M AND J. Weber. PACT: An Experiment in 
Intergrating Concurrent Engineering Systems. In IEEE Computer, 26(1), pages 28 $37,1993$.

[DitPER, 1989] D. DIAPER. Task Observation for Human-Computer Interaction. In Diaper, D., editor, Task. - Inalysis for Human-Computer Interaition. Ellis Honwood, 1989.

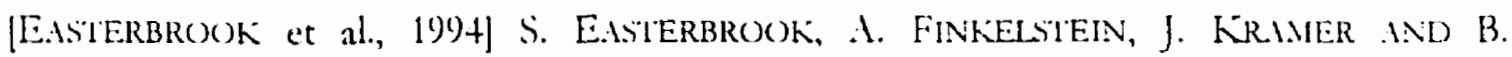
Ne'selbeH. Coordinating Distributed ViewPoints: The Inatomy of a Consistency Check. In International Journal on Concurrent Engineering: Researts and . Ipplications, 2, 3, pp. $209-222,1994$.

[E(KSTElN et al., 1998] K. ECKGTEIN, M. LOY AND D. Wo()D. Jam Sining. ()'Relly and Associates, 1998. ISBN 1-56952-455-X.

[FINKELSTEIN et al., 1992] A. FINKELSIEIN, J. KRTMER, B. NLSEIBEH, L. FINKELSIEIN IND M. GOEDICKE. ViewPoints: A framework for integrating multiple perspectives in system development. In Intermational Joumal of Softwan Engintering and Knonvledge Engineering, 2(1), 31-57, 1992.

[GRAHAM, 1995] T.C.N. GR.HHAM. Detlarative Development of Interative Systems. Volume 2+3 of Berichte der GMD. R. Oldenbourg Verlag, July 1995.

[GRAHAM, 1996] T.C.N. GrthtM. A Method for the Formal Testing of Program Visualization Tools. In Proceedings of the Fourtb Workstsop on Progrum Comprehension, IEEE Computer Society Press, Berlin, Germany, pp. 45-54, March 1996. 
[GRtH.M, 1997] T.C.N. GRtH.M. GroupScape: Integtating Synchronous Groupware and the World Wide Web. In Proceedings of Human-Computer Intersition (INTER-1CT'g7), Chapman and Hall, Sydney, Australia, pp. 547-554, July 1997.

[GRIHAM AND L'RNES, 1996] T.C.N. GRAHAM AND T. LRNES. Linguistic Support for the Evoluaonary Design of Sothware Architectures. In Procedings of the lightienth International Confenente on Sofinan Engineering. IEEE Computer Society Press, Berlin. Germany, pp. +18-427, March 1996.

[GRAHAM AND [IRNES, 1997] T.C.N. GRHHAM AND T. URNES. Integrating Support for Temporal Media into An Architecture for Graphical Lser Interfaces. In Protedinger of ICSE: 97, AC.M Press, pp. 172-183, 1997.

[GRIHAM et al., 1996a] T.C.N. GRMHAM, C.A. MORTON AND T. LRNES. ClockW'orks: Visual Programming of Component-Based Software Architectures. Journal of l'iaul I_anguzuges and Computing, July 1996. Academic Press, pp. 175-196, July 1996.

[GRIH.AM et al, 1996b] T.C.N. GRAHAII, H. D.MKER, C. MORTON, E. TELI:ORI) and T. URNEs. The Cloik Metbodology: Bridging the Gap Between Liser Interface Destinn and Implementation. Technical Report CS-96-()4. York University, August 1996.

[HARTSON AND GRAY, 1990] H. R. HARTSON AND P. GRAY. Temporal Aspects of Tasks in User Action Notations. In Human-Computer Interation, 7(1):1-45, 1990. 
[HARTSON et al. 1990] H. HARTSON, A. SIOCHI AND D. HIX. The LAN: A user-oriented representation for direct manipulation interface designs.. CMI Trunsctions on Information Systems, 8(3):181-203, July 1990.

[HILL, 1992] R.D. HILL. The Abstraction-Link-View paradigm: L'sing constraints to connect user interfaces to applications. In Human fiators in Computing Systems: (.HI ')? Confenence Procedings (Alonterey, CA, L'SA, May 3-7), pages 335-3+2. ACMI Press, 1992.

[HILL et al, 1994] R. D. Hill, T. BRINCK, S.L. R(OHALL, J. F. P.ITTERSON, AND W'. W'L.NER. The Renderyous language and architecture for constructing muld-user applications. KC.W Tranications on Computer.Human Interation, 1(2):81-125, June (199-t).

[HLDAK IND WADLER, 1991] P. HLDAK AND [P. WADLER. Report on the funtional prouramming lenguage Haskell (11.1). Technical Report YALEU/DCS/RR777, Yale L'niversity. August 1901.

UOHNSON et al., 1988] P. JOHNSON, H. JOHNSON, R. WADDINGTON, and A. SHOLLS. Task Related Knowledge Structures: Analysis, modeling and applications. In D. M. Jones \& R. Winder (eds.), People and Computers: From Research to Implementation. Cambridge, U.K.: Cambridge University Press, pp. 35-62, (1988).

[JOHNSON et al., 1993] P. JOHNSON, S. WILSON, P. MLIRKOPOLLOS AND J. PYCOCK. ADEPTadvanced design environment for prototyping with task models. In INTERCHI'७3 
Proceedings of Human Fuctors in Computing, Amsterdam. Reading, MLA: AddisonWesley/ACM Press, p. 56, 1993.

JOHNSON et al., 1995] P. JOHNSON, H. JOHNSON AND S. WILSON. Rapid Prototyping of L ser Interjaces Drien by Task Models in Sienario-Based Design: Emrioning Work and Teithology in Syitem Detropment. John W'ley \& Sons Inc., 1995.

[RRISNER AND POPE, 1988] G.E. KR.tSNER AND S.T. POPE. A cookbook for using the Model-View-Controller user interface paradigm in Smalltalk-80. Journat of (Object. Oriented Progrumming.. 1(3):26-49, August/September 1988.

[MYYER, 1990] B. MYERS. A New Input Model for Handling Input. . KC.W Tramiations on Information System, pages 289-32(). Vol.8, No.3, July 1990).

[MIYERS, 1991] B. MYYER.S. Separating application code from toolkits: Eliminating the Spaghetti of Call-Backs. In Proceedings of the Fourth. - Innual Symposium on L'ser Interfiate Sofivesn and Teimology (LIST '91, Hilton Head, SC, LSA, Nov. 11-13), pages 211-220. ACM Press, 1991.

[NLSEIBEH AND FINKELSTEIN, 1992] B. NLSEIBEH AND A. FinkELSiEIN. ViewPoints: 1 Vehicle for Method and Tool Integration: In Procedings of the IEEE Intermational Workshop on Computer-Alided Software Engineering (C-1SE-92), Montreal, Canada, 6-10 ${ }^{\text {th }}$ July 1992. 
[PAterno et al., 1997a] F. PAterno, C. MLANCINi, and S. MEniCONi. Engineering Task Models. In Procedings of IEEE Conf. Eng. Complex Systems, pp. 69-76, IEEE CS Press, Seprember 1997.

[P.tTERNO et al., 1997b] F. PATERNO, C. MANCINi, and S. MENICONI. ConcurTaskTrees: A Diagrammatic Notation for Specifyng l'ask Models, In Procedding of Inter-. lit's? 362-369, Sydney: Chapman\& Hall, 1997.

[PF:1F: et al., 1985] G. E. Pl:.11Y et al. In D. M. Jones \& R. Winder (eds.), People and Cumputirs: Frnm Restunts to Implementution. Cambridge, U.K.: Cambridge Lniversity Press, pp. $35-62,(1988)$

[PALIN(QLE et al., 1995] P. PALANQLE, R. B.LITDE .AND V. SENGES. Task Model - Syistem Model: Towards an Unifying Formalism. In Proceedings of Humun-Computer Interation, Tokỵo, Japan, July 9-14, 1995.

[SIOCHI AND HARTSON, 1989] A. C. SIOCHI, AND H. R. HARTSON Task-oriented Representation of Asynchronous User Interfaces. In Procedings of CHI'S9 Contentme on Human Fattors in Computing Systems (Austin, Tex, April 30-May 4, 1989). ACM, New York, 1989, 183-188.

[TELIOORD, 1996] E. TELIOORD. Developing a UAN browser in ClockWorks: A Case Simdy of Incremental Development Using the Clock Methodology. Technical Report CS-96-03. York University, June 1996. 
[WILson et al., 1992] S. WILSON, P. MLARkOPOL'LOS, J. PyCOCK AND P. JOHNSON. Modeling Perspectives in User Interface Design. In Proceedings of Eust-IVest Conference on HumunComputer Interaction, pp. 210-216, 1992.

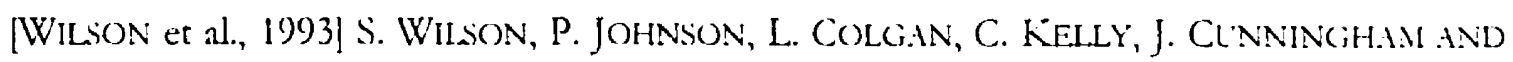
P. MARKopotios. Beyond Hacking: A Model-Based Approach to L ser Interface Design. In J. L. Aly, D. Diaper and S. Guest (eds.), People and Computers I'III. Proiecdings of HCI'93, Loughborough. Cambridge, L.K.: Cambridge Liniversity Press, pp. 215-231, 1993. 


\section{APPENDIX A}

\section{THE STANDARD DICTIONARY}

This standard dictionary is generic and can be used by any Adligo application.

\begin{tabular}{|c|c|c|c|}
\hline & DICTIONARY: Standar & & \\
\hline $\begin{array}{l}\text { Rule } \\
\#\end{array}$ & RULE SCOPE & UAN PATTERN & CLOCK ARCHITECTURE PATTERN \\
\hline 1 & & $M v^{n}$ & mouseButton \\
\hline 2 & & $\mathrm{Mv}$ & mouseButton \\
\hline 3 & & $M^{\mu}$ & mouseButton \\
\hline 4 & & $\sim \$($ obj $)]$ & enter \\
\hline 5 & & {$[\$($ obj $)] \sim$} & leave \\
\hline 6 & & $\sim \$($ obj 1$), \$(o b j 2)]$ & motion I relMotion \\
\hline 7 & & $\sim(\$(o b j 1), \$(o b j 2))$ & motion I relMotion \\
\hline 8 & & $K \$(o b j))^{\prime}$ & key \\
\hline 9 & & $K(\$(o b j))$ & key \\
\hline 10 & & K_UP & arrowkey \\
\hline 11 & & K_DN & arrowkey \\
\hline 12 & & K_RT & arrowkey \\
\hline 13 & & K_LF & arrowkey \\
\hline 14 & & K_TAB & editkey \\
\hline 15 & & K_BS & editkey \\
\hline 16 & & K_DEL & editkey \\
\hline 17 & & K_ESC & editkey \\
\hline 18 & & K_RET & editkey \\
\hline 19 & & $K \_F \$(o b j)$ & functionKey \\
\hline 20 & & $\$($ obj $)++$ & increment $\$(o b j) \mid$ inc $\$(o b j) \mid$ increment \\
\hline 21 & & $\$(o b j)+=1$ & increment $\$(o b j) \mid$ inc $\$(o b j)$ | increment \\
\hline 22 & & lock & lock \\
\hline 23 & & unlock & lock \\
\hline 24 & & unlocked & locked \\
\hline 25 & & have lock & locked \\
\hline 26 & & currentPos & mousePosition \\
\hline 27 & & $(x, y)$ & nodePosition I userPosition \\
\hline 28 & & $\$($ any Var) $:=$ & set\$(any Var) \\
\hline 29 & & $(\$(o b j 1), \$(o b j 2)):=$ & setUserPosition \\
\hline
\end{tabular}




\section{APPENDIX B}

\section{THE CPM PLANNER DICTIONARY}

This is the application-specific dictionary used in the CPM Planner case study. We could obtain $90 \%$ of the possible links by adding the first 8 rules.

\begin{tabular}{|c|c|c|c|}
\hline \multirow[b]{2}{*}{$\begin{array}{l}\text { Rule } \\
\text { \# }\end{array}$} & \multicolumn{3}{|c|}{ DICTIONARY: The CPM Planner } \\
\hline & RULE SCOPE & UAN PATTERN & CLOCK ARCHITECTURE PATTERN \\
\hline 1 & Create a new node & select mode & Network \\
\hline 2 & & mode & button \\
\hline 3 & & $n 1$ & nodeview->connectNode \\
\hline 4 & & $\mathrm{n} 2$ & nodeview->connectNode \\
\hline 5 & & n3 & nodeView->connectNode \\
\hline 6 & $\begin{array}{l}\text { Reposition a } \\
\text { node }\end{array}$ & $n$ & nodeView->browseNode \\
\hline 7 & & nodecountert=1 & incrementcount \\
\hline 8 & & nodeCounter & count \\
\hline 9 & & nodePos $(n):=$ & setNodePosition \\
\hline 10 & & currentmode: $=$ & setmode \\
\hline 11 & & mode & Mode \\
\hline 12 & & $\begin{array}{l}\text { connect } \$(o b j 1) \\
\text { to } \$(o b j 2)\end{array}$ & setConnectionTarget \\
\hline 13 & & lines & edge.View \\
\hline 14 & & rubber-band & rubberBand.view \\
\hline 15 & & $\begin{array}{l}\text { solid line from } \\
\text { n1 to } \mathrm{n} 3\end{array}$ & edge.lineFrom \\
\hline
\end{tabular}




\section{APPENDIX C \\ THE VIDEO ANNOTATOR DICTIONARY}

This is the application-specific dictionary used in the Video Annotator case study. We could obtain $90 \%$ of the possible links by adding the first 8 rules.

\begin{tabular}{|c|c|c|c|}
\hline \multirow[b]{2}{*}{$\begin{array}{l}\text { Rule } \\
\#\end{array}$} & \multicolumn{3}{|c|}{ DICTIONARY: The Video Annotator } \\
\hline & $\begin{array}{l}\text { RULE } \\
\text { SCOPE }\end{array}$ & UAN PATTERN & CLOCK ARCHITECTURE PATTERN \\
\hline 1 & & stop_button & $\begin{array}{l}\text { button->highlightedButton } \\
\text {->buttons->videoButtons }\end{array}$ \\
\hline 2 & & fwd_button & $\begin{array}{l}\text { button->highlightedButton } \\
\text {->buttons->videoButtons }\end{array}$ \\
\hline 3 & & rev_button & $\begin{array}{l}\text { button->highlightedButton } \\
\text {->buttons->videoButtons }\end{array}$ \\
\hline 4 & & ff_button & $\begin{array}{l}\text { button->highlightedButton } \\
\text {->buttons->videoButtons }\end{array}$ \\
\hline 5 & & annotation_mark & indicator \\
\hline 6 & & display frame & video \\
\hline 7 & & drawingBoard & whiteBoard \\
\hline 8 & & currentFrame: $=$ & indicator.setcount \\
\hline 9 & & $\begin{array}{l}\text { start \$(verb) ing frame } \\
\text { counter }\end{array}$ & $\$($ verb) Count \\
\hline 10 & & currentText : $=$ & addText \\
\hline 11 & & currentText+= & addText \\
\hline 12 & & add text & addText \\
\hline 13 & & frame (annotation_mark) & position. count \\
\hline
\end{tabular}




\section{APPENDIX D THE PATTERN LANGUAGE GRAMMAR}

This grammar describes the full syntax of the pattern language used to write dictionary rules.

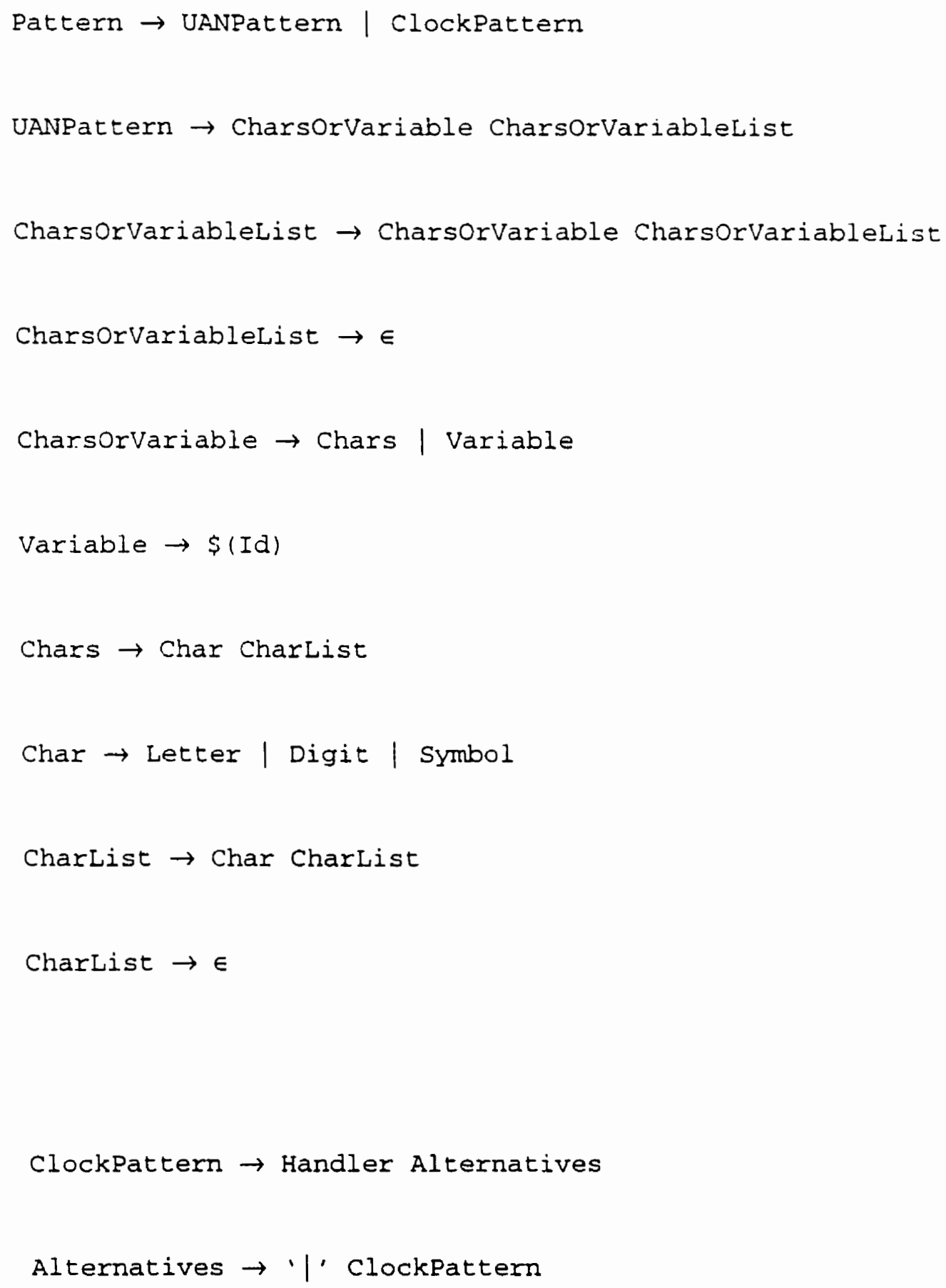




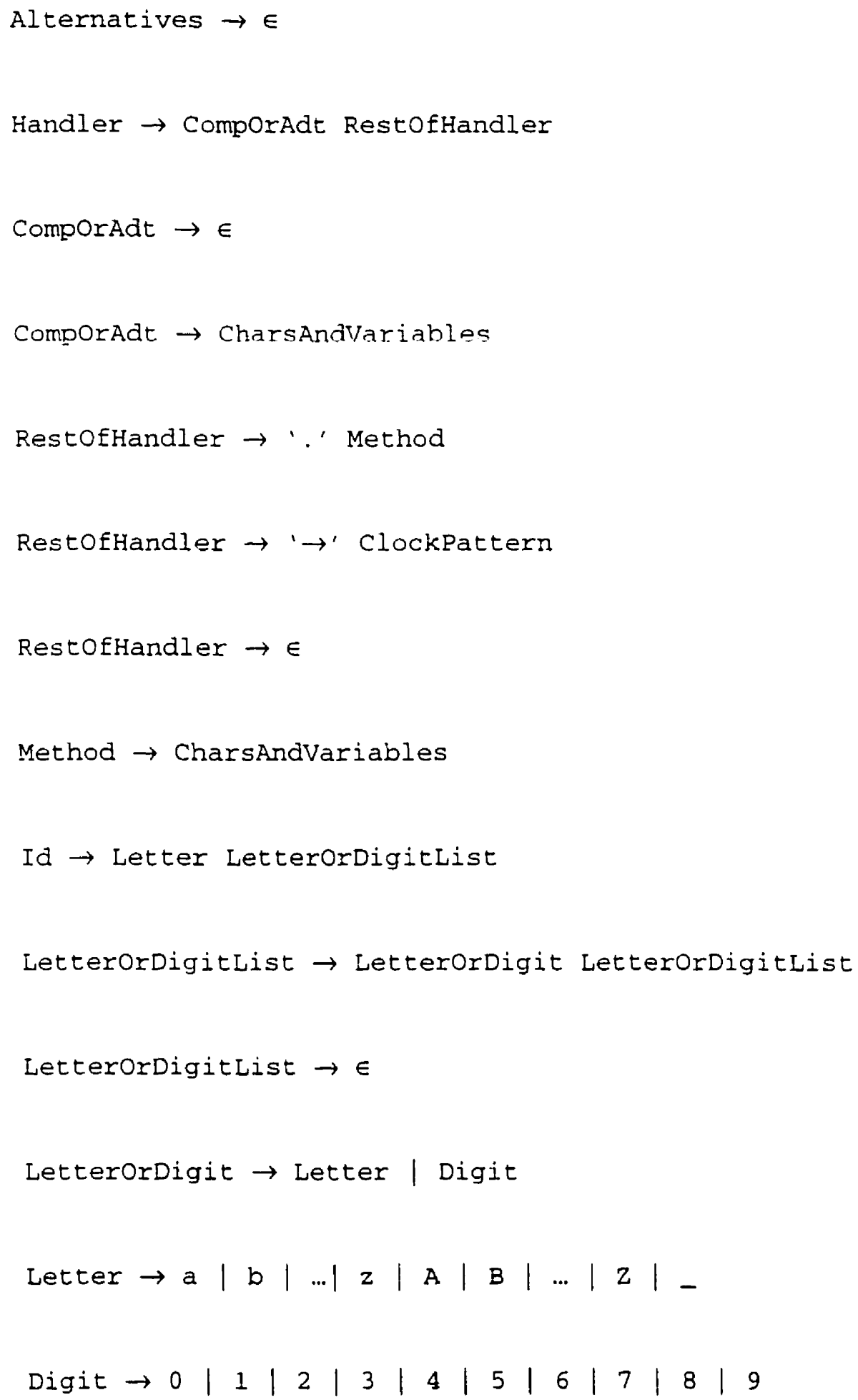


Symbol $\rightarrow$ ! $\mid$ a $|\#| \$|\&| \wedge|\&| *|(\mid)|=1+1-1$ $-1 \cdot 1,1 ? 1 \cdot 1 \cdot 1 \mid 1<1>1 " 1 ; 1: 1 / 1$ ' 


\section{APPENDIX E \\ THE UAN NOTATION GRAMMAR}

This grammar describes the syntax we used to write the UAN notation in a text file to be input to the Adligo tool.

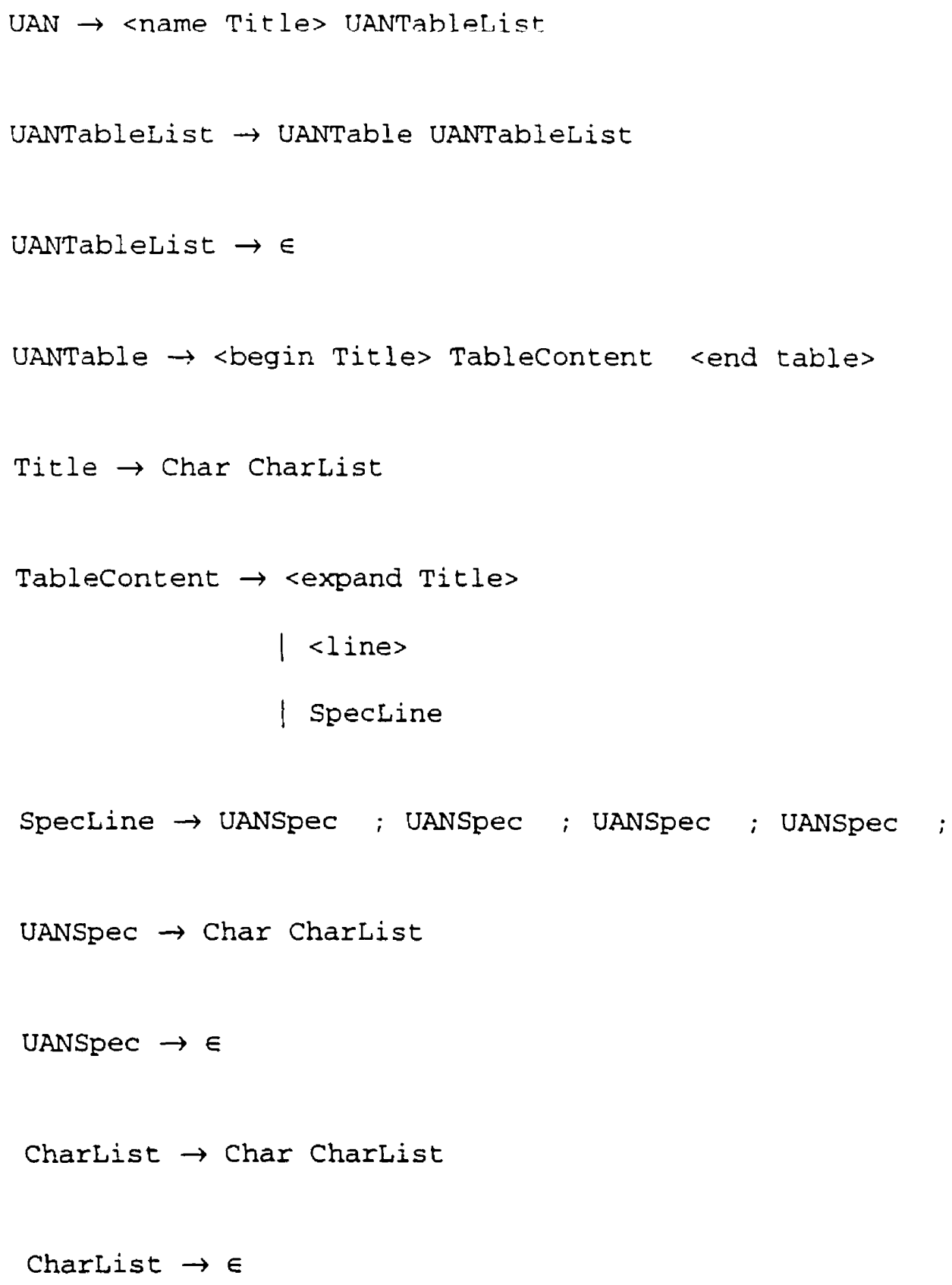


Char $\rightarrow$ Letter | Digit | Symbol

Letter $\rightarrow$ a $\mid$ b $|\ldots| c|c| c|c| c|c| c$

Digit $\rightarrow 0 \mid$\begin{tabular}{l|l|l|l|l|l|l|l|l|l} 
& 1 & 2 & 3 & 4 & 5 & 6 & 7 & 8 & 9
\end{tabular}

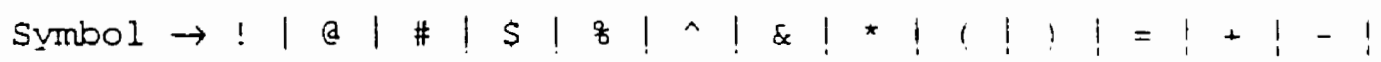

$-1 \cdot 1,1 ?|\cdot| \cdot|1|<1>1,1:|,| \cdot \mid$,

$|[\mid]|\{\mid\} \mid$, 


\section{APPENDIX F \\ THE CLOCK ARCHITECTURE FILE GRAMMAR}

This is the grammar we used to parse the Clock architecture files generated by Clock-Works.

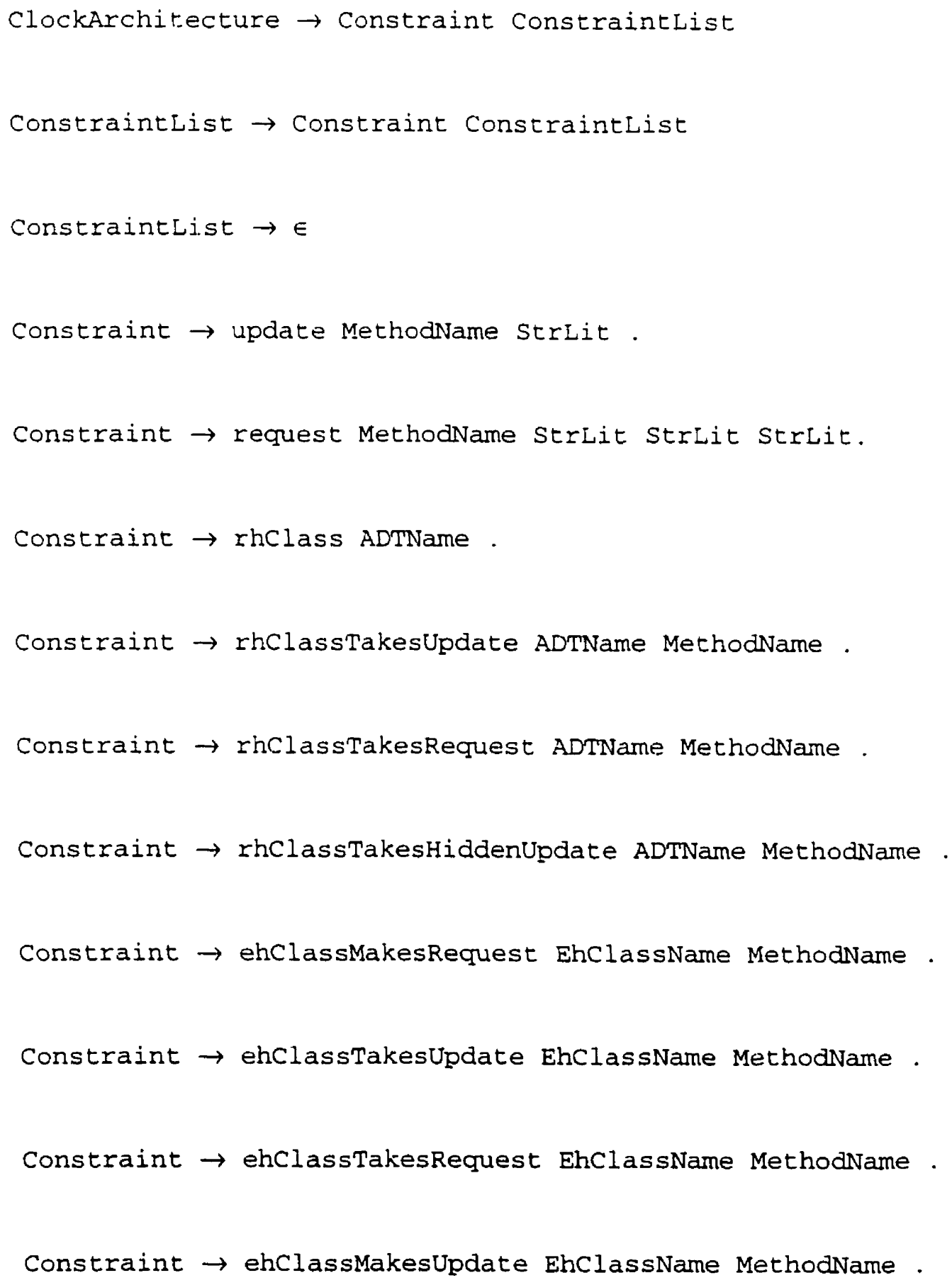




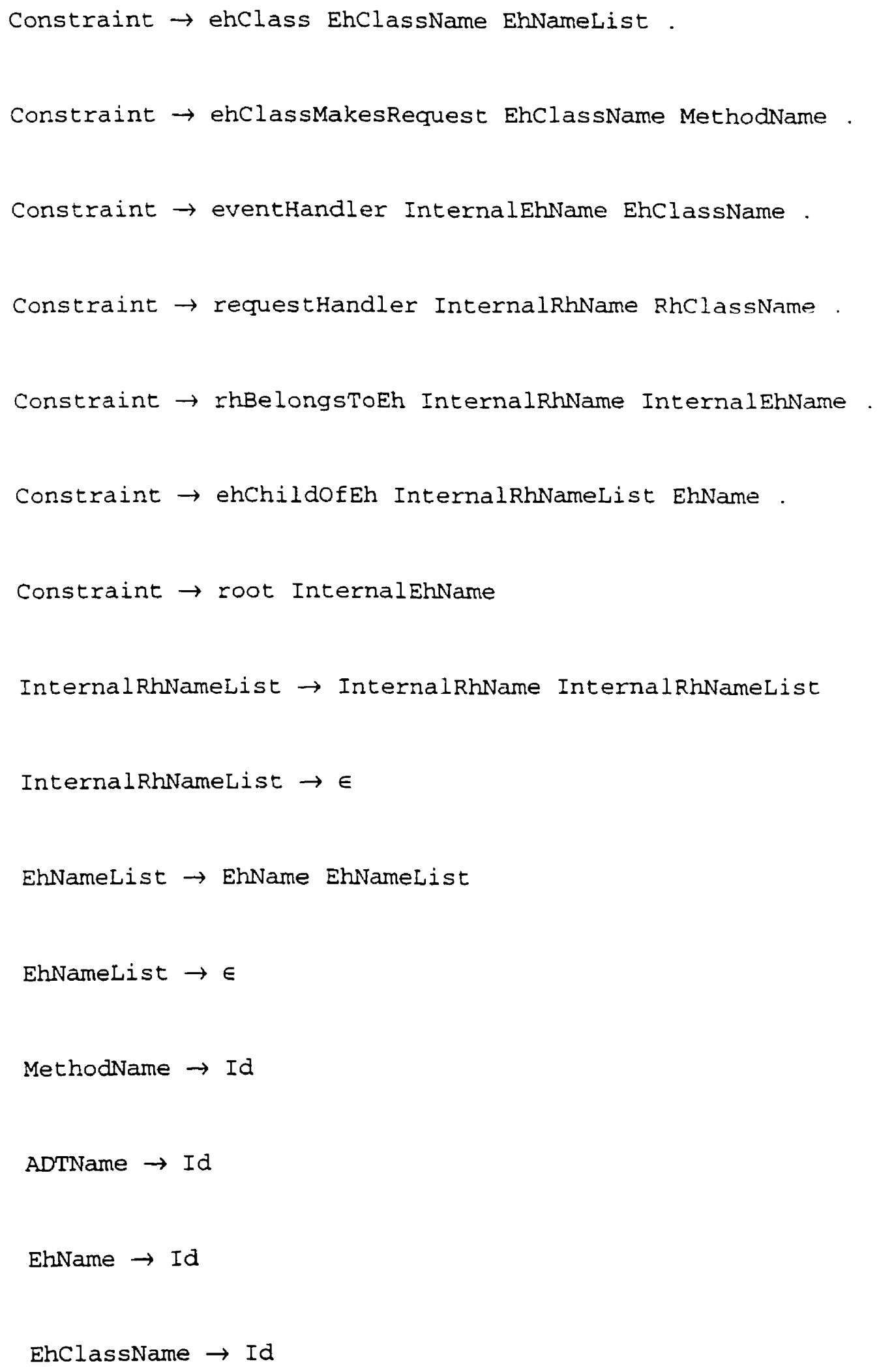




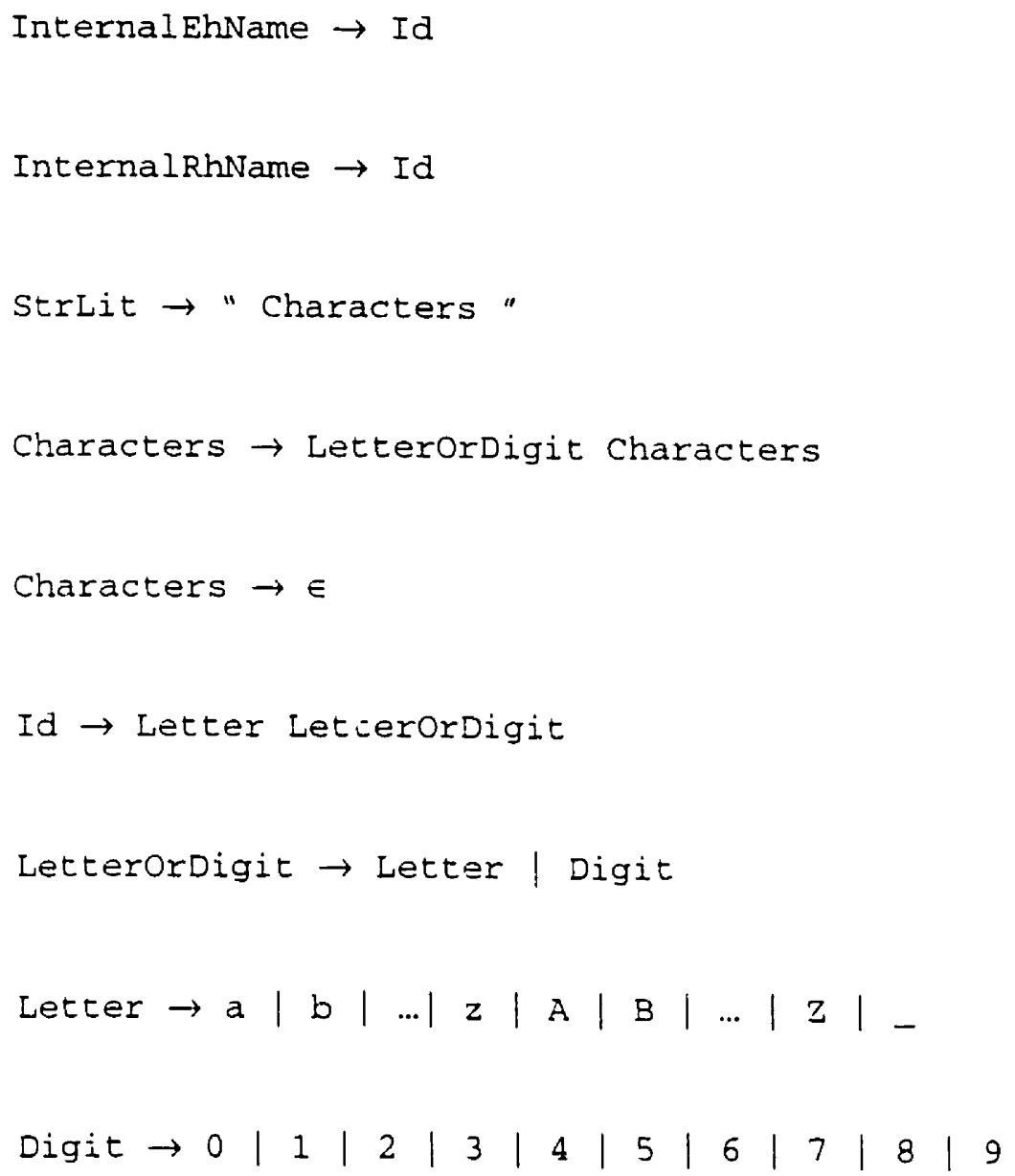

